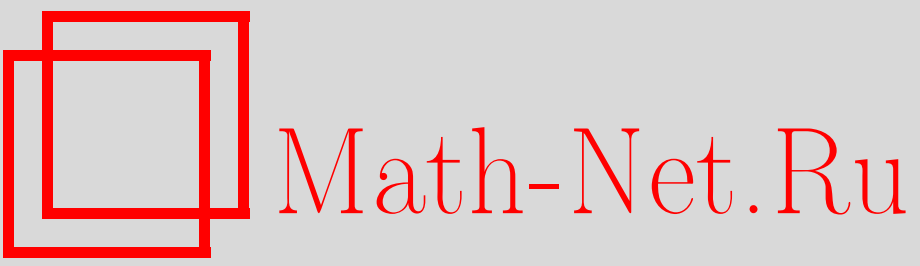

И. А. Тайманов, Секущие абелевых многообразий, тэта-функции и солитонные уравнения, УМН, 1997, том 52, выпуск 1, 149-224

DOI: https://doi.org/10.4213/rm808

Использование Общероссийского математического портала Math-Net.Ru подразумевает, что вы прочитали и согласны с пользовательским соглашением

http://www.mathnet.ru/rus/agreement

Параметры загрузки:

IP: 18.207 .199 .55

26 апреля 2023 г., 13:27:27 


\title{
СЕКУЩИЕ АБЕЛЕВЫХ МНОГООБРАЗИЙ, ТЭТА-ФУНКЦИИ И СОЛИТОННЫЕ УРАВНЕНИЯ
}

\author{
И. А. ТАЙМАНОВ
}

\section{СОДЕРЖАНИЕ}

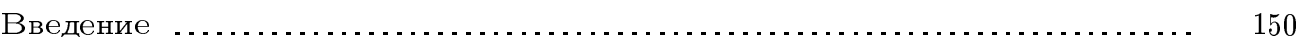

Глава I . Абелевы многообразия и тэта-функции .................. 150

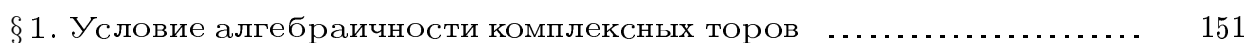

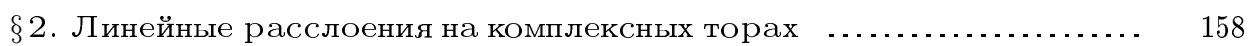

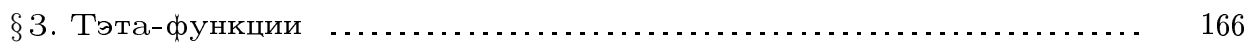

$\S 4$. Тэта-функции и отображения торов в проективные пространства. Секущие абелевых многообразий ..................... 170

Глава II . Тэта-функции римановых поверхностей ................. 176

$\S 5$. Тэта-функции многообразий Якоби .......................... 176

$\S 6$. Тэта-функции многообразий Прима двулистных накрытий с двумя точками ветвления . ................................... 181

$\S 7$. Тэта-ффункции многообразий Прима неразветвленных накрытий 185

Глава III . Многообразия Якоби и солитонные уравнения (проблема Римана-Шоттки, гипотеза Новикова, тройные секущие)

$\S 8$. Функции Бейкера-Ахиезера и кольца коммутирующих дифференциальных операторов ............................... 187

§9. Гипотеза Новикова в проблеме Римана-Шоттки и тройные секущие многообразий Якоби ............................. 193

$\S 10$. Проблема Римана-Шоттки ............................... 199

Глава IV . Многообразия Прима и нелинейные уравнения .......... 206

$\S 11$. Солитонные уравнения и тэта-функции Прима ................. 206

$\S 12$. Методы конечнозонного интегрирования в теории отображения

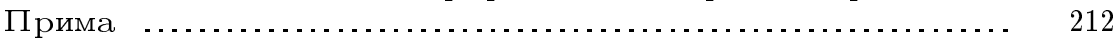

Заключительные замечания $\quad$..................................... 219

Список литературы . . . . . . .

Работа выполнена при финансовой поддержке Российского фонда фундаментальных исследований (грант № 96-01-01889). 


\section{Введение}

Основная цель этого текста - дать краткий обзор применения солитонных уравнений в геометрии абелевых многообразий.

Первым указанием на такую возможность явилась гипотеза Новикова о том, что многообразия Якоби - это, в точности, те главно поляризованные абелевы многообразия, в чьих тэта-функциях интегрируется уравнение Кадомцева-Петвиашвили.

Этой гипотезе предшествовал бурньй начальный этап развития конечнозонного интегрирования солитонных уравнений (Кортевега-де Фриза, цепочки Тода, sin-Gordon и др.) (см. [31], [107]), приведший к появлению метода функций Бейкера-Ахиезера, предложенного Кричевером для построения конечнозонных решений уравнения Кадомцева-Петвиашвили и описания коммутативных колец обыкновенных дифференциальных операторов ранга 1 (см. [61], [62]). Последние результаты и инспирировали гипотезу Новикова. Ее доказательство, полученное Шиотой [98], привело к решению одной из самых старых и знаменитых проблем алгебраической геометрии - проблемы Римана-Шоттки.

В то же время, как показали Арбарелло и де Кончини [2], гипотеза Новикова тесно переплетается с идеями Ганнинга, предложившего описьвать многообразия Якоби как те, которые имеют достаточно большое число тройных секуших (что эквивалентно выполнению тэта-функциональных тождеств специального вида) [44]-[47]. Они опирались на наблюдение Мамфорда о том, что многие солитонные уравнения (Кортевега-де Фриза, Кадомцева-Петвиашвили, sin-Gordon) “скрыты” в тождестве тройной секушей Фэя [82].

В этой статье будет дан обзор как этих работ по гипотезе Римана-Шоттки, так и работ по аналогам гипотезы Новикова для многообразий Прима. Аналогия продолжается дальше, так как уравнения Веселова-Новикова, Ландау-Лифшица, ВКР, интегрируемые в тэта-функциях Прима, также “скрыты” в тождествах четверных секуших Фэя и Бовилля-Дебарра.

Глава I содержит краткое введение в аналитическую теорию тэта-функций (см. также [41], [81]), которое можно считать замкнутым в себе, если добавить информацию о когомологиях с коэффициентами в пучках в объеме, например, книги Чжэня [17].

Глава II содержит необходимую информашию о тэта-функциях Якоби и Прима (подробные доказательства изложены, например, в [36], [41]), а также вьвод формул тройных и четверных секуших.

Главы III и IV посвящены применениям солитонных уравнений к теориям многообразий Якоби и Прима. При этом в $\S 8$ очень кратко изложены необходимые сведения о конечнозонных решениях нелинейных уравнений и функциях Бейкера-Ахиезера. Они широко изложены в обзорах по конечнозонной теории [31], [62], [66], [27] (см. также [8], [64]). Мы надеемся, что этот обзор дополнит их в части, касающейся применения конечнозонной теории к геометрии абелевых многообразий. 


\section{АБЕЛЕВЫ МНОГООБРАЗИЯ И ТЭТА-ФУНКЦИИ}

\section{§1. Условие алгебраичности комплексных торов}

\section{1. Алгебраические многообразия.}

Комплексное многообразие называется проективныцм алгебраическим, если оно вкладывается в комплексное проективное пространство $\mathbb{C P}^{n}$ как множество нулей системы однородных многочленов.

Пространство $\mathbb{C P}^{n}$ определяется как фактор-пространство $\mathbb{C}^{n+1} \backslash\{0\}$ по действию $\mathbb{C}^{*}=\mathbb{C} \backslash\{0\}:$

$$
\left(z^{0}, z^{1}, \ldots, z^{n}\right) \rightarrow\left(\lambda z^{0}, \lambda z^{1}, \ldots, \lambda z^{n}\right), \quad \lambda \in \mathbb{C}^{*} .
$$

На этом пространстве задана метрика Фубини-Штуди, имеющая в однородных координатах $\left(z^{0}: \ldots: z^{n}\right)$ вид

$$
\frac{\left(\sum_{j} z^{j} \bar{z}^{j}\right) \cdot\left(\sum_{k} d z^{k} d \bar{z}^{k}\right)-\left(\sum_{j} z^{j} d \bar{z}^{j}\right) \cdot\left(\sum_{k} \bar{z}^{k} d z^{k}\right)}{\left(\sum_{k} z^{k} \bar{z}^{k}\right)^{2}} .
$$

Этой эрмитовой метрике сопоставим однозначно форму

$$
\omega=\frac{\sqrt{-1}}{2 \pi} \cdot \frac{\left(\sum_{j} z^{j} \bar{z}^{j}\right) \cdot \sum_{k} d z^{k} \wedge d \bar{z}^{k}-\left(\sum_{j} z^{j} d \bar{z}^{j}\right) \wedge\left(\sum_{k} \bar{z}^{k} d z^{k}\right)}{\left(\sum_{k} z^{k} \bar{z}^{k}\right)^{2}} .
$$

Прямьми вычислениями проверяется следующее:

1) форма $\omega$ замкнута;

$2)$ класс когомологий $[\omega]$ - целочисленный: $[\omega] \in H^{2}\left(\mathbb{C P}^{n} ; \mathbb{Z}\right)$.

Комплексные многообразия, обладаюшие указанными выше свойствами, выделяются в отдельные классы:

1) если на компактном комплексном многообразии $M$ существует эрмитова метрика $h_{i j} d z^{i} d \bar{z}^{j}$ такая, что ассоциированная с ней форма

$$
\omega=\frac{\sqrt{-1}}{2 \pi} h_{i j} d z^{i} \wedge d \bar{z}^{j}
$$

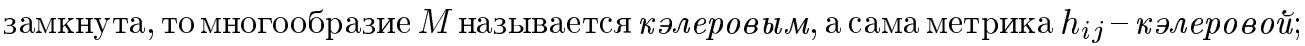

2 ) если на кэлеровом многообразии $M$ форма $\omega$, ассоциированная с кэлеровой метрикой, реализует целочисленный класс когомологий $\left([\omega] \in H^{2}(M ; \mathbb{Z})\right)$, то многообразие $M$ назьвается ходжевым, а сама форма $\omega-$ ходжсевой.

В формуле (1) и в дальнейшем, если не оговорено противное, под повторением верхних и нижних индексов в одном выражении мы подразумеваем суммирование по ним.

Дадим и инвариантное определение формы $\omega$. Это достаточно сделать локально, в касательном пространстве к точке. Разложим эрмитову форму

$$
(v, w)=h_{i j} v^{i} \bar{w}^{j}
$$


в сумму вешественной и мнимой частей:

$$
H(v, w)=H_{R}(v, w)+\sqrt{-1} H_{I}(v, w),
$$

где вешественные формы $H_{R}$ и $H_{I}$ билинейны над $\mathbb{R}$. Из условий эрмитовости следует, что форма $H_{R}$ симметрична и положительно определена, а форма $H_{I}$ кососимметрична. Так как $H(\sqrt{-1} v, w)=\sqrt{-1} H(v, w)$, то

$$
H_{R}(v, w)=H_{I}(\sqrt{-1} v, w) .
$$

Таким образом, вся эрмитова метрика определяется кососимметрической формой $H_{I}$. В каждой точке $x \in M$ положим

$$
\omega(x)(v, w)=-\frac{1}{\pi} H_{I}(x)(v, w),
$$

где $v, w \in T_{x} M$.

Эквивалентность (1) и (4) очевидна для метрик диагонального вида. Обший случай сводится к этому заменой базиса.

\section{2. Теоремы Кодаиры и Чжоу.}

Легко заметить, что свойства кэлеровости и ходжевости наследуются подмногообразиями: если многообразие $Y$ является подмногообразием кэлерова (ходжева) многообразия $X$, то кэлерова метрика (ходжева форма) на $X$ индуцирует при вложении кэлерову метрику (ходжеву форму) на $Y$. Отсюда следует, что проективные алгебраические многообразия являются ходжевыми. Кодаира доказал, что верно и обратное утверждение.

1.2.1. ТЕОРЕМА КОДАИРЫ. Комплексное многообразие проективно алгебраично тогда и только тогда, когда оно ходжево.

Доказательство теоремы Кодаиры состоит в построении вложения ходжева многообразия в проективное пространство достаточно большой размерности и применении следуюшей теоремы Чжоу.

1.2.2. ТЕОрема Чжоу. Аналитическое подмножество $X \subset \mathbb{C P}^{n}$ выделяется в пространстве $\mathbb{C P}^{n}$ как множество нулей системы однородных многочленов.

Для комплексных торов эквивалентность ходжевости и алгебраичности была доказана Лефшшецем (см. §4).

\section{3. Критерий ходжевости комплексных торов.}

Комплексньй тор $\mathbb{C}^{n} / \Lambda$ (где $\Lambda$ - решетка ранга $2 n$ в $\mathbb{C}^{n}$ ) называется абелевым многообразием (или абелевым тором), если он является проективным алгебраическим многообразием. Согласно теореме Леффшеца это эквивалентно ходжевости тора. 
1.3.1. КРИТЕРИЙ РИмАНА. Комплексный тор $M=\mathbb{C}^{n} / \Lambda$ является ходжевым многообразием тогда и только тогда, когда существует комплексныи базис в $\mathbb{C}^{n}$, в котором решетка $\Lambda$ принимает вид

$$
\Lambda=\Delta_{\delta} N_{1}+\Omega N_{2}, \quad N_{1}, N_{2} \in \mathbb{Z}^{n},
$$

где

1) $\Delta_{\delta}-$ диагональная $(n \times n)$-матрица $\operatorname{diag}\left(\delta_{1}, \ldots, \delta_{n}\right)$ с челочисленными коәффициентами, причем $\delta_{j}>0$ и $\delta_{k}$ делит $\delta_{k+1}$ при любом $k=1, \ldots,(n-1)$;

2) матрича $\Omega$ симметрична;

3) матрица $\operatorname{Im} \Omega$ положительно определена.

ДоКАЗАТЕЛЬСТВО КРИТЕРИЯ РИМАНА. Предположим, что комплексньй тор $M=\mathbb{C}^{n} / \Lambda$ ходжев, а именно, на $\mathbb{C}^{n}$ определена эрмитова метрика $h_{i j} d z^{i} d \bar{z}^{j}$ такая, что она инвариантна относительно сдвигов на векторы решетки $\Lambda$ и порождает метрику на $M$, которая определяет на торе ходжеву структуру.

1.3.2. Пусть $x^{1}, \ldots, x^{2 n}$ - линейные $($ по $\mathbb{R})$ координать на $\mathbb{R}^{2 n}=\mathbb{C}^{n} u$ $\omega=\omega_{i j} d x^{i} \wedge d x^{j}-$ замкнутая 2-форма на торе $\mathbb{C}^{n} / \Lambda$. Тогда оператор усреднения

$$
\omega_{i j} \rightarrow \widetilde{\omega}_{i j}=\frac{\int_{\Pi} \omega_{i j}\left(z^{1}, \ldots, z^{n}\right) d z^{1} \wedge \cdots \wedge d z^{n}}{\int_{\Pi} d z^{1} \wedge \cdots \wedge d z^{n}}
$$

(где П - фундаментальная область решетки $\Lambda$ ), переводит $\omega$ в когомологичную ей 2-форму с постоянными коэффициентами. Ходжевы формы переходят при этом в ходжевй.

Доказательство очевидно.

1.3.3. Замкнутая 2-форма $\omega$ на торе $M=\mathbb{C}^{n} / \Lambda$ порождает на решетке $\Lambda$ кососимметрическую 2-форму $Q$. Значение $Q$ на паре векторов $(u, v)$ равно интегралу от $\omega$ по 2-тору, натянутому на векторы и и $v$. Если $[\omega] \in H^{2}(M ; \mathbb{Z})$, то $Q$ челочисленна. Если форма $\omega$ ходжева, то $Q$ невырожденна.

Пусть $v, w \in \Lambda$ и $\Pi_{v, w}$ - параллелограмм, натянутьй на векторы $v$ и $w$ в $\mathbb{C}^{n}$. Он проектируется в 2 -цикл $T_{v, w}$ на торе $M$. Определим форму $Q$ как

$$
Q(v, w)=\int_{T_{v, w}} \omega
$$

Если $\omega$ ходжева, то согласно (1) форма $\omega^{n}$ пропорциональна форме объема и поэтому $Q$ невырожденна. Предложение 1.3 .3 доказано.

1.3.4. КРИТЕРИЙ ФРОБЕНИУСА. Комплексный тор $M=\mathbb{C}^{n} / \Lambda$, где $\Lambda$ - peшетка ранга $2 n$, является ходжевым многообразием тогда и только тогда, когда существует такая вещественная кососимметрическая билинейная (над $\mathbb{R})$ форма $Q$ на $\mathbb{C}^{n} \times \mathbb{C}^{n}$, ито

1) форма $\langle v, w\rangle=Q(\sqrt{-1} v, w)$ симметрична и положительно определена;

2) форма $Q$ принимает иелочисленные значения на $\Lambda \times \Lambda$. 
ДОКАЗАТЕЛЬСТВО КРИТЕРИЯ ФРОБЕНИУСА. Если тор Ходжев, то возьмем на нем эрмитову метрику с постоянными коэффициентами, порождаюшую ходжеву форму. Ассоциированная с ней форма $\omega$ согласно (4) и 1.3.3 удовлетворяет условиям 1) и 2).

Если же существует форма $Q$, удовлетворяющая условиям 1.3.4, то эрмитова метрика $\langle v, w\rangle=\pi(Q(\sqrt{-1} v, w)+\sqrt{-1} Q(v, w))$ порождает ходжеву форму, ассоциированную с ней.

Критерий Фробениуса доказан.

1.3.5. Если на $\Lambda=\mathbb{Z}^{2 n}$ задана челочисленная кососимметрическая невырожденная 2-форма $Q$, то в подходящем базисе $\lambda_{1}, \ldots, \lambda_{2 n}$ она приводится $к$ виду

$$
Q=\left(\begin{array}{cc}
0 & \Delta_{\delta} \\
-\Delta_{\delta} & 0
\end{array}\right)
$$

где $\Delta_{\delta}-$ иелочисленная диагональная матрича $\operatorname{diag}\left(\delta_{1}, \ldots, \delta_{n}\right)$, причем $\delta_{k}>0$ $u \delta_{j}$ делит $\delta_{j+1}$ для любого $j=1, \ldots, n-1$. Набор чисел $\delta_{k}$, удовлетворяющих данным условиям, является инвариантом формы $Q$.

Докажем это предложение.

Рассмотрим всевозможные положительные значения формы $Q\left(\lambda, \lambda^{\prime}\right)$ и выберем среди них минимальное $-\delta_{1}=Q\left(\lambda_{1}, \lambda_{n+1}\right)$. Обозначим через $\Lambda^{\prime}$ ортогональное дополнение в $\Lambda$ к подрешетке $\mathbb{Z}\left\{\lambda_{1}, \lambda_{n+1}\right\}$. Если $\lambda \in \Lambda$, то

$$
\lambda+\frac{Q\left(\lambda, \lambda_{1}\right)}{\delta_{1}} \lambda_{n+1}-\frac{Q\left(\lambda, \lambda_{n+1}\right)}{\delta_{1}} \lambda_{1} \in \Lambda^{\prime}
$$

Отсюда следует, что $\Lambda^{\prime}=\mathbb{Z}^{2 n-2}$ и ограничение формы $Q$ на $\Lambda^{\prime}$ удовлетворяет условиям предложения 1.3.5. Поэтому, применяя последовательно данную процедуру, мы выберем в решетке $\Lambda$ базис $\lambda_{1}, \ldots, \lambda_{2 n}$, по отношению к которому форма $Q$ будет иметь вид (6).

Докажем от противного, что $\delta_{1}$ делит $\delta_{2}$. Пусть $\delta$ - наибольший общий делитель $\delta_{1}$ и $\delta_{2}$ и $\delta<\delta_{1}$. Тогда сушествуют такие целье числа $k$ и $m$, что $\delta=k \delta_{1}+m \delta_{2}$. Но

$$
Q\left(k \lambda_{1}+m \lambda_{2}, \lambda_{n+1}+\lambda_{n+2}\right)=\delta<\delta_{1}
$$

что противоречит выбору $\delta_{1}$. Значит, $\delta_{1}$ делит $\delta_{2}$. Аналогично доказьвается, что $\delta_{j}$ делит $\delta_{j+1}$ для любого $j, 1 \leqslant j \leqslant n-1$.

Из построения последовательности $\left\{\delta_{j}\right\}$ видно, что этот набор чисел является инвариантом формы $Q$. Предложение 1.3.5 доказано.

1.3.6. Пусть $\omega$ - ходжсева форма, ассочиированная с әрмитовой метрикой $c$ постоянными коэффициентами на ходжевом торе $M=\mathbb{C}^{n} / \Lambda, u \lambda_{1}, \ldots, \lambda_{2 n}-$ базис решетки $\Lambda$, в котором форма $Q$ имеет вид (6). Тогда векторы $\lambda_{1}, \ldots, \lambda_{n}$ образуют комплексный базис $\mathbb{C}^{n}$. 
ДОКАЗАТЕЛЬСТВО ПРЕДЛОЖЕНИЯ 1.3.6. Так Как коэффициенты эрмитовой формы постоянны, то форма $Q$, распространенная по линейности (над $\mathbb{R})$ на $\mathbb{C}^{n}$, совпадает c $\omega$ и определяет эрмитову метрику по формулам (2)-(4).

Рассмотрим индуктивно определяемую систему векторов:

$$
v_{1}=\lambda_{1}, v_{2}=\lambda_{2}-\frac{Q\left(\sqrt{-1} \lambda_{2}, v_{1}\right)}{Q\left(\sqrt{-1} v_{1}, v_{1}\right)} v_{1}, \ldots, v_{k}=\lambda_{k}-\sum_{j<k} \frac{Q\left(\sqrt{-1} \lambda_{k}, v_{j}\right)}{Q\left(\sqrt{-1} v_{j}, v_{j}\right)} v_{j}, \ldots
$$

Согласно (3), (4) все выражения $Q\left(\sqrt{-1} \lambda_{k}, v_{j}\right) / Q\left(\sqrt{-1} v_{j}, v_{j}\right)$ вешественны. Из (2) и $(6)$ следует, что $\left(v_{j}, v_{k}\right)=0$ при $j \neq k$. Так как $Q\left(v_{k}, \lambda_{n+k}\right) \neq 0$, все векторы $v_{1}, \ldots, v_{n}$ отличны от нуля и образуют комплексный базис в $\mathbb{C}^{n}$. Так как линейные оболочки над $\mathbb{C}$ систем $\left(v_{1}, \ldots, v_{n}\right)$ и $\left(\lambda_{1}, \ldots, \lambda_{n}\right)$ совпадают, то отсюда следует предложение 1.3.6.

Теперь перейдем непосредственно к доказательству критерия Римана.

Пусть $M=\mathbb{C}^{n} / \Lambda$ - ходжев тор. Согласно предложениям 1.3.2-1.3.6 существуют базис $\mathscr{V}_{0}=\left(\lambda_{1}, \ldots, \lambda_{2 n}\right)$ решетки $\Lambda$ и ходжева форма $\omega$ с постоянными коэффициентами такие, что форма $\omega$ имеет в этом базисе вид

$$
\omega=\sum_{k=1}^{n} \delta_{k} d y^{k} \wedge d y^{n+k}
$$

Выберем в качестве комплексного базиса в $\mathbb{C}^{n}$ следуюшую систему векторов:

$$
\mathscr{V}_{\mathbb{C}}=\left(\frac{1}{\delta_{1}} \lambda_{1}, \ldots, \frac{1}{\delta_{n}} \lambda_{n}\right)
$$

а в качестве вещественного базиса

$$
\mathscr{V}_{\mathbb{R}}=\left(\frac{1}{\delta_{1}} \lambda_{1}, \ldots, \frac{1}{\delta_{n}} \lambda_{n}, \lambda_{n+1}, \ldots, \lambda_{2 n}\right) .
$$

Тогда $\Lambda$ имеет вид (5), а формулы перехода от базиса $\mathscr{V}_{\mathbb{R}}$ к $\mathscr{V}_{\mathbb{C}}-$

$$
\left(\begin{array}{c}
z^{1} \\
\vdots \\
z^{n}
\end{array}\right)=\left(I_{n}, \Omega\right)\left(\begin{array}{c}
x^{1} \\
\vdots \\
x^{2 n}
\end{array}\right),
$$

где $I_{n}$ - единичная $(n \times n)$-матрица.

Из (7)-(9) следует, что форма $\omega$ имеет в базисе (8) вид

$$
\omega=\sum_{k=1}^{n} d x^{k} \wedge d x^{n+k}
$$

Так как $\omega$ ассоциирована с ходжевой метрикой $h_{i j}$ согласно (1), то из (10) следует, что

$$
\omega=\frac{\sqrt{-1}}{2 \pi} h_{i j}\left(d x^{i}+\Omega_{k}^{i} d x^{n+k}\right) \wedge\left(d x^{j}+\Omega_{l}^{j} d x^{n+l}\right)=\sum_{m=1}^{n} d x^{m} \wedge d x^{n+m} .
$$


Уравнение (11) с учетом симметричности формы $h_{i j}$ эквивалентно

$$
\frac{\sqrt{-1}}{2 \pi} h_{k j}\left(\bar{\Omega}_{l}^{j}-\Omega_{l}^{j}\right) d x^{k} \wedge d x^{n+l}=\sum_{m=1}^{n} d x^{m} \wedge d x^{n+m}
$$

и

$$
T_{l m}=T_{m l}, \quad \text { где } T_{l m}=h_{k j} \Omega_{m}^{k} \bar{\Omega}_{l}^{j} .
$$

Но из (12) следует, что

$$
T_{l m}=h_{k j} \Omega_{l}^{k} \Omega_{m}^{j}+\frac{2 \pi}{\sqrt{-1}} \Omega_{l}^{m}
$$

Теперь мы можем заключить, что

1) матрица $\operatorname{Im} \Omega$ положительно определена (это следует из (12));

2) матрища $B$ симметрична: $\Omega_{m}^{l}=\Omega_{l}^{m}$ (это следует из (13) и (14)).

Таким образом, мы доказали, что если тор $\mathbb{C}^{n} / \Lambda$ является ходжевым многообразием, то он имеет вид, указанный в формулировке критерия Римана.

Если же тор имеет такой вид, то из (11)-(14) следует, что эрмитова форма $h_{i j} d z^{i} \wedge d \bar{z}^{j}$, определяемая по (12), является ходжевой, а тор является ходжевым многообразием.

Критерий Римана доказан.

1.4. Поляризации и модули абелевых многообразий.

Мы будем в этой части $§ 1$ отождествлять ходжевы и абелевы торы. Строгое доказательство совпадения этих классов будет дано в $\S 4$.

Ходжев тор (или абелево многообразие) $M=\mathbb{C}^{n} / \Lambda$ называется поляризованныл если на нем фиксирован класс когомологий ходжевой формы $\omega$ на $M$. Этот класс $[\omega] \in$ $H^{2}(M ; \mathbb{Z})$ назьвается поляризацией многообразия $M$.

Согласно 1.3.3 и 1.3.5 поляризация [ $\omega]$ однозначно определяет целочисленную кососимметрическую невырожденную 2-форму

$$
Q: H^{1}(M) \times H^{1}(M) \rightarrow \mathbb{Z}
$$

которая в подходящем базисе решетки $\Lambda$ приводится к виду (6), причем набор положительных целых чисел $\delta_{1}, \ldots, \delta_{n}$ определяется по ней однозначно. Этот набор $\delta_{1}, \ldots, \delta_{n}$ называется типом поляризачии. Абелево многообразие назьвается главно поляризованнылм, если $\delta_{1}=\cdots=\delta_{n}=1$.

Поляризованные абелевы многообразия $\left(M_{1},\left[\omega_{1}\right]\right)$ и $\left(M_{2},\left[\omega_{2}\right]\right)$ назьваются әквивалентными, если сушествует биголоморфное отображение $f: M_{1} \rightarrow M_{2}$ такое, что $f^{*}\left(\left[\omega_{2}\right]\right)=\left[\omega_{1}\right]$. Для заданных пар $(n, \delta)$ классы эквивалентности $n$-мерных абелевых многообразий с поляризацией типа $\delta$ образуют комплексные многообразия $\mathscr{A}_{n}^{\delta}-$ пространства модулей абелевьх многообразий.

Пространства модулей $n$-мерных главно поляризованных абелевых многообразий мы будем обозначать через $\mathscr{A}_{n}$.

Пространства модулей $\mathscr{A}_{n}^{\delta}$ устроены следующим образом. 
Обозначим через $\mathscr{H}_{n}$ верхнюю полуплоскость Зигеля, определяемую как совокупность симметричных $(n \times n)$-матриц с положительно определенньми мнимыми частями. Из доказательства критерия Римана и из предложений 1.3.3 и 1.3.5 следует, что каждое $n$-мерное абелево многообразие с поляризацией типа $\delta$ эквивалентно тору $\mathbb{C}^{n} / \Lambda$, где $\Lambda$ имеет вид (7) и $\Omega \in \mathscr{H}_{n}$. Достаточно очевидно, что два поляризованных абелевых многообразия $M_{1}=\mathbb{C}^{n} /\left\{\Delta_{\delta} \mathbb{Z}^{n}+\Omega_{1} \mathbb{Z}^{n}\right\}$ и $M_{2}=\mathbb{C}^{n} /\left\{\Delta_{\delta} \mathbb{Z}^{n}+\Omega_{2} \mathbb{Z}^{n}\right\}$ эквивалентны тогда и только тогда, когда эти решетки сопряжены преобразованием из $\mathrm{GL}(2 n, \mathbb{Z})$, сохраняющим форму $(6)$.

Уточним сказанное. Группа $\operatorname{SL}(\delta, \mathbb{Z})$ является подгруппой $\operatorname{GL}(2 n, \mathbb{Z})$ и состоит из элементов $g$ таких, что

$$
\left(\begin{array}{cc}
A & B \\
C & D
\end{array}\right) \cdot\left(\begin{array}{cc}
0 & \Delta_{\delta} \\
-\Delta_{\delta} & 0
\end{array}\right) \cdot\left(\begin{array}{ll}
A^{*} & C^{*} \\
B^{*} & D^{*}
\end{array}\right)=\left(\begin{array}{cc}
0 & \Delta_{\delta} \\
-\Delta_{\delta} & 0
\end{array}\right),
$$

где

$$
g=\left(\begin{array}{ll}
A & B \\
C & D
\end{array}\right)
$$

и $A, B, C$ и $D$ - целочисленные $(n \times n)$-матрицы.

Действие $\operatorname{Sp}(\delta, \mathbb{Z})$ на $\mathscr{H}_{n}$, порожденное автоморфизмами решеток, имеет вид

$$
\Omega \rightarrow\left(A \Omega+B \Delta_{\delta}\right) \cdot\left(\Delta_{\delta}^{-1} C \Omega+\Delta_{\delta}^{-1} D \Delta_{\delta}\right)^{-1} .
$$

При $n=1, \delta=1$ мы получаем обычное модулярное действие группы $\operatorname{SL}(2, \mathbb{Z})$ на верхней полуплоскости.

1.4.1. Пространством модулей $n$-мерных абелевых многообразий с поляризаиией типа $\delta$ является фактор-пространство верхней полуплоскости Зигеля $\mathscr{H}_{n}$ по действию (16) группьь $\mathrm{Sp}(\delta, \mathbb{Z})$ :

$$
\mathscr{A}_{n}^{\delta}=\mathscr{H}_{n} / \operatorname{Sp}(\delta, \mathbb{Z}) .
$$

Изогенией абелевых многообразий $M$ и $M^{\prime}$ назьвается голоморфное конечнолистное накрытие $\varphi: M \rightarrow M^{\prime}$.

1.4.2. Для любого поляризованного абелева многообразия $M$ существует изогенное ему главно поляризованное абелево многообразие $M^{\prime}$ :

$$
\varphi: M \rightarrow M^{\prime}
$$

причем степень накрытия равна $\Delta=\delta_{1} \cdots \delta_{n}$, где $\left(\delta_{1}, \ldots, \delta_{n}\right)-$ тип поляризачии.

Докажем предложение 1.4.2. Пусть $M$ - абелево многообразие $M=\mathbb{C}^{n} / \Lambda$ вида (7), $\left(\lambda_{1}, \ldots, \lambda_{2 n}\right)$ - базис решетки $\Lambda$, а ходжева форма $\omega$ имеет в координатах, отвечающих этому базису, вид (7).

Рассмотрим решетку $\Lambda^{\prime}$, порожденную векторами базиса (8). Она содержит $\Lambda$ как подрешетку индекса $\Delta$. Проекщия

$$
\varphi: \mathbb{C}^{n} / \Lambda \rightarrow \mathbb{C}^{n} / \Lambda^{\prime}
$$

является изогенией степени $\Delta$. Выберем форму $\omega^{\prime}$ такую, что $\varphi^{*}\left(\omega^{\prime}\right)=\omega$. Она задает согласно (10) главную поляризацию на $M^{\prime}$. Предложение 1.4 .2 доказано. 


\section{§ 2. Линейные расслоения на комплексных торах}

2.1. Линейные расслоения, группа Пикара, классы Чжэня, положительные расслоения.

Линейным расслоением $L$ над комплексным многообразием $M$ назьвается векторное расслоение со слоем $\mathbb{C}$ и голоморфными функциями перехода, а именно:

1) определена проекция $p: L \rightarrow M$;

2) существует открытое покрытие $\mathscr{U}=\left\{U_{\alpha}\right\}$ пространства $M$ такое, что над каждьм элементом этого покрытия расслоение тривиально:

$$
U_{\alpha} \times \mathbb{C} \stackrel{\xi_{\alpha}}{\approx} p^{-1}\left(U_{\alpha}\right), \quad p \cdot \xi_{\alpha}(z, v)=z \in U_{\alpha} \subset M
$$

3) функции перехода

$$
g_{\alpha \beta}(z)=\left.\xi_{\alpha}^{-1} \xi_{\beta}\right|_{p^{-1}(z)}: U_{\alpha} \cap U_{\beta} \rightarrow \mathbb{C}^{*}=\mathbb{C} \backslash\{0\}
$$

голоморфны.

Функции перехода по определению удовлетворяют условиям

$$
g_{\alpha \beta} \cdot g_{\beta \alpha}=1, \quad g_{\alpha \beta} \cdot g_{\beta \gamma} \cdot g_{\gamma \alpha}=1
$$

из которых следует, что набор функций $g_{\alpha \beta}$, однозначно задающий линейное расслоение, является 1-коциклом из $C^{1}\left(\mathscr{U}, \mathscr{O}^{*}\right)$. Пучок $\mathscr{O}^{*}$ определяется своими ограничениями следуюшим образом: $\mathscr{O}^{*}(U)$ - группа (относительно умножения) всюду отличных от нуля голоморфных функций на $U \subset M$. Два коцикла $g$ и $g^{\prime}$ когомологич$\boldsymbol{H b l}$ (по определению), если существует такой набор сечений $f_{\alpha}: U_{\alpha} \rightarrow \mathscr{O}^{*}\left(U_{\alpha}\right)$, что $g_{\alpha \beta}^{\prime}=\left(f_{\alpha} / f_{\beta}\right) g_{\alpha \beta}$.

Линейные расслоения назьваются эквивалентным.ми, если отвечающие им кощиклы $g$ и $g^{\prime}$ когомологичны (функции $f_{\alpha}$ деформируют тривиализации: $\xi_{\alpha} \rightarrow \xi_{\alpha} / f_{\alpha}$ ).

Классы эквивалентности линейных расслоений образуют коммутативную группу - группу Пикара $\operatorname{Pic}(M)$ - относительно тензорного умножения

$$
L, L^{\prime} \rightarrow L \otimes L^{\prime}, \quad g_{\alpha \beta}, g_{\alpha \beta}^{\prime} \rightarrow g_{\alpha \beta} \cdot g_{\alpha \beta}^{\prime}
$$

и перехода к двойственному расслоению

$$
L \rightarrow L^{*}, \quad g_{\alpha \beta} \rightarrow g_{\alpha \beta}^{-1}
$$

Из сказанного вьше следует:

2.1.1. СТРУКтУРА ГРУППЫ ПИКАРА. Группа Пикара линейных расслоений на $M$ изоморфна одномерной группе когомологий $M c$ коэффициентами в пучке ростков ненулевых голоморфных функиий: $\operatorname{Pic}(M)=H^{1}\left(M ; \mathscr{O}^{*}\right)$.

Линейному расслоению $L$ отвечает его класс Чжэня: $c_{1}(L)$. Мы дадим его определение через функции перехода $g_{\alpha \beta}$. Пусть $\mathscr{U}$ - открытое покрытие $M$, состояшее из 
односвязных областей в $M$, над которьми расслоение $L$ тривиально, и $\left\{g_{\alpha} \beta\right\}$ - набор функций перехода. Определим функции

$$
h_{\alpha \beta}=\frac{1}{2 \pi \sqrt{-1}} \log \left(g_{\alpha \beta}\right), \quad \log \left(g_{\alpha \beta}\right)=-\log \left(g_{\beta \alpha}\right) .
$$

По ним построим коцикл $z_{\alpha \beta \gamma} \in C^{2}(\mathscr{U} ; \mathbb{Z})$ :

$$
z_{\alpha \beta \gamma}=h_{\alpha \beta}+h_{\beta \gamma}-h_{\gamma \alpha}
$$

Класс когомологий $c_{1}(L) \in H^{2}(M ; \mathbb{Z})$, реализуемый этим коциклом, назьвается ( $n е p$ вылм) классом Чжсэня линейного расслоения $L$.

Это определение корректно, так как выбор других ветвей логарифма при определении $h_{\alpha \beta}\left(\log \left(g_{\alpha \beta}\right) \rightarrow \log \left(g_{\alpha \beta}\right)+2 \pi \sqrt{-1} k_{\alpha \beta}, k_{\alpha \beta} \in \mathbb{Z}\right)$ эквивалентен замене коцикла $z_{\alpha \beta \gamma}$ на когомологичный ему: $z \rightarrow z+d k$.

Из (20) следует, что класс Чжэня $c_{1}$ является гомоморфизмом групшы Пикара в $H^{2}(M ; \mathbb{Z})$ :

$$
c_{1}\left(L \otimes L^{\prime}\right)=c_{1}(L)+c_{1}\left(L^{\prime}\right), \quad c_{1}\left(L^{*}\right)=-c_{1}(L)
$$

Переизложим определение $c_{1}$ в инвариантной форме. Рассмотрим точную последовательность пучков

$$
0 \rightarrow \mathbb{Z} \rightarrow \mathscr{O} \stackrel{\exp }{\rightarrow} \mathscr{O}^{*} \rightarrow 0
$$

где $\mathbb{Z}$ - постоянный пучок, $\mathscr{O}$ - пучок ростков голоморфных функщий, а ехр - экспоненциальньй гомоморфизм $f \rightarrow \exp (2 \pi \sqrt{-1} f)$. Ему отвечает точная когомологическая последовательность

$$
\cdots \rightarrow H^{k}(M ; \mathbb{Z}) \rightarrow H^{k}(M ; \mathscr{O}) \rightarrow H^{k}\left(M ; \mathscr{O}^{*}\right) \rightarrow H^{k+1}(M ; \mathbb{Z}) \rightarrow \cdots
$$

Рассмотрим граничньй гомоморфизм этой последовательности при $k=1$ :

$$
\delta: H^{1}\left(M ; \mathscr{O}^{*}\right)=\operatorname{Pic}(M) \rightarrow H^{2}(M ; \mathbb{Z}) .
$$

Его образ является по определению первьм классом Чжэня:

$$
\delta(L)=c_{1}(L) .
$$

Линейное расслоение $L$ назьвают положительнылм, если его первьй класс Чжэня реализуется ходжевой формой $\omega: c_{1}(L)=[\omega]$.

\section{2. Классы Чжэня линейных расслоений на торах.}

Пусть $M=\mathbb{C}^{n} / \Lambda$ - комплексньй тор и $\pi: \mathbb{C}^{n} \rightarrow M-$ универсальное накрытие. Любое линейное расслоение $L$ на $M$ имеет свой обратный образ $\pi^{*}(L)$ - линейное расслоение на $\mathbb{C}^{n}$. Согласно $\bar{\partial}$-лемме Пуанкаре $H^{1}\left(\mathbb{C}^{n} ; \mathscr{O}^{*}\right)=0$ (см., например, [41]). Поэтому расслоение $\pi^{*}(L)$ тривиально: $\pi^{*}(L)=\mathbb{C}^{n} \times \mathbb{C}$.

Зафиксируем какую-то тривиализацию расслоения $\pi^{*}(L)$. Сравнивая тривиализации расслоения $\pi^{*}(L)$ в точках $z$ и $z+\lambda(\lambda \in \Lambda)$, мы построим автоморфизмы слоя

$$
e_{\lambda}(z): \mathbb{C} \rightarrow \mathbb{C}, \quad e_{\lambda}(z) \in \mathbb{C}^{*}
$$


Совокупность этих автоморфизмов образует множество мультипликаторов расслоения $L$ : набор функций $\left\{e_{\lambda} \in \mathscr{O}^{*}(U)\right\}_{\lambda \in \Lambda}$ такой, что

$$
e_{\lambda^{\prime}}(z+\lambda) e_{\lambda}(z)=e_{\lambda}\left(z+\lambda^{\prime}\right) e_{\lambda^{\prime}}(z)=e_{\lambda+\lambda^{\prime}}(z) .
$$

Любой набор функций $\left\{e_{\lambda} \in \mathscr{O}^{*}(U)\right\}_{\lambda \in \Lambda}$, удовлетворяющий (23), задает линейное расслоение, которое получается из $\mathbb{C}^{n} \times \mathbb{C}$ факторизацией по действию групшы $\Lambda$ :

$$
(z, v) \rightarrow\left(z+\lambda, e_{\lambda}(z) \cdot v\right), \quad z \in \mathbb{C}^{n}, \quad v \in \mathbb{C} .
$$

Два набора мультипликаторов $e_{\lambda}$ и $\widetilde{e}_{\lambda}$ задают эквивалентные расслоения тогда и только тогда, когда существует голоморфная функция $\varphi: \mathbb{C} \rightarrow \mathbb{C}^{*}$ такая, что

$$
\widetilde{e}_{\lambda}(z)=e_{\lambda}(z) \varphi(z+\lambda) \varphi^{-1}(z) .
$$

Функция $\varphi$ изменяет тривиализацию $\pi^{*}(L)$.

Найдем формулу, выражающую класс Чжэня через мультипликаторы расслоения. Выберем базис $\lambda_{1}, \ldots, \lambda_{2 n}$ решетки $\Lambda$ и обозначим через $x^{1}, \ldots, x^{2 n}$ отвечаюшие ему вешественные координаты на $\mathbb{C}^{n}$. Рассмотрим покрытие тора $M$ множествами

$$
U_{\lambda}=\left\{\left|x^{k}-\lambda^{k}\right| \leqslant \frac{3}{4}\right\},
$$

где $\lambda=\left(\lambda^{1}, \ldots, \lambda^{2 n}\right) \in \Lambda$. Функции перехода согласно определению мультипликаторов примут вид

$$
g_{\alpha \beta}(z)=e_{\alpha-\beta}(z+\beta) .
$$

Определим функции $f_{\alpha}(z)$ по формуле

$$
e_{\alpha}(z)=e^{2 \pi \sqrt{-1}} f_{\alpha}(z) .
$$

Рассмотрим теперь, как по коциклу (20) построить реализующую его 2-форму. Нерв $N(\mathscr{U})$ минимального подпокрытия построенного вьше покрытия $U_{\alpha}$ гомеоморфен тору $M$, и его когомологии с коэффициентами в $\mathbb{Z}$ совпадают с $H^{*}(M ; \mathbb{Z})$. Формула (20) задает значение коцикла $z$ на двумерном симплексе $(\alpha, \beta, \gamma) \in N(\mathscr{U})$.

Пусть $\tau, \sigma \in \Lambda$. Натянем на них параллелограмм $\Pi_{\tau, \sigma}$, которьй проектируется в тор $T_{\tau, \sigma} \subset M$. Зная значения $z$ на двумерных симплексах, определим значение $c_{1}$ на этом двумерном торе.

Согласно (20) и (24)-(25)

$$
c_{1}\left(\left[T_{\tau, \sigma}\right]\right)=f_{\sigma}(z)+f_{\tau}(z+\sigma)-f_{\tau}(z)-f_{\sigma}(z+\tau) .
$$


2.2.1. Формула (27) определяет класс Чжсәня $c_{1}$ линейного расслоения на торе $M=\mathbb{C}^{n} / \Lambda$. Класс Чжәэя задает кососимметрическую целочисленную

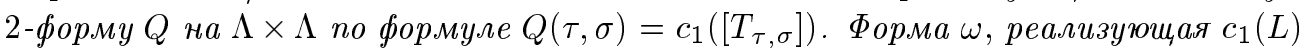
(m.e. $\left.[\omega]=c_{1}(L)\right)$, имеет вид

$$
\omega=\frac{1}{2} Q\left(\lambda_{k}, \lambda_{l}\right) d x^{k} \wedge d x^{l}
$$

Следующее утверждение дает интерпретацию класс Чжэня как реализуемого формой кривизны линейного расслоения (см. [17]).

$(p, q)$-формой называется линейная комбинация дифференщиальных форм вида $f d z^{i_{1}} \wedge \cdots \wedge d z^{i_{p}} \wedge d \bar{z}^{j_{1}} \wedge \cdots \wedge d \bar{z}^{j_{q}}$. Для компактного комплексного многообразия $M$ выделим в $H^{2}(M ; \mathbb{Z})$ подгруппу $H_{1,1}^{2}(M ; \mathbb{Z})$, образованную элементами, которые реализуются $(1,1)$-формами.

2.2.2. Первый класс Чжээя линейного расслоения $L$ на компактном комплексном пространстве $M$ лежит в $H_{1,1}^{2}(M ; \mathbb{Z}): c_{1}(L) \in H_{1,1}^{2}(M ; \mathbb{Z})$.

Мы дадим доказательство предложения 2.2.2 по модулю теоремы де Рама о сравнении пучковых когомологий и когомологий де Рама. Для торов эквивалентность (27) и (29) проверяется прямьми вычислениями.

Выберем на линейном расслоении эрмитову метрику, т.е. зададим положительные функции (метрический тензор) $a_{\alpha}: U_{\alpha} \rightarrow \mathbb{R}$ такие, что

$$
a_{\alpha} \cdot\left|g_{\alpha \beta}\right|^{2}=a_{\beta} \quad \text { на } \quad U_{\alpha} \cap U_{\beta}
$$

Пусть $d=d^{\prime}+d^{\prime \prime}$ - разложение оператора внешнего дифференцирования $d$ на голоморфную и антиголоморфную части, где $d^{\prime}$ и $d^{\prime \prime}$ переводят $(p, q)$-форму в $(p+1, q)$-форму и $(p, q+1)$-форму, соответственно. Очевидно, что $d^{\prime 2}=d^{\prime \prime 2}=0$. Применим оператор $d^{\prime}$ к логарифмам обеих частей (28) и получим с учетом голоморфности функций перехода:

$$
d^{\prime} \log \left(a_{\alpha}\right)+d^{\prime} \log \left(g_{\alpha \beta}\right)=d^{\prime} \log \left(a_{\beta}\right) .
$$

Tак как $d d^{\prime} \log \left(g_{\alpha \beta}\right)=0$, то форма $d d^{\prime} \log \left(a_{\alpha}\right)$ корректно определена на всем многообразии $M$.

Из сравнения когомологий де Рама со спектральными когомологиями и (20) следует, что

$$
c_{1}(L)=\left[\frac{1}{2 \pi \sqrt{-1}} d^{\prime \prime} d^{\prime} \log \left(a_{\alpha}\right)\right]
$$

Предложение 2.2.2 доказано.

2.3. Построение линейных расслоений на торах с заданными классами Чжэня. 
2.3.1. СтруктУРА ГРУППЫ $H_{1,1}^{2}(M ; \mathbb{Z})$. Если $M=\mathbb{C}^{n} / \Lambda$ - комплексный тор, то әруппа $H_{1,1}^{2}(M ; \mathbb{Z})$ изоморфна группе кососимметрических билинейных (над $\left.\mathbb{R}\right)$ 2-форм $Q$ на $\mathbb{C}^{n} \times \mathbb{C}^{n}$ таких, что $Q$ челочисленна на $\Lambda \times \Lambda$ и

$$
Q(u, v)=Q(\sqrt{-1} u, \sqrt{-1} v) .
$$

Согласно предложениям 1.3.2 и 1.3.3 можно ограничиться 2 -формами $\omega \in H^{2}(M ; \mathbb{Z})$ с постоянными коэффициентами и каждой 2-форме $\omega$ сопоставить целочисленную кососимметрическую билинейную форму $Q$ на $\Lambda \times \Lambda$. После $\mathbb{R}$-линейного распространения на $\mathbb{C}^{n} \times \mathbb{C}^{n}$ форма $Q$ совпадет с $\omega$.

Пусть $Q=\alpha_{i j} d z^{i} \wedge d z^{j}+\beta_{i j} d z^{i} \wedge d \bar{z}^{j}+\gamma_{i j} d \bar{z}^{i} \wedge d \bar{z}^{j}$. Очевидно, что $Q$ удовлетворяет (30) тогда и только тогда, когда $\alpha_{i j}=\gamma_{i j}=0$. Последнее эквивалентно тому, что $\omega \in H_{1,1}^{2}(M ; \mathbb{Z})$. Предложение 2.3.1 доказано.

Пусть $\omega$ - 2-форма на торе $M=\mathbb{C}^{n} / \Lambda$, реализующая элемент из $H_{1,1}^{2}(M ; \mathbb{Z})$, и $Q$ ассоциирована с $\omega$ (см. предложение 1.3.3).

Из (30) следует, что $\mathbb{R}$-билинейная форма

$$
H(u, v)=-\pi(Q(\sqrt{-1} u, v)+\sqrt{-1} Q(u, v))
$$

является эрмитовой, т.е.

$$
H(u, v)=\overline{H(v, u)}, \quad H(\alpha u, v)=\alpha H(u, v), \quad \alpha \in \mathbb{C} .
$$

В случае, когда форма $\omega$ является ходжевой формой с постоянными коэффициентами, метрика $H$ является ассоциированной с ней кэлеровой метрикой.

Определим теперь функции $e_{\lambda}(z), \lambda \in \Lambda, z \in \mathbb{C}^{n}$, следуюшим образом:

$$
e_{\lambda}(z)=\alpha(\lambda) \exp \left(H(z, \lambda)+\frac{1}{2} H(\lambda, \lambda)\right) \text {. }
$$

2.3.2. Функции (31) тогда и только тогда задают семейство мультипликаторов линейного расслоения $L$ на $M=\mathbb{C}^{n} / \Lambda$, когда выполняется соотношение

$$
\frac{\alpha\left(\lambda+\lambda^{\prime}\right)}{\alpha(\lambda) \alpha\left(\lambda^{\prime}\right)}=\exp \left(\pi \sqrt{-1} Q\left(\lambda, \lambda^{\prime}\right)\right) \quad \text { для } \quad \text { всех } \quad \lambda, \lambda^{\prime} \in \Lambda .
$$

Если (32) выполнено, то первый класс Чжсэня расслоения L совпадает с $[\omega]$.

Предложение 2.3.2 выводится прямьми вычислениями из (23) и (26).

Из 2.3.2 вытекает следующее предложение.

2.3.3. Для любого әлемента $[\omega] \in H_{1,1}^{2}(M ; \mathbb{Z})$ существует линейное расслоение $L$ на $M=\mathbb{C}^{n} / \Lambda$, заданное мультипликаторами вида (31) и такое, что $c_{1}(L)=[\omega]$.

Для доказательства достаточно показать, что для любой кососимметрической формы $Q$, которая целочисленна на $\Lambda \times \Lambda$, соотношения (32) разрешимы.

Как и в 1.3 .5 , построим такой базис $\lambda_{1}, \ldots, \lambda_{2 n}$ решетки $\Lambda$, что форма $Q$ примет в нем вид (6). На каждом подпространстве $V_{k}=\mathbb{R}\left\{\lambda_{k}, \lambda_{n+k}\right\}$ определим функцию $\alpha_{k}$ следуюшим образом: $\alpha_{k}\left(x \lambda_{k}+y \lambda_{n+k}\right)=\exp \left(\pi \sqrt{-1} \delta_{k} x y\right)$. Определим теперь функцию $\alpha(v)=\alpha_{1}\left(v_{1}\right) \cdots \alpha_{n}\left(v_{n}\right)$, где $v=v_{1}+\cdots+v_{n}, v_{k} \in V_{k}$. Осталось заметить, что функция $\alpha$ удовлетворяет (32). Предложение 2.3.3 доказано.

2.4. Линейные расслоения с $c_{1}=0$ : группа $\operatorname{Pic}^{0}(M)$. 
2.4.1. Если линейное расслоение на комплексном торе $M$ имеет нулевой класс Чжсэня, то оно может быть задано постоянными мультипликаторамu.

Докажем это предложение. Пусть $c_{1}(L)=0$. Из определения класса Чжэня $c_{1}$ и точной последовательности $(22)$ следует, что $[L] \in H^{1}\left(M ; \mathscr{O}^{*}\right)$ лежит в образе гомоморфизма $H^{1}(M ; \mathscr{O}) \rightarrow H^{1}\left(M ; \mathscr{O}^{*}\right)$. Действительно, коцикл $\zeta \in H^{1}(M ; \mathscr{O})$ задается сечениями $\log \left(g_{\alpha \beta}\right)$ и переходит при этом в коцикл $g_{\alpha \beta}$, задаюший расслоение $L$.

Для любого компактного кэлерова многообразия

1) группа $H^{1}(M ; \mathscr{O})$ изоморфина подгруппе $H^{1}(M ; \mathbb{C})$, состояшей из классов когомологий замкнутых $(0,1)$-форм;

2) естественный гомоморфизм $H^{1}(M ; \mathbb{C}) \rightarrow H^{1}(M ; \mathscr{O})$, порожденный вложением пучков $\mathbb{C} \rightarrow \mathscr{O}$, эпиморфен. На языке когомологий де Рама он состоит в проектировании

$$
\xi=\xi^{1,0}+\xi^{0,1} \rightarrow \xi^{0,1},
$$

где $\xi=\xi^{1,0}+\xi^{0,1}-$ разложение замкнутой формы в сумму $(1,0)-$ и $(0,1)$-форм (см. [17]).

Отсюда следует, что коцикл $\zeta$ когомологичен коциклу с постоянными коэффициентами $\log \left(g_{\alpha \beta}\right)$. Это эквивалентно тому, что линейное расслоение задается постоянными мультипликаторами. Предложение 2.4.1 доказано.

Рассмотрим линейные расслоения с постоянными мультипликаторами. Согласно предложениям 2.3.2 и 2.4.1 с точностью до эквивалентности это расслоения с $c_{1}=0$.

2.4.2. Классы әквивалентности расслоений на торе $M$ с $c_{1}=0$ параметризуются точками двойственного тора $\widehat{M}=\operatorname{Pic}^{0}(M)$.

Пусть $L$ - расслоение с постоянными мультипликаторами $e_{\lambda}$. Легко построить такую функцию $\varphi(z)$ вида $\varphi(z)=\exp \left(a_{k} z^{k}\right)$, что $|\varphi(\lambda)|=\left|e_{\lambda}\right|^{-1}$. Согласно (24) мультипликаторы $\widetilde{e}_{\lambda}(z)=e_{\lambda} \varphi(z+\lambda) \varphi(z)^{-1}$ задают то же расслоение $L$, постоянны и $\left|\widetilde{e}_{\lambda}\right|=1$. Такие наборы мультипликаторов находятся во взаимно однозначном соответствии с гомоморфизмами

$$
\psi: \Lambda \rightarrow \mathbb{C}_{1}=\{z \in \mathbb{C}:|z|=1\}
$$

Это соответствие задается формулой

$$
\psi(\lambda)=e_{\lambda}
$$

Гомоморфизмы (33) параметризуются точками тора $\widehat{M}=\operatorname{Hom}\left(\Lambda, \mathbb{C}_{1}\right)$.

Различные гомоморфизмы определяют неэквивалентные расслоения. Докажем это от противного. Пусть $\psi_{1}$ и $\psi_{2}$ задают эквивалентные расслоения. Тогда согласно $(24)$ сушествует целая функция $\varphi(z)$ такая, что $\psi_{1}(\lambda)=\psi_{2}(\lambda) \varphi(z+\lambda) \varphi^{-1}(z)$ для любых $z \in \mathbb{C}^{n}$ и $\lambda \in \Lambda$. Так как $\left|\psi_{1}(\lambda)\right|=\left|\psi_{2}(\lambda)\right|=1$, то функция $\varphi(z)$ ограниченна и согласно теореме Лиувилля постоянна. Значит, $\psi_{1}=\psi_{2}$. Предложение 2.4 .2 доказано.

Из предложения 2.4.2 и соответствия (34) следует, что подгруппа $\mathrm{Pic}^{0}(M)$, состо- $^{\circ}$ ящая из классов эквивалентности расслоений с $c_{1}=0$, совпадает с $\widehat{M}=\operatorname{Hom}\left(\Lambda, \mathbb{C}_{1}\right)$ 
и как группа. Так как $H^{0}\left(M ; \mathscr{O}^{*}\right)=0$, то из точной последовательности $(24)$ следует другое определение тора $\operatorname{Pic}^{0}(M)$.

2.4.3. $\operatorname{Pic}^{0}(M)=H^{1}(M ; \mathscr{O}) / H^{1}(M ; \mathbb{Z})$.

Из предложений 2.3.2, 2.3.3 и 2.4.2 вытекает следуюшая теорема.

2.4.4. Теорема АПпеля-Эмьера. 1) Любое линейное расслоение на комплексном торе $M$ может быть задано мультипликаторами вида (31);

2) Последовательность

$$
0 \rightarrow \widehat{M}=\operatorname{Pic}^{0}(M) \rightarrow \operatorname{Pic}(M) \rightarrow H_{1,1}^{2}(M ; \mathbb{Z}) \rightarrow 0,
$$

где первый гомоморфизм является вложением, а второй сопоставляет расслоению его первый класс Чжэня, точна.

\section{5. Положительные линейные расслоения на торах и их сечения.}

Пусть $M=\mathbb{C}^{n} / \Lambda$ - тор, на котором задана ходжева форма $\omega$. Согласно предложениям 1.3 .5 и 1.3 .6 существует такой базис $\mathscr{V}=\left(\lambda_{1}, \ldots, \lambda_{2 n}\right)$ решетки $\Lambda$, что

$1)$ в координатах, отвечаюших базису $\mathscr{V}$, форма $\omega$ имеет вид $(7)$, где $\delta_{j} \in \mathbb{Z}$, $\delta_{j}>0$

$2)$ векторы $\lambda_{1} / \delta_{1}, \ldots, \lambda_{n} / \delta_{n}$ образуют комплексный базис в $\mathbb{C}^{n}$ (обозначим отвечающие им координаты через $z^{1}, \ldots, z^{n}$ );

$3)$ в координатах $\left(z^{1}, \ldots, z^{n}\right)$ решетка $\Lambda$ имеет вид $\Delta_{\delta} N_{1}+\Omega N_{2}, N_{i} \in \mathbb{Z}^{n}$, где матрища $\Omega$ симметрична и ее мнимая часть положительно определена.

Пространство модулей $\mathscr{L}(M,[\omega])$ линейных расслоений на $M$ с $c_{1}=[\omega]$ согласно теореме Аппеля-Эмбера изоморфно тору $\operatorname{Pic}^{0}(M)$ : если $L, L^{\prime} \in \mathscr{L}(M,[\omega])$, то сушествует и единственно расслоение $L^{\prime \prime} \in \operatorname{Pic}^{0}(M)$ такое, что $L^{\prime}=L \otimes L^{\prime \prime}$.

В то же время тор $M$ действует на $\mathscr{L}(M,[\omega])$ сдвигами: пусть $L \in \mathscr{L}(M,[\omega])$ и $\tau_{\mu}: M \rightarrow M-$ сдвиг $z \rightarrow z+\mu$, тогда определено расслоение $\tau_{\mu}^{*}(L)$. На языке мультипликаторов это действие описьвается просто: $e_{\lambda}(z) \rightarrow e_{\lambda}^{\prime}(z)=e_{\lambda}(z+\mu)$.

Пусть расслоение $L \in \mathscr{L}(M,[\omega])$ задано мультипликаторами

$$
e_{\lambda_{k}}=1, \quad e_{\lambda_{n+k}}=\exp \left(-2 \pi \sqrt{-1} z^{k}\right), \quad k=1, \ldots, n .
$$

Оно лежит в $\mathscr{L}(M,[\omega])$. Тогда расслоение $\tau_{\mu}^{*}(L) \otimes L^{*} \in \mathscr{L}(M, 0)$ задается постоянными мультипликаторами

$$
\widetilde{e}_{\lambda_{k}}^{(\mu)}=1, \quad \widetilde{e}_{\lambda_{n+k}}^{(\mu)}=\exp \left(-2 \pi \sqrt{-1} \mu^{k}\right), \quad k=1, \ldots, n,
$$

и из (37) прямыми вычислениями выводится

2.5.1. Пусть $L \in \mathscr{L}(M,[\omega])$. Тогда отображение

$$
\tau: M \rightarrow \operatorname{Pic}^{0}(M), \quad \mu \stackrel{\tau}{\rightarrow} \tau_{\mu}^{*}(L) \otimes L^{*},
$$


является гомоморфизмом на всю группу $\operatorname{Pic}^{0}(M)$, ядро которого изоморфно подрешетке, порожденной базисом $\left\{\delta_{k}^{-1} \lambda_{k}, \delta_{k}^{-1} \lambda_{n+k}\right\}$. Любое расслоение $L \in \mathscr{L}(M, 0)$ представимо в виде $\tau_{\mu}^{*}(L) \otimes L^{*}$.

Обозначим через $\mathscr{O}(L)$ пучок ростков голоморфных сечений расслоения $L$. Глобальные сечения находятся во взаимно однозначном соответствии с целыми функциями $f(z)$, удовлетворяюшими условиям периодичности

$$
f(z+\lambda)=e_{\lambda}(z) f(z),
$$

где $e_{\lambda}$ - мультипликаторы, задаюшие расслоение $L$. Глобальные сечения образуют векторное пространство $H^{0}(M ; \mathscr{O}(L))$.

2.5.2. Если расслоение $L$ положительно и $c_{1}=[\omega]$, то

$$
h^{0}(L)=\operatorname{dim}_{\mathbb{C}} H^{0}(M ; \mathscr{O}(L))=\delta_{1} \cdot \ldots \cdot \delta_{n},
$$

где $\left(\delta_{1}, \ldots, \delta_{n}\right)-$ тип поляризачии формы $\omega$.

ДОКАЗАТЕЛЬСТВО ПРЕДЛОЖЕНИЯ 2.5.2. Так как сдвиГ на $\mu$ гомотопен тождественному отображению и любое расслоение из $\mathscr{L}(M,[\omega])$ получается сдвигом, то достаточно найти $h^{0}(L)$ для произвольного расслоения $L \in \mathscr{L}(M,[\omega])$. Рассмотрим расслоение с мультипликаторами (36). Так как $e_{\lambda_{k}} \equiv 1$, то каждое сечение $f$ разлагается в ряд Фурье по $\exp \left(2 \pi \sqrt{-1} l_{k} \delta_{k}^{-1} z^{k}\right)$ :

$$
f(z)=\sum_{l \in \mathbb{Z}^{n}} a_{l} \exp \left(2 \pi \sqrt{-1} \sum_{k} l^{k} \delta_{k}^{-1} z^{k}\right) .
$$

Сравнив разложения Фурье функции $f\left(z+\lambda_{n+k}\right)$ с разложением Фурье функции $f(z)$, мы с учетом (39) получаем

$$
a_{l+d_{k}}=\exp \left(2 \pi \sqrt{-1}\left\langle l, \Delta_{\delta}^{-1} \lambda_{n+k}\right\rangle\right) a_{l},
$$

где $\langle u, v\rangle=\sum_{k} u^{k} v^{k}$, а $d_{k} \in \mathbb{Z}^{n}, d_{k}^{k}=\delta_{k}, d_{k}^{j}=0$ при $j \neq k$.

Из (41) следует, что функция $f$ полностью определяется значениями $a_{l}$ при $l \in$ $\Pi_{\delta}=\left\{0 \leqslant l^{k}<\delta_{k}\right\}$. Отсюда вытекает, что $h^{0}(L) \leqslant \delta_{1} \cdot \ldots \cdot \delta_{n}$.

Рассмотрим функции $\theta_{\bar{l}}, \bar{l} \in \Pi_{\delta}$, вида (40)-(41) и такие, что

$$
a_{l}=\left\{\begin{array}{cc}
0 & \text { при } l \in \Pi_{\delta}, \\
1 & \text { при } l=\bar{l} .
\end{array}\right.
$$

Согласно (40)-(41) для каждого $\bar{l} \in \Pi_{\delta}$ ряд (40) определен однозначно. Все эти ряды определяют целые функции. Мы объясним это в $§ 3$ на примере тэта-функции главно поляризованного ходжева тора.

Очевидно, что функции $\theta_{\bar{l}}$ линейно независимы как сечения $\mathscr{O}(L)$. Значит, $h^{0}(L)=$ $\delta_{1} \cdot \ldots \cdot \delta_{n}$. Предложение 2.5 .2 доказано.

Из предложений 2.5.1 и 2.5.2 вытекает следующее утверждение.

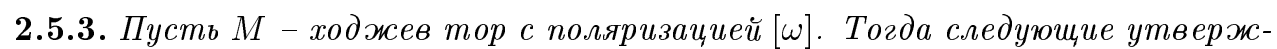
дения әквивалентны:

1) поляризация $[\omega]-$ главная;

2) гомоморфизм (38) $\tau: M \rightarrow \widehat{M}$ является изоморфизмом;

3) $h^{0}(L)=1$, где $c_{1}(L)=[\omega]$. 


\section{§3. Тэта-функции}

3.1. Тэта-функция главно поляризованного ходжева тора.

Любой ходжев тор $M$ с главной поляризацией $[\omega]$ представим в следующем виде:

1) $M=\mathbb{C}^{n} / \Lambda$, где в координатах по отношению к базису $\left(\lambda_{1}, \ldots, \lambda_{2 n}\right)$ решетки $\Lambda$ форма $\omega$ имеет вид $\omega=\sum_{k} d x^{k} \wedge d x^{n+k}$;

$2)$ решетка $\Lambda$ в комплексных координатах, связанных с базисом $\left(\lambda_{1}, \ldots, \lambda_{n}\right)$ в $\mathbb{C}^{n}$, имеет вид $\left\{\mathbb{Z}^{n}+\Omega \mathbb{Z}^{n}\right\}$

3) $(n \times n)$-матрища $\Omega$ симметрична и ее мнимая часть $\operatorname{Im} \Omega$ положительно определена.

Это следует из предложений 1.3.5 и 1.3.6 и критерия Римана. В дальнейшем мы под главно поляризованньм ходжевым тором будем всегда подразумевать комплексньй тор, уже представленный в таком виде.

Пусть $L$ - расслоение, заданное мультипликаторами

$$
e_{\lambda_{k}} \equiv 1, \quad e_{\lambda_{n+k}}=\exp \left(-\pi \sqrt{-1} \Omega_{k k}-2 \pi \sqrt{-1} z^{k}\right)
$$

Здесь $\Omega_{i j}=\Omega_{j}^{i}, \lambda_{n+k}=\Omega e_{k}, k=1, \ldots, n$.

Согласно $(27) c_{1}(L)=[\omega]$ и любое расслоение с таким же классом Чжэня может быть получено из $L$ сдвигом (предложение 2.5.1). Поэтому мы ограничимся построением глобальных сечений данного расслоения. Это пространство одномерно согласно предложению 2.5.2.

Рассмотрим формальный ряд

$$
\theta(z, \Omega)=\sum_{m \in \mathbb{Z}^{n}} \exp (\pi \sqrt{-1}\langle\Omega m, m\rangle+2 \pi \sqrt{-1}\langle m, z\rangle)
$$

Так как мнимая часть матрицы $\Omega$ положительно определена, то для каждого компактного подмножества $U \subset \mathbb{C}^{n}$ сушествует постоянная $c(U)$ такая, что

$$
|\exp (\pi \sqrt{-1}\langle\Omega m, m\rangle+2 \pi \sqrt{-1}\langle m, z\rangle)|<c(U) \exp \left(-d|m|^{2}\right)
$$

где $d$ - положительная постоянная, зависящая от $\Omega$. Отсюда следует, что ряд (44) абсолютно сходится на любом компакте $U \subset \mathbb{C}^{n}$ и определяет целую функцию.

ОПРЕДЕЛЕНИЕ. Тәта-функиией $\theta(z, \Omega)$ называется функция, определяемая рядом (44).

В дальнейшем под $\theta(z)$ понимается функция $\theta(z, \Omega)$.

Из (44) следует, что тэта-функция удовлетворяет условиям периодичности

$$
\begin{gathered}
\theta(z+m, \Omega)=\theta(z, \Omega), \\
\theta(z+\Omega m, \Omega)=\exp (-\pi \sqrt{-1}\langle\Omega m, m\rangle-2 \pi \sqrt{-1}\langle m, z\rangle) \cdot \theta(z, \Omega), \quad m \in \mathbb{Z}^{n} .
\end{gathered}
$$

Отсюда вытекает предложение 3.1.1. 
3.1.1. $\theta(z, \Omega)$ является единственным (с точностью до умножения на постоянную) сечением расслоения $L$.

Так как $M \rightarrow \widehat{M}$ - изоморфизм, то каждое линейное расслоение на $M$ с $c_{1}=[\omega]$ однозначно представимо в виде $\tau_{\mu}^{*}(L)$ и пространство его глобальных сечений порождается функцией $\theta(z+\mu, \Omega)$.

3.2. Тэта-функции с характеристиками.

Для любой пары векторов $a, b \in \mathbb{R}^{n}$ определяется тәта-функиия с характеристиками а и $b-\theta[a, b](z, \Omega)$ :

$$
\begin{aligned}
& \theta[a, b](z, \Omega)\left(=\theta\left[\begin{array}{l}
a \\
b
\end{array}\right](z, \Omega)\right) \\
& \quad=\exp (\pi \sqrt{-1}\langle\Omega a, a\rangle+2 \pi \sqrt{-1}\langle a, z+b\rangle) \cdot \theta(z+\Omega a+b, \Omega) \\
& \quad=\sum_{m \in \mathbb{Z}^{n}} \exp (\pi \sqrt{-1}\langle\Omega(m+a),(m+a)\rangle+2 \pi \sqrt{-1}\langle m+a, z+b\rangle) .
\end{aligned}
$$

Аналоги условий периодичности (45) для тэта-функций с характеристиками имеют следуюший вид:

$$
\begin{aligned}
\theta[a, b](z+m, \Omega)= & \exp (2 \pi \sqrt{-1}\langle a, m\rangle) \cdot \theta[a, b](z, \Omega), \\
\theta[a, b](z+\Omega m, \Omega)= & \exp (-\pi \sqrt{-1}\langle\Omega m, m\rangle-2 \pi \sqrt{-1}\langle m, z\rangle) \\
& \times \exp (-2 \pi \sqrt{-1}\langle b, m\rangle) \cdot \theta[a, b](z, \Omega), \quad m \in \mathbb{Z}^{n} .
\end{aligned}
$$

Если характеристики $a$ и $b$ рациональны, то функции $\theta[a, b](z)$ порождают сечения линейных расслоений на $M$.

Рассмотрим тензорные степени расслоения $L$. Каждое расслоение $L^{d}$ задается мультипликаторами $e_{\lambda_{j}}^{d}$, где $e_{\lambda_{j}}$ задают $L$ и имеют вид (43).

3.2.1. Любое из семейств функиий

1) $\theta[a / d, 0](d \cdot z, d \cdot \Omega), 0 \leqslant a^{i}<d$,

2) $\theta[0, b / d]\left(z, d^{-1} \cdot \Omega\right), 0 \leqslant b^{i}<d$,

задает базис глобальных сечений расслоения $L^{d}\left(\right.$ m.е. базис в $\left.H^{0}\left(M ; \mathscr{O}\left(L^{d}\right)\right)\right)$.

Эти базисы связаны соотношением

$$
\theta[0, b / d]\left(z, d^{-1} \cdot \Omega\right)=\sum_{a} \exp (2 \pi \sqrt{-1}\langle a, b\rangle) \cdot \theta[a / d, 0](d \cdot z, d \cdot \Omega)
$$

То, что данные функции являются сечениями $L^{d}$, следует из (47). Базисность этих семейств доказывается сравнением их с базисом $\left\{\theta_{\bar{l}}\right\}$ вида (42) сечений $L^{d}$. Соотношение (48) доказьвается сравнением разложений левых и правых частей (48) в ряды $\Phi$ урье.

3.3. Модулярные преобразования.

В $\S 1.4$ было описано действие группы $\operatorname{Sp}\left(\Delta_{\delta}, \mathbb{Z}\right)$ на верхней полуплоскости Зигеля $\mathscr{H}_{n}$. Это действие состоит в замене матрицы $\Omega$ на $\Omega^{\prime}$ такую, что абелевы многообразия $M=\mathbb{C}^{n} /\left\{\Delta_{\delta} \mathbb{Z}^{n}+\Omega \mathbb{Z}^{n}\right\}$ и $\mathbb{C}^{n} /\left\{\Delta_{\delta} \mathbb{Z}^{n}+\Omega^{\prime} \mathbb{Z}^{n}\right\}$ изоморфны. В случае главной 
поляризации $\Delta_{\delta}=I_{n}$ и мы получаем действие $\operatorname{Sp}(2 n, \mathbb{Z})$ :

$$
\begin{aligned}
\Omega \rightarrow \Omega^{\prime} & =(A \Omega+B)(C \Omega+D)^{-1}, \\
z \rightarrow z^{\prime} & =\left((C \Omega+D)^{-1}\right)^{*} \cdot z, \\
g & =\left(\begin{array}{ll}
A & B \\
C & D
\end{array}\right) \in \operatorname{Sp}(2 g, \mathbb{Z}) .
\end{aligned}
$$

3.3.1. При преобразованиях (49) тәта-функция преобразуется следующим образом:

$$
\theta\left[a^{\prime}, b^{\prime}\right]\left(z^{\prime}, \Omega^{\prime}\right)=C_{0} \cdot \exp (\pi \sqrt{-1}\langle z, T z\rangle) \cdot \theta[a, b](z, \Omega),
$$

где постоянная $C_{0}$ не зависит от $z$,

$$
\begin{gathered}
\left(\begin{array}{l}
a^{\prime} \\
b^{\prime}
\end{array}\right)=\left(\begin{array}{cc}
D & -C \\
-B & A
\end{array}\right) \cdot\left(\begin{array}{l}
a \\
b
\end{array}\right)+\frac{1}{2} \cdot\left(\begin{array}{c}
\operatorname{diag}\left(C \cdot D^{*}\right) \\
\operatorname{diag}\left(A \cdot B^{*}\right)
\end{array}\right) \\
T=(C \Omega+D)^{-1} \cdot C .
\end{gathered}
$$

Явньй вид постоянной $C_{0}$ и доказательство формулы (50) приведены в [53].

3.4. Теоремы сложения для тэта-функций.

Наиболее важньми из тэта-функциональных тождеств являются теоремы сложения Римана 3.4.1 и 3.4.2.

3.4.1. БИНАРНАЯ ТЕОРЕМА СЛОЖЕНИЯ РИМАНА.

(51) $\theta[a, c]\left(z_{1}+z_{2}, \Omega\right) \cdot \theta[b, d]\left(z_{1}-z_{2}, \Omega\right)$

$$
=\sum_{e \in \mathbb{Z}^{n} / 2 \mathbb{Z}^{n}} \theta\left[\frac{a+b}{2}+\frac{e}{2}, c+d\right]\left(2 z_{1}, 2 \Omega\right) \cdot \theta\left[\frac{a-b}{2}+\frac{e}{2}, c-d\right]\left(2 z_{2}, 2 \Omega\right)
$$

nри $\theta: \mathbb{C}^{n} \rightarrow \mathbb{C}, z_{1}, z_{2} \in \mathbb{C}^{n}$ u $a, b, c, d \in \mathbb{R}^{n}$.

Формулу (51) достаточно доказать для случая $a=b=c=d=0$, к которому она сводится с помощью (46). В этом случае (51) принимает вид

$$
\theta\left(z_{1}+z_{2}, \Omega\right) \cdot \theta\left(z_{1}-z_{2}, \Omega\right)=\sum_{e \in \mathbb{Z}^{n} / 2 \mathbb{Z}^{n}} \theta\left[\frac{e}{2}, 0\right]\left(2 z_{1}, 2 \Omega\right) \cdot \theta\left[\frac{e}{2}, 0\right]\left(2 z_{2}, 2 \Omega\right) .
$$

Обрашением формулы (52) является следующая теорема сложения:

$$
\theta\left(z_{1}+z_{2}, \Omega\right) \cdot \theta\left(z_{1}-z_{2}, \Omega\right)=\sum_{e \in \mathbb{Z}^{n} / 2 \mathbb{Z}^{n}} \theta\left[0, \frac{e}{2}\right]\left(z_{1}, \frac{\Omega}{2}\right) \cdot \theta\left[0, \frac{e}{2}\right]\left(z_{2}, \frac{\Omega}{2}\right)
$$

Обобщением бинарной теоремы сложения Римана на случай произведения четырех тэта-функций является тернарная теорема сложения. 
3.4.2. ТЕРНАРНАЯ ТЕОРЕМА СЛОЖЕНИЯ РИМАНА.

$$
\begin{aligned}
& \text { (54) } \quad \theta\left[\widetilde{a}_{1}, \widetilde{b}_{1}\right]\left(\widetilde{z}_{1}\right) \cdot \ldots \cdot \theta\left[\widetilde{a}_{4}, \widetilde{b}_{4}\right]\left(\widetilde{z}_{4}\right) \\
& =\frac{1}{2^{n}} \cdot \sum_{c, d \in \frac{1}{2} \mathbb{Z}^{n} / 2 \mathbb{Z}^{n}} \exp \left(-4 \pi \sqrt{-1}\left\langle d, \widetilde{a}_{1}\right\rangle\right) \theta\left[a_{1}+c, b_{1}+d\right]\left(z_{1}\right) \cdot \ldots \cdot \theta\left[a_{4}+c, b_{4}+d\right]\left(z_{4}\right)
\end{aligned}
$$

при $\theta: \mathbb{C}^{n} \rightarrow \mathbb{C}, z_{k} \in \mathbb{C}^{n} u a_{i}, b_{j} \in \mathbb{R}^{n}$, zде $\widetilde{a}=a T, \widetilde{b}=b T, \widetilde{z}=z T u$

$$
T=\frac{1}{2}\left(\begin{array}{rrrr}
1 & 1 & 1 & 1 \\
1 & 1 & -1 & -1 \\
1 & -1 & 1 & -1 \\
1 & -1 & -1 & 1
\end{array}\right)
$$

Здесь единицы в записи матрицы $T$ обозначают единичные $(n \times n)$-матрицы.

Доказательства теорем сложения и их обобшений изложены, например, в [82]. Они получаются прямым сравнением разложений Фурье левых и правых частей (52)-(54) для $n=1$. Для случая общего $n$ надо повторить эти рассуждения покоординатно (см. $[27])$.

\section{5. Тэта-дивизор.}

Дивизором на многообразии $M$ назьвается формальная целочисленная линейная комбинация $D=\sum_{k} a_{k} V_{k}\left(a_{k} \in \mathbb{Z}\right)$ конечного числа аналитических гиперповерхностей в $M$. Дивизоры образуют абелеву группу $\operatorname{Div}(M)$ относительно формального сложения. Дивизор назьвается эффективньм. если $a_{k} \geqslant 0$ для всех $k$.

Дивизором $(f)$ функиии $f$ называется линейная комбинация множеств ее нулей и полюсов, взятых с учетом кратности. При этом множества нулей берутся с положительными, а множества полюсов с отрицательными знаками.

Группа $\operatorname{Div}(M)$ описьвается в терминах сечений пучков следуюшим образом. Пусть $\mathscr{M}^{*}$ - мультипликативньй пучок ростков мероморфных и не равных тождественно нулю функций на $M$. Пучок $\mathscr{O}^{*}$ является подпучком $\mathscr{M}^{*}$. Рассмотрим пространство сечений пучка $\mathscr{M}^{*} / \mathscr{O}^{*}$.

3.5.1. $\operatorname{Div}(M)=H^{0}\left(M ; \mathscr{M}^{*} / \mathscr{O}^{*}\right)$.

Действительно, глобальное сечение пучка $\mathscr{M}^{*} / \mathscr{O}^{*}$ задается как набор локальных сечений $f_{\alpha} \in \mathscr{M}^{*}\left(U_{\alpha}\right)$ таких, что $f_{\alpha} / f_{\beta} \in \mathscr{O}^{*}\left(U_{\alpha} \cap U_{\beta}\right)$. Поэтому локальные дивизоры $\left(f_{\alpha}\right)$ склеиваются в глобальный дивизор, которьй естественно отождествляется с сечением $\left\{f_{\alpha}\right\}$ пучка $\mathscr{M}^{*} / \mathscr{O}^{*}$.

Рассмотрим точную последовательность пучков:

$$
0 \rightarrow \mathscr{O}^{*} \rightarrow \mathscr{M}^{*} \rightarrow \mathscr{M}^{*} / \mathscr{O}^{*} \rightarrow 0
$$

Ей отвечает точная последовательность когомологий, из которой нам интересен лишш следующий фрагмент:

$$
H^{0}\left(M ; \mathscr{M}^{*}\right) \rightarrow H^{0}\left(M ; \mathscr{M}^{*} / \mathscr{O}^{*}\right) \stackrel{\delta}{\rightarrow} H^{1}\left(M ; \mathscr{O}^{*}\right)=\operatorname{Pic}(M) .
$$

Гомоморфизм

$$
\delta: \operatorname{Div}(M) \rightarrow \operatorname{Pic}(M)
$$


сопоставляет дивизору $D$ ассоциированное линейное расслоение следуюшим образом. Пусть дивизор $D$ задается сечением $\left\{f_{\alpha}\right\}$ пучка $\mathscr{M}^{*} / \mathscr{O}^{*}$. Тогда набор функщий $g_{\alpha \beta}=f_{\alpha} / f_{\beta}$ задает функции перехода расслоения $\delta(D)=[D]$.

Дивизоры $D_{1}$ и $D_{2}$ назьваются линейно эквивалентныцми: $D_{1} \approx D_{2}$, если $D_{1}-D_{2} \in \operatorname{Ker} \delta$. Из (56) следует, если $D_{1} \approx D_{2}$, то дивизор $D_{1}-D_{2}$ является дивизором мероморфной функции на $M$.

Тәта-дивизором $\Theta=(\theta)$ называется множество нулей тэта-функции (44).

Следующее утверждение очевидно.

3.5.2. 1) Линейное расслоение $L=[\Theta]$ задается мультипликаторами (43), и тәта-функиия порождает $H^{0}(M ; \mathscr{O}(L))$.

2) $[\Theta+\mu]=\tau_{-\mu}^{*}(L)$.

Для полноты изложения мы приведем следуюший факт, доказательство которого основано на теореме Стокса и изложено, например, в [41].

3.5.3. Пусть $M$ - компактное комплексное $n$-мерное многообразие, $L-л и$ нейное расслоение на $M$ и $L=[D]$. Тогда $c_{1}(L)$ двойственен (по Пуанкаре) ииклу $D \in H_{2 n-2}(M ; \mathbb{Z})$.

\section{$\S 4$. Тэта-функции и отображения торов в проективные пространства. Секущие абелевых многообразий}

\section{1. Линейные системы.}

Пусть $L=[D]$ - линейное расслоение на компактном комплексном многообразии, ассоциированное с эффективным дивизором $D$. Локально дивизор $D$ задан функциями $f_{\alpha} \in \mathscr{M}\left(U_{\alpha}\right)$, где $\mathscr{M}$ - пучок ростков мероморфных сечений $L$. Эти функции задают, как можно заметить, мероморфное сечение $f_{0}$ расслоения $L$.

Обозначим через $\mathscr{L}(D)$ пространство таких мероморфных функций $f$ на $M$, что дивизор $(f)+D$ эффективен $((f)+D \geqslant 0)$. Через $|D|$ обозначим множество эффективных дивизоров, линейно эквивалентных $D$. Существует взаимно однозначное соответствие между точками проективизации пространства $\mathscr{L}(D)$ и дивизорами из $|D|$ :

$$
D^{\prime} \in|D| \longleftrightarrow[f] \in P \mathscr{L}(D):(f)=D^{\prime}-D .
$$

Сечение $f \cdot f_{0}$ пучка $\mathscr{M}$ голоморфно, если и только если $f \in \mathscr{L}(D)$. Поэтому (58) продолжается до соответствия

$$
|D| \longleftrightarrow P H^{0}(M ; \mathscr{O}([D])) .
$$

Множество эффективных дивизоров, отвечающее при соответствии (59) линейному подпространству $H^{0}(M ; \mathscr{O}([D]))$, называется линейной системой дивизоров. Простейшим примером является полная линейная система $|D|$.

Базисныцм множеством $B(X)$ линейной системы $X$ назьвается пересечение всех дивизоров этой системы. Если $E(X) \subset H^{0}(M ; \mathcal{O}([D]))$ - подпространство, отвечающее $X$ при (59), то $B(X)$ состоит из точек $M$, в которых все сечения из $E(X)$ равны нулю. 
Для каждой линейной системы $X$ определено (с точностью до проективных преобразований $\mathbb{C P}^{k}$ ) отображение

$$
\varphi: M \backslash B(X) \rightarrow \mathbb{C P}^{k}: \varphi(x)=\left(f_{1}(x): \cdots: f_{k+1}(x)\right)
$$

где $\left\{f_{j}\right\}-$ базис в $E(X)$.

4.2. Линейные системы $|d \Theta|$. Теорема Лефшеца.

Рассмотрим отображения (60) для линейных систем $|d \Theta|, d \geqslant 2$.

4.2.1. При $d \geqslant 2$ базисные множества систем $|d \Theta|$ пусты.

Для доказательства этого предложения достаточно для любой точки $z \in M=$ $\mathbb{C}^{n} /\left\{\mathbb{Z}^{n}+\Omega \mathbb{Z}^{n}\right\}$ найти сечения $f_{d} \in H^{0}\left(M ; \mathscr{O}\left(L^{d}\right)\right)$ такие, что $f_{d}(z) \neq 0$.

Согласно (45) функция $f_{d}^{\mu}(z)=\theta\left(z+\mu_{1}\right) \cdot \ldots \cdot \theta\left(z+\mu_{d}\right)$ задает глобальное сечение $\mathscr{O}\left(L^{d}\right)$ при $\mu_{1}+\cdots+\mu_{d}=0$. Так как тэта-дивизор является аналитической гиперповерхностью в $M$, то для любой точки $z \in M$ существует вектор $\nu=\left(\nu_{1}, \ldots, \nu_{d}\right)$ такой, что $\theta\left(z+\nu_{k}\right) \neq 0$ при любом $k$. Теперь в качестве $f_{d}$ достаточно взять $f_{d}^{\nu}$. Предложение 4.2.1 доказано.

Обозначим через

$$
\varphi_{d}: M=\mathbb{C}^{n} /\left\{\mathbb{Z}^{n}+\Omega \mathbb{Z}^{n}\right\} \rightarrow \mathbb{C P}^{d^{n}-1}
$$

отображение (60) для линейной системы $|d \Theta|$. Согласно предложению 3.2.1 отображение $\varphi_{d}$ может быть задано формулами

$$
\varphi_{d}(z)=\left(\theta\left[\frac{a_{1}}{d}, 0\right](d \cdot z, d \cdot \Omega): \cdots: \theta\left[\frac{a_{d^{n}}}{d}, 0\right](d \cdot z, d \cdot \Omega)\right) \in \mathbb{C P}^{d^{n}-1}
$$

где $\left\{a_{i}\right\}=\mathbb{Z}^{n} / d \mathbb{Z}^{n}$.

4.2.2. ТЕОРЕМА ЛЕФШЕЦА. При $d \geqslant 3$ отображение $\varphi_{d}$ является вложсением.

Мы, ради краткости, дадим доказательство лишш при $d=3$. Из доказательства предложения 4.2.1 ясно, как получается общий случай.

1) Прежде всего докажем, что ранг $\varphi_{d}$ максимален.

Пусть $z_{*} \in M$ - произвольная точка тора. Выберем базис $\left(\theta_{0}, \theta_{1}, \ldots, \theta_{N}\right)$ в $H^{0}\left(M ; \mathscr{O}\left(L^{2}\right)\right)$ таким, что $\theta_{0}\left(z_{*}\right)=1$ и $\theta_{1}\left(z_{*}\right)=\cdots=\theta_{N}\left(z_{*}\right)=0$. Тогда ранг отображения $\varphi_{2}$ в точке $z_{*}$ совпадает с рангом матрищы

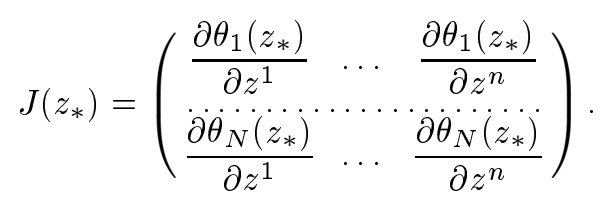

Предположим, что $\operatorname{rank} J\left(z_{*}\right)<n$. Это означает, что существует нетривиальньй набор постоянных $a_{1}, \ldots, a_{n}$ такой, что

$$
a_{1} \frac{\partial \theta_{k}\left(z_{*}\right)}{\partial z^{1}}+\cdots+a_{n} \frac{\partial \theta_{k}\left(z_{*}\right)}{\partial z^{n}}=0
$$


для любого $k$. Выберем такие $\mu$ и $\nu$, что

$$
\theta\left(z_{*}+\nu\right) \cdot \theta\left(z_{*}-\mu-\nu\right) \neq 0
$$

Из (45) следует, что функция

$$
F(z, \mu, \nu)=\theta(z+\mu) \cdot \theta(z+\nu) \cdot \theta(z-\mu-\nu)
$$

задает сечение $L^{d}(d=3)$ при любых $\mu, \nu$. Значит, она раскладьвается по базису $\left\{\theta_{k}\right\}$ и для нее вьполнено соотношение

$$
a_{1} \frac{\partial F\left(z_{*}, \mu, \nu\right)}{\partial z^{1}}+\cdots+a_{n} \frac{\partial F\left(z_{*}, \mu, \nu\right)}{\partial z^{n}}=0
$$

Определим функцию

$$
\xi(z)=a_{1} \frac{\partial \log \theta(z)}{\partial z^{1}}+\cdots+a_{n} \frac{\partial \log \theta(z)}{\partial z^{n}}
$$

Можно заметить, что (65) эквивалентно следующему равенству:

$$
\xi(z+\mu)+\xi(z+\nu)+\xi(z-\mu-\nu)=0 .
$$

Из (63) и (66) следует, что функция $\widetilde{\xi}(z)=\xi(z+\mu)$ не имеет полюсов и потому является целой. Согласно (25) функция $\widetilde{\xi}(z)$ удовлетворяет следуюшим условиям периодичности:

$$
\widetilde{\xi}\left(z+\lambda_{k}\right)=\widetilde{\xi}(z), \quad \widetilde{\xi}\left(z+\lambda_{n+k}\right)=\widetilde{\xi}(z)-2 \pi \sqrt{-1} a_{k}
$$

Согласно (67) производные $\partial \widetilde{\xi} / \partial z^{k}$ функции $\widetilde{\xi}$ периодичны относительно $\Lambda$ и потому ограничены. Так как они являются цельми функциями, то это означает, что они постоянны и $\widetilde{\xi}(z)=\sum_{k} b_{k} z^{k}+c$ линейна. Но из (67) следует, что $b_{k} \equiv 0$ и, значит, функция $\widetilde{\xi}$ постоянна. Так как $\widetilde{\xi}\left(z+\lambda_{n+k}\right)-\widetilde{\xi}(z)=-2 \pi \sqrt{-1} a_{k}$, то $a_{k} \equiv 0$.

Мы заключаем, что набор постоянных $\left\{a_{k}\right\}$ тривиален и матрица $J\left(z_{*}\right)$ имеет максимальный ранг. Так как точка $z_{*}$ была выбрана произвольной, то ранг $\varphi_{d}$ всюду равен $n$.

2) Докажем, что из $\varphi_{d}\left(z_{1}\right)=\varphi_{d}\left(z_{2}\right)$ следует, что $z_{1}-z_{2} \in \Lambda$. Это эквивалентно инъективности $\varphi_{d}$.

Пусть $\varphi_{d}\left(z_{1}\right)=\varphi_{d}\left(z_{2}\right)$. Значит, существует ненулевая постоянная $C$ такая, что $\psi\left(z_{1}\right)=C \psi\left(z_{2}\right)$ для любого сечения $\psi$ расслоения $L^{d}$. Рассмотрим функцию $F(z, \mu, \nu)$ вида (64). Так как она задает сечение $L^{d}$, то

$$
\frac{F\left(z_{1}, \mu, \nu\right)}{F\left(z_{2}, \mu, \nu\right)}=\frac{\theta\left(z_{1}+\mu\right) \cdot \theta\left(z_{1}+\nu\right) \cdot \theta\left(z_{1}-\mu-\nu\right)}{\theta\left(z_{2}+\mu\right) \cdot \theta\left(z_{2}+\nu\right) \cdot \theta\left(z_{2}-\mu-\nu\right)}=C .
$$

Для любого $\mu$ можно так подобрать $\nu$, что

$$
\theta\left(z_{1}+\nu\right) \cdot \theta\left(z_{1}-\mu-\nu\right) \cdot \theta\left(z_{2}+\nu\right) \cdot \theta\left(z_{2}-\mu-\nu\right) \neq 0
$$


Из (68), (69) следует, что функция $\widehat{\xi}=\log \left(\theta\left(z_{1}+z\right) / \theta\left(z_{2}+z\right)\right)$ голоморфна. Как и для функции $\widetilde{\xi}$, можно показать, что производные $\widehat{\xi}(z)$ постоянны и потому эта функция линейна: $\widehat{\xi}(z)=2 \pi \sqrt{-1} \sum b_{k} z^{k}+c$. Из (45) следует, что

$$
\begin{gathered}
\widehat{\xi}\left(z+\lambda_{k}\right)=\widehat{\xi}(z)+2 \pi \sqrt{-1} \beta_{k}, \\
\widehat{\xi}\left(z+\lambda_{n+k}\right)=\widehat{\xi}(z)+2 \pi \sqrt{-1}\left(z_{2}^{k}-z_{1}^{k}\right)+2 \pi \sqrt{-1} \alpha_{k}, \quad \alpha_{i}, \beta_{j} \in \mathbb{Z} .
\end{gathered}
$$

Так как $\widehat{\xi}$ линейна, то $b_{k}=\beta_{k}$ и

$$
z_{2}^{k}-z_{1}^{k}=-\alpha_{k}+\sum_{l} \Omega_{k l} \beta_{l}
$$

Последнее равенство эквивалентно тому, что $z_{2}-z_{1} \in \Lambda$. Теорема Леффшеца доказана.

Из теорем Лефшеца и Чжоу вытекает, что

4.2.3. Главно поляризованный ходжев тор является абелевым многообразиe⿻从.

В случае, когда ходжев тор $(M,[\omega])$ не главно поляризован, то пространство сечений линейного расслоения $L \rightarrow M$ с $c_{1}(L)=[\omega]$ порождается функциями $\theta_{\bar{l}}$ вида (40)-(42). Заменив в доказательстве предложения 4.2.1 и теоремы Лефшеца тэта-функцию $\theta(z, \Omega)$ на любую из функций $\theta_{\bar{l}}$, мы можем повторить все рассуждения и доказать следуюшее утверждение.

4.2.4. Теорема ЛЕфшецА (общий случай). Пусть тор $(M,[\omega])$ ходжев, $L$ - линейное расслоение с $c_{1}(L)=[\omega]$ и $X_{d}$ - линейная система, отвечающая $H^{0}\left(M ; \mathscr{O}\left(L^{d}\right)\right)$. Тогда ее базисное множество пусто при $d \geqslant 2$, и она задает посредством (60) вложение тора $M$ в комплексное проективное пространство npu $d \geqslant 3$.

Отсюда и из теоремы Чжоу следует

4.2.5. КРИТЕРИЙ АЛГЕБРАИЧНОСТИ КОМПЛЕКСНЫХ ТОРОВ. Комплексный тор является абелевым многообразием тогда и только тогда, когда он ходжев.

В общем случае доказательство теоремы Кодаиры тоже состоит в том, что по ходжевой форме $\omega$ строится линейное расслоение $L$ с $c_{1}(L)=[\omega]$, и отображение $M$ в $\mathbb{C P}^{N}$ имеет вид (60) для линейной системы, отвечающей $H^{0}\left(M ; \mathscr{O}\left(L^{d}\right)\right)$ при достаточно большом $d$ (см. [17], [41]).

4.3. Отображение $\varphi_{2}$ и многообразия Куммера.

Пусть $M=\mathbb{C}^{n} / \Lambda$ - абелево многообразие с главной поляризацией $[\omega]$. На нем действует инволюция (отражение)

$$
\sigma: M \rightarrow M: \sigma(z)=-z
$$

Эта инволюция объясняет, почему утверждение теоремы Лефшеца не верно при $d=2$. Отображение $\varphi_{2}$ порождено сечениями $\theta[e, 0](d \cdot z, 2 \Omega)\left(2 e \in \mathbb{Z}^{n} / 2 \mathbb{Z}^{n}\right)$ расслоения $[2 \Theta]$. 
Все эти функции четны, и ранг отображения $\varphi_{2}$ в точке $z=0$ равен нулю. Это также означает, что $\varphi_{2}$ разлагается в композицию

$$
M \stackrel{\pi}{\longrightarrow} M / \sigma \stackrel{\Phi}{\longrightarrow} \mathbb{C P}^{2^{n}-1}, \quad \varphi_{2}=\Phi \circ \pi
$$

где $\pi$ - естественная проекция.

Многообразие $K(M, \Theta)=\Phi(M / \sigma)$ назьвается многообразием Куммера, а отображение $\Phi$ - отображением Куммера.

Поляризованное абелево многообразие $(M,[\omega])$ назьвается разложимым, если оно представимо в виде прямого произведения абелевых многообразий $\left(M_{1},\left[\omega_{1}\right]\right)$ и $\left(M_{2},\left[\omega_{2}\right]\right)$ положительной размерности и $[\omega]=\left[\omega_{1}\right]+\left[\omega_{2}\right]$.

4.3.1. ТЕОРЕМА ОБ ИНЪЕКТИВНОСТИ ОТОБРАЖЕНИЯ КУММЕРА. Если главно поляризованное абелево многообразие $M=\mathbb{C}^{g} /\left\{\mathbb{Z}^{g}+\Omega \mathbb{Z}^{g}\right\}$ неразложимо, то отображение Куммера

$$
\Phi: z \rightarrow\left(\theta\left[n_{1}, 0\right](2 z, 2 \Omega): \ldots: \theta\left[n_{r}, 0\right](2 z, 2 \Omega)\right)
$$

$\left(\left\{2 n_{j}\right\}=\mathbb{Z}^{g} / 2 \mathbb{Z}^{g}, r=2^{g}\right)$ является влоэсением многообразия (с особенностями) $M / \sigma \quad \boldsymbol{\theta} \mathbb{C P}^{2^{n}-1}$.

Мы изложим доказательство этой теоремы по модулю предложения 4.3.2.

4.3.2. Если абелево многообразие главно поляризовано и неразложимо, то его тэта-дивизор $\Theta$ неприводим и не инвариантен относительно сдвигов (т.е. если $\Theta+\mu=\Theta$, то $\mu \in \Lambda$ ).

В целях краткости мы опустим доказательство этого предложения. Заметим, что оно основано на том, что разложимость главно поляризованного абелева многообразия эквивалентна приводимости тэта-дивизора, а наличие нетривиальных сдвигов, переводяших многообразие само в себя, несовместимо с главной поляризацией.

Пусть $\varphi_{2}\left(z_{1}\right)=\varphi_{2}\left(z_{2}\right)$. Тогда существует ненулевая постоянная $C$ такая, что

$$
\frac{\theta[e, 0]\left(2 z_{1}, 2 \Omega\right)}{\theta[e, 0]\left(2 z_{2}, 2 \Omega\right)}=C
$$

для всех $e \in \frac{1}{2} \mathbb{Z}^{n} / 2 \mathbb{Z}^{n}$. Из теоремы сложения (52) следует, что

$$
\frac{\theta\left(z+z_{1}\right) \cdot \theta\left(z-z_{1}\right)}{\theta\left(z+z_{2}\right) \cdot \theta\left(z-z_{2}\right)}=C
$$

для любого $z \in \mathbb{C}^{n}$.

Пусть $\left(z+z_{1}\right)$-неособая точка тэта-дивизора и $\theta\left(z-z_{1}\right) \neq 0$. Тогда из $(71)$ следует, что $\theta\left(z+z_{2}\right) \cdot \theta\left(z-z_{2}\right)=0$.

Предположим, что $\theta\left(z-z_{2}\right)=0$. Тогда в окрестности точки $z$ функщия $\theta\left(z+z_{1}\right) / \theta\left(z-z_{2}\right)$ голоморфна и, так как тэта-дивизор неприводим, то $\Theta+\left(z_{1}+z_{2}\right)=\Theta$. Так как тэта-дивизор не инвариантен относительно сдвигов, то $z_{1}+z_{2} \in \Lambda$, что и требовалось доказать. Случай $\theta\left(z+z_{2}\right)=0$ разбирается аналогично. Теорема доказана. 
Важньм примером многообразия Куммера является фактор-пространство двумерного комплексного тора $\mathbb{C}^{2} / \Lambda$ по отражению. Оно имеет 16 особых точек, отвечающих подрешетке $\Lambda / 2$. После разрешения особенностей мы получаем неособую алгебраическую поверхность, известную как поверхность Куммера или $K 3$ поверхность.

4.4. Секущие абелевых многообразий.

Пусть $M=\mathbb{C}^{n} / \Lambda$ - неразложимое главно поляризованное абелево многообразие и $K(M, \Theta) \subset \mathbb{C P}^{2^{n}-1}$ - его многообразие Куммера.

ОПРЕДЕЛЕНИЕ. $N$-секущей абелева многообразия $M$ назьвается $(N-2)$-мерная плоскость $V\left(=\mathbb{C P}^{N-2}\right) \subset \mathbb{C P}^{2^{n}-1}$, пересекающая многообразие Куммера $K(M, \Theta)$ по $N$ различным точкам.

Пусть $V-N$-секущая, проходящая через точки $\Phi\left(z_{1}\right), \ldots, \Phi\left(z_{N}\right)$. Тогда существуют такие ненулевые постоянные $c_{1}, \ldots, c_{N}$, что

$$
c_{1} \cdot \theta[e, 0]\left(2 z_{1}, 2 \Omega\right)+\cdots+c_{N} \cdot \theta[e, 0]\left(2 z_{N}, 2 \Omega\right)=0
$$

для любого $e \in \frac{1}{2} \mathbb{Z}^{n} / 2 \mathbb{Z}^{n}$. Из теоремы сложения (52) и (72) следует

4.4.1. Утверждения (i) и (ii) әквивалентны:

(i) точки $\Phi\left(z_{1}\right), \ldots, \Phi\left(z_{N}\right)$ многообразия Kуммера $K(M, \Theta)$ лежсат на $N$-плоскости, удовлетворяя соотношению $c_{1} \Phi\left(z_{1}\right)+\cdots+c_{N} \Phi\left(z_{N}\right)=0$,

(ii) выполняется тэта-функциональное соотношение

$$
c_{1} \theta\left(z+z_{1}\right) \theta\left(z-z_{1}\right)+\cdots+c_{N} \theta\left(z+z_{N}\right) \theta\left(z-z_{N}\right)=0, \quad z \in \mathbb{C}^{n} .
$$

Рассмотрим условия на тэта-дивизор, которые обеспечивают наличие $N$-секущих . По-видимому, впервые они рассматривались в [111]. Через $\Theta_{\mu}$ обозначим тэта-дивизор, сдвинутьй на $\mu$, т.е. $\Theta+\mu$.

4.4.2. Пусть $\operatorname{dim} M \geqslant(N-1)$. Утверждения (i) и (iі) әквивалентны:

(i) существуют такие различные точки $\mu_{1}, \ldots, \mu_{N-1}, x, y \in M$, что аналитическое подмножество $\Theta_{\mu_{1}} \cap \cdots \cap \Theta_{\mu_{N-1}}$ имеет коразмерность $N-1$ $u$

$$
\Theta_{\mu_{1}} \cap \cdots \cap \Theta_{\mu_{N-1}} \subset \Theta_{x} \cup \Theta_{y}
$$

(ii) многообразие Куммера $K(M, \Theta)$ имеет $N$-секущую и выполняется (73) для $z_{1}=(x+y) / 2-\mu_{1}, \ldots, z_{N-1}=(x+y) / 2-\mu_{N-1}, z_{N}=(x-y) / 2 u$ $c_{N} \neq 0$.

То, что (ii) влечет за собой (i), следует из предложения 4.4.1. Доказательство того, что (i) влечет за собой (ii), использует комплекс Кошуля пересечения $\Theta_{\mu_{1}} \cap \cdots \cap \Theta_{\mu_{N-1}}$ (см. [49]). Мы ограничимся случаем $N=4$ (см. [7]), из которого ясно, как доказательство усложняется при $N>4$ и упрошается при $N=3$ (см. [82]).

Рассмотрим расслоение $L=[2 \Theta+x+y]$ и его сечения $s_{0}=\theta(z-x) \theta(z-y)$, $s_{1}=\theta\left(z-\mu_{1}\right) \theta\left(z-\left(x+y-\mu_{1}\right)\right), \ldots, s_{3}=\theta\left(z-\mu_{3}\right) \theta\left(z-\left(x+y-\mu_{3}\right)\right)$. Через $I$ обозначим подпучок $\mathscr{O}_{M}$, чьи сечения обращаются в нуль на $\Theta_{\mu_{1}} \cap \cdots \cap \Theta_{\mu_{N-3}}$. 
Рассмотрим комплекс Кошуля $\left(K_{i}, d_{i}\right)$, тензорно умноженньй на $\mathscr{O}(L)$,

$$
0 \longrightarrow \mathscr{O}\left(L^{-2}\right) \stackrel{d_{1}}{\longrightarrow} \mathscr{O}\left(L^{-1}\right) \oplus \mathscr{O}\left(L^{-1}\right) \oplus \mathscr{O}\left(L^{-1}\right) \stackrel{d_{2}}{\longrightarrow} \mathscr{O} \oplus \mathscr{O} \oplus \mathscr{O} \stackrel{d_{3}}{\longrightarrow} I \otimes \mathscr{O}(L) \longrightarrow 0,
$$

где $d_{1}(f)=\left(s_{1} f, s_{2} f, s_{3} f\right), d_{2}(f, g, h)=\left(c_{2} h-c_{3} g, c_{3} f-c_{1} h, c_{1} g-c_{2} f\right), d_{3}(f, g, h)=$ $\left(s_{1} f+s_{2} g+s_{3} h\right)$. Эта последовательность точна, так как $\operatorname{dim} \Theta_{\mu_{1}} \cap \Theta_{\mu_{2}} \cap \Theta_{\mu_{3}}=$ $\operatorname{dim} M-3$.

Согласно теореме Кодаиры об обрашении в нуль, примененной к положительному расслоению $L$ (см. [17], [41]), $H^{k}\left(M ; \mathscr{O}\left(L^{-m}\right)=0\right.$ при $m>0$ и $k<\operatorname{dim} M$. Последовательно применяя точную когомологическую последовательность к тройкам $0 \rightarrow A=K_{i} / d_{i-1}\left(K_{i-1}\right) \rightarrow K_{i+1} \rightarrow K_{i+1} / d_{i+1}(A) \rightarrow 0$, мы придем к точной последовательности

$$
0 \longrightarrow \widetilde{A} \stackrel{d_{N-2}}{\longrightarrow} \mathscr{O} \oplus \cdots \oplus \mathscr{O} \stackrel{d_{N-1}}{\longrightarrow} I \cdot \mathscr{O}(L),
$$

где $H^{1}(M ; \widetilde{A})=0$. Из точной когомологической последовательности для $(75)$ следует, что

$$
H^{0}(M ; I \otimes \mathscr{O}(L))=d_{N-1}\left(H^{0}(M ; \mathscr{O} \oplus \cdots \oplus \mathscr{O})\right)=d_{N-1}\left(\mathbb{C}^{N-1}\right) .
$$

Функция $s_{0}$ задает согласно $(74)$ сечение $I \otimes \mathscr{O}(L)$ и потому является линейной комбинацией сечений $s_{1}, \ldots, s_{N-1}$. Это согласно (73) эквивалентно существованию секущей. Предложение 4.4.2 доказано.

\section{ГЛАВА ІІ
ТЭТА-ФУНКЦИИ РИМАНОВЫХ ПОВЕРХНОСТЕЙ}

\section{§. Тэта-функции многообразий Якоби}

\section{1. Римановы поверхности и их матрицы периодов.}

Римановой поверхностью назьвается комплексное многообразие размерности (комплексной) один. Если риманова поверхность Г компактна, то она гомеоморфна двумерной сфере с ручками, число которых назьвается родом Г.

Базис $a_{1}, \ldots, a_{g}, b_{1}, \ldots, b_{g} 1$-циклов на компактной поверхности Г рода $g$ назьвается каноническим, если его форма пересечения имеет вид

$$
a_{i} \circ a_{j}=b_{i} \circ b_{j}=0, \quad a_{i} \circ b_{j}=\delta_{i j}
$$

где $\delta_{i j}$ - символ Кронекера. Канонический базис реализуется такими контурами, что после разрезания по ним поверхность Г преврашается в $4 g$-угольник $\widetilde{\Gamma}$ со сторонами (взятыми в порядке обхода) $a_{1}, b_{1}, a_{1}^{-1}, b_{1}^{-1}, \ldots, a_{g}, b_{g}, a_{g}^{-1}, b_{g}^{-1}$.

Всюду в дальнейшем римановы поверхности подразумеваются компактными, а базисы 1-циклов на них - каноническими.

Так как эрмитова метрика на одномерном комплексном многообразии всегда пропорциональна ходжевой, то любая риманова поверхность проективно алгебраична (является алгебраической кривой). 
Дифференциалом называется 1-форма $\omega$ на Г. Дифференциал называется абелевыц (или голоморфньм), если в окрестности любой точки он записьвается в виде $\omega=f(z) d z$, где $f(z)$ - голоморфная функция. Аналогично определяются мероморфные дифференциалы. Очевидно, что абелев дифференщиал всегда замкнут, как и сопряженньй к нему $\bar{\omega}=\bar{f}(z) d \bar{z}$.

5.1.1. ЗАКОн ВЗАимности (для гладких дифференщиалов). Пусть $\omega u \omega^{\prime}-$ замкнутые дифференциаль на римановой поверхности рода $g$. Тогда

$$
\int_{\Gamma} \omega \wedge \omega^{\prime}=\sum_{k=1}^{g}\left(\int_{a_{k}} \omega \cdot \int_{b_{k}} \omega^{\prime}-\int_{b_{k}} \omega \cdot \int_{a_{k}} \omega^{\prime}\right) .
$$

Доказательство этого предложения состоит в переходе к универсальной накрьвающей $\{z \in \mathbb{C}: \operatorname{Im} z>0\} \rightarrow \Gamma$ римановой поверхности и применении теоремы Стокса к области $\widetilde{\Gamma}$, которая проектируется на $\Gamma$. Применяя его к абелеву дифференциалу $\omega$ и его сопряженному $\bar{\omega}$, получаем

5.1.2. Если $\omega$ - ненулевой голоморфный дифференциал на римановой поверхности рода $g$, то

$$
\operatorname{Im} \sum_{k=1}^{g} \int_{a_{k}} \omega \cdot \int_{b_{k}} \omega<0 .
$$

Сопряжение $\omega \rightarrow \bar{\omega}$ индуцирует инволюцию на группе $H^{1}(M ; \mathbb{C})$, которая распадается в прямую сумму подгрупा $H^{1,0}(M ; \mathbb{C})$ и $H^{0,1}(M ; \mathbb{C})$, порожденных голоморфными и антиголоморфными дифференциалами. Эти подгрупшы переставляются сопряжением и изоморфны $\mathbb{C}^{g}$. Отсюда и из $(77)$ следует

5.1.3. Пространство голоморфных дифференциалов на римановой поверхности рода g изоморфно $\mathbb{C}^{g}$, и каждый голоморфный дифференциал однозначно определяется своими а-периодами.

Поэтому каждому каноническому базису 1-циклов сопоставляется канонический базис абелевых дифференциалов $\omega_{1}, \ldots, \omega_{g}$, однозначно определенньй условием

$$
\int_{a_{j}} \omega_{k}=\delta_{j k}
$$

По этому базису строится матрица периодов римановой поверхности Г:

$$
B(\Gamma)_{j k}=\int_{b_{j}} \omega_{k}
$$

Следовательно, матрица периодов зависит от выбора канонического базиса в $H_{1}(\Gamma)$. Переход к новому каноническому базису задается преобразованием из $\mathrm{Sp}(2 g, \mathbb{Z})$. Матрица периодов при этом преобразуется согласно (16). Применяя (77) к дифференщиалам вида $\omega=\sum_{k} c_{k} \omega_{k}, c_{k} \in \mathbb{R}$, получаем 
5.1.4. Матрица периодов римановой поверхности принадлежит верхней полуплоскости Зигеля: $B(\Gamma) \in \mathscr{H}_{g}$.

Римановы поверхности рода $g$ находятся во взаимно однозначном соответствии с точками комплексного многообразия $\mathscr{M}_{g}$ - пространства модулей римановых поверхностей рода $g$. Известно, что

$$
\operatorname{dim}_{\mathbb{C}} \mathscr{M}_{g}= \begin{cases}0, & g=0 \\ 1, & g=1 \\ 3 g-3, & g \geqslant 2\end{cases}
$$

Значит, определено отображение

$$
B: \mathscr{M}_{g} \rightarrow \mathscr{A}_{g}=\mathscr{H}_{g} / \operatorname{Sp}(2 g, \mathbb{Z})
$$

После надлежашей компактификации пространства $\mathscr{M}_{g}$ отображение $B$ становится алгебраическим, как и определенные ниже отображения Прима (99) и (116) (см. [85]).

5.2. Многообразие Якоби и отображение Абеля. Тэта-функция римановой поверхности.

Из (81) следует, что римановой поверхности Г канонически сопоставляется главно поляризованное абелево многообразие - многообразие Якоби:

$$
J(\Gamma)=\mathbb{C}^{g} /\left\{\mathbb{Z}^{g}+B(\Gamma) \mathbb{Z}^{g}\right\}
$$

Зафиксируем точку $p_{0} \in \Gamma$ и рассмотрим отображение

$$
A: \Gamma \rightarrow J(\Gamma): A(p)=\left(\int_{p_{0}}^{p} \omega_{1}, \ldots, \int_{p_{0}}^{p} \omega_{g}\right)
$$

которое называется отображением Абеля. Его определение не зависит от выбора пути интегрирования $\gamma$. Действительно, заменим путь $\gamma$ на $\gamma^{\prime}$. Контур $\gamma^{-1} \cdot \gamma^{\prime}$ замкнут и реализует 1-цикл $\zeta$. Значение $A(p)$ изменится на вектор $\left(\int_{\zeta} \omega_{1}, \ldots, \int_{\zeta} \omega_{g}\right)$, которьй принадлежит решетке $\left\{\mathbb{Z}^{g}+B(\Gamma) \mathbb{Z}^{g}\right\}$.

Отображение Абеля линейно продолжается на всю группу дивизоров на $\Gamma$.

Пусть $\theta(z)=\theta(z, B(\Gamma))-$ тәта-функиия римановой поверхности Г. Построим по ней функции вида $f(p)=\theta(A(p)-e)$. Эти функции многозначны на $\Gamma$, так как их значения зависят от выбора пути интегрирования в (83). Разница между ветвями определяется формулами (45). Но из (45) видно, что нули функщии $f(p)$ образуют корректно определенньй дивизор на $\Gamma$, если $f$ не обрашается тождественно в нуль.

5.2.1. Существует такая точка $K_{\Gamma} \in J(\Gamma)$ ( "вектор римановых констант"), что выполняется одна из двух возможнностей:

1) $A(\Gamma) \subset \Theta_{e}=\Theta+e u \theta(A(p)-e) \equiv 0$;

2) ecлu $f(p)=\theta(A(p)-e)=0$, mo $p \in\left\{p_{1}, \ldots, p_{g}\right\} u$

$$
A\left(p_{1}\right)+\cdots+A\left(p_{g}\right)+K_{\Gamma}=e .
$$


В случае 2) дивизор $D=p_{1}+\cdots+p_{g}$ определяется по (84) однозначно.

Так как случай 2) выполняется для почти всех $e \in J(\Gamma)$, то формула (84) задает обращение отображения

$$
A: S^{g} \Gamma \rightarrow J(\Gamma), \quad A\left(p_{1}, \ldots, p_{g}\right)=A\left(p_{1}\right)+\cdots+A\left(p_{g}\right),
$$

где $S^{g} \Gamma-g$-я симметрическая степень поверхности $Г$, почти всюду.

Если эффективный дивизор $D$ степени $g$ является дивизором полюсов нетривиальной мероморфной функции, то в точке $A(D)$ отображение $A$ очевидно необратимо. Такие дивизоры называются специальнымми. Более того,

5.2.2. Пусть $\Sigma \subset S^{g}$ - подмножество, образованное специальнымми дивизорами. Тогда на множестве $S^{g} \backslash \Sigma$ отображение $A$ обратимо посредством (84).

Тэта-дивизор $\Theta$ описывается следующей теоремой.

\subsection{3. ТЕОРЕМА РИМАНА О ТЭТА-ДИВИЗОРе МНОГООБРАЗИЯ ЯКОБИ.}

$$
\Theta=A\left(S^{g-1}\right)+K_{\Gamma} .
$$

\section{3. Теоремы Абеля и Римана-Роха.}

Дивизорами на поверхности являются линейные комбинации конечного числа ее точек вида $D=a_{1} p_{1}+\cdots+a_{n} p_{n}, a_{k} \in \mathbb{Z}, p_{k} \in \Gamma$. Степенью дивизора $D$ назьвается следующая сумма: $\operatorname{deg} D=a_{1}+\cdots+a_{n} \in \mathbb{Z}$.

По каждому дивизору $D$ строится линейное расслоение $[D]=\delta(D)($ см. $\S 3.5,(57))$. Доказательство следующего утверждения изложено в [48].

5.3.1. Если $M$ - проективное алгебраическое многообразие, то любое линейное расслоение на $M$ имеет мероморфное сечение, и потому образ гомоморфизма (57) $\delta: \operatorname{Div}(M) \rightarrow \operatorname{Pic}(M)$ совпадает $c \operatorname{Pic}(M)$.

Теорема Абеля позволяет линеаризовать $\delta$.

5.3.2. ТЕОрема АБЕля. Пусть $\operatorname{deg} D_{1}=\operatorname{deg} D_{2}$. Дивизор $D_{1}-D_{2}$ является дивизором мероморфной функиии на Г тогда и только тогда, когда $A\left(D_{1}\right)=$ $A\left(D_{2}\right)$.

Из точной последовательности (56), предложения 5.3.1 и теоремы Абеля следует

\subsection{3. СТрУКтУРА ГРУПпы $\operatorname{Pic}(Г)$. Последовательность}

$$
0 \rightarrow J(\Gamma) \rightarrow \operatorname{Pic}(\Gamma) \stackrel{\operatorname{deg}}{\longrightarrow} \mathbb{Z} \rightarrow 0
$$

точна.

В (86) первый гомоморфизм является вложением подгруппы, порожденной расслоениями $[D]$ с $\operatorname{deg} D=0$. Эта последовательность аналогична (35). Действительно, $H^{2}(\Gamma ; \mathbb{Z})=\mathbb{Z}$ и из $(19),(20)$ легко вьвести, что гомоморфизм deg сопоставляет расслоению его первьй класс Чжэня.

Дивизором мероморфного дифференциала $\omega$ называется формальная сумма $(\omega)$ его нулей и полюсов, взятых, соответственно, с положительными и отрицательными знаками и с учетом кратности. Согласно теореме Абеля дивизоры мероморфных дифференциалов линейно эквивалентны. Этот класс эквивалентности назьвается каноническим классом поверхности и обозначается $C(\Gamma)$. Его описание дает следуюшее предложение. 
5.3.4. 1) $\operatorname{deg} C(\Gamma)=2 g-2$; 2) $A(C(\Gamma))=-2 K_{\Gamma}$.

Размерности $l(D)$ пространств $H^{0}(\Gamma ; \mathscr{O}([D]))=\mathscr{L}(D)$ (см. $\left.\S 4.1\right)$ описываются теоремой Римана-Роха.

\subsection{5. ТеоРема РИмана-Роха.}

$$
l(D)=\operatorname{deg} D-g+1+l(C(\Gamma)-D) .
$$

Для неспециальных эффективных дивизоров степени $g$ формула Римана-Роха принимает вид $l(D)=1$.

\section{4. Тройные секущие многообразий Якоби.}

5.4.1. ФОРМУЛА ТРОЙНОЙ СЕКУШЕЙ Фэя [36]. Для любых точек $p_{1}, p_{2}, p_{3} u p_{4}$ римановой поверхности $Г$ существуют постоянные $c_{1}(\bar{p}), c_{2}(\bar{p})$ и $c_{3}(\bar{p})$ такие, что

$$
\begin{array}{r}
c_{1} \cdot \theta\left(z+p_{1}-p_{3}\right) \cdot \theta\left(z+p_{2}-p_{4}\right)+c_{2} \cdot \theta\left(z+p_{1}-p_{4}\right) \cdot \theta\left(z+p_{2}-p_{3}\right) \\
+c_{3} \cdot \theta\left(z+p_{1}+p_{2}-p_{3}-p_{4}\right) \cdot \theta(z)=0 .
\end{array}
$$

Это әквивалентно тому, что точки многообразия Куммера

$$
\Phi\left(\frac{p_{1}+p_{2}-p_{3}-p_{4}}{2}\right), \Phi\left(\frac{p_{1}+p_{3}-p_{2}-p_{4}}{2}\right), \Phi\left(\frac{p_{1}+p_{4}-p_{2}-p_{3}}{2}\right)
$$

лежат на одной прямой $\mathbb{C P}^{1}$ (тройной секущей $)$ в $\mathbb{C P}^{2^{g}-1}$.

Здесь и ниже мы ради краткости обозначаем $A\left(p_{k}\right)$ через $p_{k}$.

Формула тройной секущей для четверки точек $\bar{p}$ общего положения следует из предложений 4.4.2 и 5.2.1 и теоремы Римана о тэта-дивизоре многообразия Якоби.

В случае общего положения мы можем считать, что

$$
\operatorname{dim} \Theta_{-\left(p_{1}-p_{3}\right)} \cap \Theta_{-\left(p_{1}-p_{4}\right)}=g-2
$$

(в действительности, это верно всегда [82]). Осталось показать, что

$$
\Theta_{-\left(p_{1}-p_{3}\right)} \cap \Theta_{-\left(p_{1}-p_{4}\right)} \subset \Theta_{-\left(p_{1}+p_{2}-p_{3}-p_{4}\right)} \cup \Theta .
$$

Пусть $\theta\left(u-p_{3}\right)=\theta\left(u-p_{4}\right)=0$ и $u=z+p_{1}$. Если $\theta(u-p)=0$ для всех $p \in \Gamma$, то $\theta(u)=0$. В противном случае $\theta(u-p)=0$ при $p=p_{3}, p_{4}, q_{1}, \ldots, q_{g-2}$, где $q_{j} \in \Gamma$ и $u=p_{1}+p_{2}+q_{1}+\cdots+q_{g-2}+K_{\Gamma}$. Но в этом случае $u^{\prime}=z+p_{1}+p_{2}-p_{3}-p_{4}=p_{2}+$ $q_{1}+\cdots+q_{g-2}+K_{\Gamma}$ и $\theta\left(u^{\prime}\right)=0$ согласно теореме Римана. Формула (90) доказана, и теперь достаточно применить предложение 4.4.2.

Для произвольных четверок $\bar{p}$ сушествование тройной секущей, проходящей через точки (89), получается из общего случая предельным переходом. Явные формулы для $c_{k}(\bar{p})$ могут быть получены из (88) (см. также [36]). 


\section{$\S$ 6. Тэта-функции многообразий Прима двулистных накрытий с двумя точками ветвления}

\section{1. Многообразия Прима разветвленных накрытий.}

Пусть $\Gamma_{0}$ - риманова поверхность рода $g$ и $q_{0}$ и $q_{\infty}$ - пара различных точек на ней. Обозначим через $\gamma_{0}$ и $\gamma_{\infty}$ контуры, ограничивающие маленькие круги с центрами в точках $q_{0}$ и $q_{\infty}$.

Множество двулистных накрытий $\Gamma \rightarrow \Gamma_{0}$, разветвленных в точках $q_{0}$ и $q_{\infty}$, находится во взаимно однозначном соответствии с множеством гомоморфизмов вида

$$
\rho: H_{1}\left(\Gamma \backslash\left\{q_{0}, q_{\infty}\right\} ; \mathbb{Z}\right) \rightarrow \mathbb{Z}_{2}
$$

таких, что $\rho\left(\left[\gamma_{0}\right]\right)=\rho\left(\left[\gamma_{\infty}\right]\right)=1$. Соответствие имеет следующий вид. Пусть $\gamma-$ контур на $\Gamma_{0}$. Если при его подъеме на накрытие он размькается, то положим $\rho([\gamma])=1$. В противном случае положим $\rho([\gamma])=0$. Из этого соответствия следует, что накрытие задается поверхностью $\Gamma_{0}$ и точками ветвления с точностью до $2^{2 g}$ возможностей.

Обозначим через $\mathscr{M}_{g, 2}$ пространство модулей римановых поверхностей с парой отмеченных точек, а через $\mathscr{D} \mathscr{R}_{g}$ пространство модулей двулистных накрытий поверхностей рода $g$ с двумя точками ветвления. Отображение $\Gamma \rightarrow\left(\Gamma_{0}, q_{0}, q_{\infty}\right)$ задает

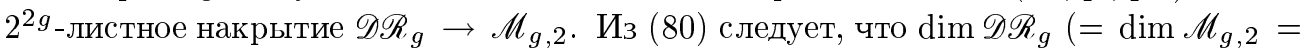
$\left.\operatorname{dim} \mathscr{M}_{g}+2\right)=3 g-1$ при $g \geqslant 2$.

Пусть $\pi: \Gamma \rightarrow \Gamma_{0}$ - накрытие, разветвленное в точках $q_{0}$ и $q_{\infty}$. Обозначим через $\sigma: \Gamma \rightarrow \Gamma$ инволюцию, переставляющую листы накрытия. Очевидно, что $\Gamma_{0}=\Gamma / \sigma$ и инволюция $\Gamma \stackrel{\sigma}{\longrightarrow} \Gamma$ однозначно задает накрытие.

На поверхности $\Gamma$ рода $2 g$ сушествует такой канонический базис 1-циклов $a_{1}, \ldots, a_{2 g}, b_{1}, \ldots, b_{2 g}$, что

$$
\sigma\left(a_{k}\right)+a_{g+k}=\sigma\left(b_{k}\right)+b_{g+k}=0, \quad 1 \leqslant k \leqslant g,
$$

и циклы $a_{1}, \ldots, a_{g}, b_{1}, \ldots, b_{g}$ проектируются в канонический базис циклов $\left\{\widetilde{a}_{k}, \widetilde{b}_{k}\right\}$ на $\Gamma_{0}$, где $\widetilde{a}_{k}-\pi_{*}\left(a_{k}\right)=\widetilde{b}_{k}-\pi_{*}\left(b_{k}\right)=0$.

Пусть $\omega_{1}, \ldots, \omega_{2 g}$ - базис голоморфных дифференциалов на $Г$ и $\widetilde{\omega}_{1}, \ldots \widetilde{\omega}_{g}-$ базис голоморфных дифференциалов на $\Gamma_{0}$, связанные с каноническими базисами циклов соотношениями (78). Из (91) следует, что

$$
\sigma^{*}\left(\omega_{k}\right)=-\omega_{g+k} \quad \text { и } \omega_{k}-\omega_{g+k}=\pi^{*}\left(\widetilde{\omega}_{k}\right) \text { при } 1 \leqslant k \leqslant g .
$$

Дифференщиал $\omega$ назьвается дифференииалом Прима, если $\sigma^{*}(\omega)=-\omega$. Формы $u_{1}=\omega_{1}+\omega_{g+1}, \ldots, u_{g}=\omega_{g}+\omega_{2 g}$ образуют базис голоморфных дифференциалов Прима. Рассмотрим матрищу их $b$-периодов:

$$
\Pi(\Gamma, \sigma)_{j k}\left(=\Pi_{j k}\right)=\int_{b_{k}} u_{j}, \quad 1 \leqslant j, k \leqslant g .
$$

Эта матрица симметрична и согласно (91), (92) связана с матрицами периодов поверхностей $Г$ и $\Gamma_{0}$ соотношением

$$
B(\Gamma)=\frac{1}{2}\left(\begin{array}{ll}
\Pi+B_{0} & \Pi-B_{0} \\
\Pi-B_{0} & \Pi+B_{0}
\end{array}\right),
$$


где

$$
B(\Gamma)_{j k}\left(=B_{j k}\right)=\int_{b_{k}} \omega_{j}, \quad B\left(\Gamma_{0}\right)_{j k}\left(=B_{0 j k}\right)=\int_{\pi_{*}\left(b_{k}\right)} \widetilde{\omega}_{j} .
$$

Главно поляризованное абелево многообразие

$$
\operatorname{Pr}(\Gamma, \sigma)=\mathbb{C}^{g} /\left\{\mathbb{Z}^{g}+\Pi \mathbb{Z}^{g}\right\}
$$

называется многообразием Прима накрытия $\Gamma \rightarrow \Gamma / \sigma$.

Многообразие Прима инвариантно определяется для любой инволюции $\sigma$ на $Г$ следуюшим образом. Пусть $A: \Gamma \rightarrow J(\Gamma)$ - отображение Абеля с начальной точкой $\bar{q}$ и $\sigma: \Gamma \rightarrow \Gamma$ - инволюция. Тогда $J(\Gamma)=A\left(S^{n} \Gamma\right)(n-$ род $Г)$ и инволюция $\sigma$ порождает инволюцию $\sigma: J(\Gamma) \rightarrow J(\Gamma)$ :

$$
\sigma\left(\int_{\bar{q}}^{q_{1}} \omega+\cdots+\int_{\bar{q}}^{q_{2 g}} \omega\right)=\int_{\sigma(\bar{q})}^{\sigma\left(q_{1}\right)} \omega+\cdots+\int_{\sigma(\bar{q})}^{\sigma\left(q_{2 g}\right)} \omega
$$

Многообразием Прима назьвается антиинвариантное подмногообразие

$$
\operatorname{Pr}(\Gamma, \sigma)=\{z \in J(\Gamma): \sigma(z)=-z\}
$$

В нашем случае из (92) следует, что

$$
\sigma\left(z_{1}, \ldots, z_{g}, z_{g+1}, \ldots, z_{2 g}\right)=\left(-z_{g+1}, \ldots,-z_{2 g},-z_{1}, \ldots,-z_{g}\right)
$$

и определено вложение

$$
\varphi: \operatorname{Pr}(\Gamma, \sigma) \rightarrow J(\Gamma), \quad \varphi\left(z_{1}, \ldots, z_{g}\right)=\left(z_{1}, \ldots, z_{g}, z_{1}, \ldots, z_{g}\right)
$$

Из (98) следует

6.1.1. Если $[\mu]$ - главная поляризация $J(\Gamma)$, то поляризачия $\varphi^{*}([\mu]) / 2$ является главной.

Таким образом, корректно определено отображение Прима

$$
\operatorname{Pr}: \mathscr{D} \mathscr{R}_{g} \rightarrow \mathscr{A}_{g}, \quad(\Gamma, \sigma) \stackrel{\operatorname{Pr}}{\longrightarrow}\left[\operatorname{Pr}(\Gamma, \sigma), \varphi^{*}([\mu]) / 2\right) .
$$

Аналоги предложения 6.1.1 выполняются еще для двух классов двулистных накрытий: неразветвленных и гиперэллиптических. В первом случае поляризация, индуцированная вложением, кратна главной, а во втором - многообразие Прима совпадает с многообразием Якоби гиперэллиптической римановой поверхности (двулистного разветвленного накрытия сферы).

\section{2. Тэта-функция многообразия Прима.}

Многообразие Якоби $J\left(\Gamma_{0}\right)$ изоморфно инвариантному подмногообразию инволюции $\sigma-J\left(\Gamma_{0}\right)=\{z \in J(\Gamma): \sigma(z)=z\}$, и вкладывается в $J(\Gamma)$ :

$$
\pi^{*}: J\left(\Gamma_{0}\right) \rightarrow J(\Gamma), \quad \pi^{*}\left(z_{1}, \ldots, z_{g}\right)=\left(z_{1}, \ldots, z_{g},-z_{1}, \ldots,-z_{g}\right) .
$$


Заданы и проекции

$$
\pi_{1}: J(\Gamma) \rightarrow \operatorname{Pr}(\Gamma, \sigma), \quad \pi_{2}: J(\Gamma) \rightarrow J\left(\Gamma_{0}\right),
$$

имеющие в координатах вид

$$
\pi_{1}(u, v)=u+v, \quad \pi_{2}(u, v)=u-v, \quad u, v \in \mathbb{C}^{g} .
$$

Композиция $\operatorname{Pr}(\Gamma, \sigma) \times J\left(\Gamma_{0}\right) \stackrel{\varphi+\pi^{*}}{\longrightarrow} J(\Gamma)$ вложений $\varphi$ и $\pi^{*}$ является изогенией степени $2^{2 g}$. Заметим, что

$$
2 e=\varphi \cdot \pi_{1}(e)+\pi^{*} \cdot \pi_{2}(e)
$$

Из (94) и (101) следует, что

6.2.1. 1) Для любых характеристик $a, b, c, d \in \mathbb{R}^{g}$

$$
\begin{aligned}
& \theta\left[\begin{array}{ll}
a & b \\
c & d
\end{array}\right](z, B) \\
& \quad=\sum_{2 e \in \mathbb{Z}^{g} / 2 \mathbb{Z}^{g}} \theta\left[\begin{array}{c}
(a+b) / 2+e \\
c+d
\end{array}\right]\left(\pi_{1}(z), 2 \Pi\right) \cdot \theta\left[\begin{array}{c}
(a-b) / 2+e \\
c-d
\end{array}\right]\left(\pi_{2}(z), 2 B_{0}\right) .
\end{aligned}
$$

2) Формула

$$
\frac{\theta(z, B)^{2}}{\theta\left(\pi_{1}(z), \Pi\right) \cdot \theta\left(\pi_{2}(z), B_{0}\right)}
$$

определяет мероморфную функцию на $J(\Gamma)$.

Отображением Абеля-Прима (с начальной точкой $q_{0}$ ) назьвается отображение

$$
A_{\operatorname{Pr}}: \Gamma \rightarrow \operatorname{Pr}(\Gamma, \sigma), \quad A_{\operatorname{Pr}}(q)_{j}=\int_{q_{0}}^{q} u_{j}
$$

Через $A_{0}$ и $A$ обозначим отображения Абеля для поверхностей $\Gamma_{0}$ и $\Gamma$ с начальными точками $\pi\left(q_{0}\right)$ и $q_{0}$, соответственно. Из их определений и (101) следует, что

$$
A_{\mathrm{Pr}}=\pi_{1} \cdot A, \quad A_{0} \cdot \pi=\pi_{2} \cdot A
$$

Также легко показать, что

$$
C(\Gamma)=\pi^{-1}\left(C\left(\Gamma_{0}\right)\right)+q_{0}+q_{\infty}
$$

Из предложений 5.2.1 (примененного к (103)), 6.2.1 и (106) следует 
6.2.2. Пусть $f(p)=\theta\left(A_{\operatorname{Pr}}(p)-e, \Pi\right)$. Для $e \in \operatorname{Pr}(\Gamma, \sigma)$ выполняется одна из двух возможнностей:

1) $f(p) \equiv 0$

2) если $D-$ - двизор нулей функиии $f(p)$, то

$$
\varphi(e)=A(D)-A\left(q_{0}\right)-A\left(q_{\infty}\right)+\pi^{*}\left(K_{\Gamma_{0}}\right),
$$

что влечет за собой

$$
D+\sigma(D)-q_{0}-q_{\infty} \approx C(\Gamma)
$$

При этом дивизор D определяется по (107) однозначно.

Предложение 6.2.2 описывает обращение (84) отображения Абеля, ограниченное на многообразие Прима $\varphi(\operatorname{Pr}(\Gamma, \sigma)) \subset J(\Gamma)$.

\section{3. Четверные секущие многообразий Прима.}

6.3.1. ФОРМУЛА ЧЕТВЕРНОЙ СЕКУЩЕЙ ФЭЯ [37]. Для любъх точек $p_{1}, p_{2}, p_{3}$, $p_{4} \in \Gamma$ существуют постоянные $c_{1}(\bar{p}), c_{2}(\bar{p}), c_{3}(\bar{p})$ и $c_{4}(\bar{p})$ такие, что

$$
\begin{aligned}
& c_{1} \cdot \theta\left(z-p_{1}-p_{2}\right) \cdot \theta\left(z-p_{3}-p_{4}\right)+c_{2} \cdot \theta\left(z-p_{1}-p_{3}\right) \cdot \theta\left(z-p_{2}-p_{4}\right) \\
& \quad+c_{3} \cdot \theta\left(z-p_{1}-p_{4}\right) \cdot \theta\left(z-p_{2}-p_{3}\right)+c_{4} \cdot \theta\left(z-p_{1}-p_{2}-p_{3}-p_{4}\right) \cdot \theta(z)=0
\end{aligned}
$$

Это әквивалентно тому, что точки $\Phi\left(\left(p_{1}+p_{2}-p_{3}-p_{4}\right) / 2\right), \Phi\left(\left(p_{1}+p_{3}-p_{2}-p_{4}\right) / 2\right)$, $\Phi\left(\left(p_{1}+p_{4}-p_{2}-p_{3}\right) / 2\right)$ u $\Phi\left(\left(p_{1}+p_{2}+p_{3}+p_{4}\right) / 2\right)$ многообразия Куммера $K(\operatorname{Pr}(\Gamma, \sigma), \Theta)$ лежат на одной комплексной проективной плоскости (четверной секущей) e $\mathbb{C P}^{2^{g}-1}$.

Здесь под $p_{i}$ понимается $A_{\mathrm{Pr}}\left(p_{i}\right)$, а $\theta(z)=\theta(z, \Pi(\Gamma, \sigma))$.

Как и в $§ 5.4$ достаточно показать, что для общей четверки точек

$$
X=\theta_{p_{1}+p_{2}} \cap \theta_{p_{1}+p_{3}} \cap \theta_{p_{1}+p_{4}} \subset \theta \cup \theta_{p_{1}+p_{2}+p_{3}+p_{4}}
$$

и $\operatorname{dim} X=g-3$. Мы ограничимся наброском доказательства (110), так как условие на размерность $X$ не является слишком ограничительным. Можно считать, что все точки $p_{1}, \ldots, p_{4}$ различны.

Пусть $u=z-p_{1}$. Из предложения 6.2 .2 и (110) следует, что 1) $\theta(u-p) \equiv 0$, или 2) $p_{2}, p_{3}, p_{4} \in D$, где $D$ - дивизор нулей функции $f(p)=\theta(z-p)$.

Если $\theta(u-p) \equiv 0$, то из (105) следует, что $z-p_{1}-\sigma\left(p_{1}\right)=z$ и $\theta(z)=\theta\left(u-\sigma\left(p_{1}\right)\right)=0$.

Пусть функция $\theta(u-p)$ не обрашается тождественно в нуль. Докажем, что тогда $\theta\left(z-\left(p_{1}+\cdots+p_{4}\right)\right)=0$. Положим $D_{1}=D_{0}-\left(p_{2}+p_{3}+p_{4}\right)$.

Дивизоры, удовлетворяюшие (108), образуют приводимое подмногообразие $M \subset S^{2 g} \Gamma$. На одной из компонент $M_{1} \subset M$ отображение Абеля $\widehat{A}: D \rightarrow$ $A\left(D-q_{0}-q_{\infty}\right)+K_{\Gamma_{0}}$ обратимо посредством (107). Дивизоры $\pi(D), D \in M_{1}$, образуют подмногообразие $M^{\prime} \subset S^{2 g} \Gamma_{0}$, и накрытие $M \rightarrow M^{\prime}$ разветвлено на подмногообразии $M_{0}^{\prime}$, образованном дивизорами нулей абелевых дифференциалов. Можно показать [105], что если взять замкнутьй путь в $M^{\prime} \backslash M_{0}^{\prime}$ и поднять его на $M_{1}$, то его концы будут различаться на дивизор вида $D-\sigma(D)$, где $\operatorname{deg} D$ четна. Отсюда 
заключаем, что дивизор $D_{2}=\sigma\left(p_{2}+p_{3}+p_{4}\right)+D_{1}$ не лежит в $M_{1}$ и поэтому в точке $\widehat{A}\left(D_{2}\right)$ отображение Абеля необратимо.

Но из предложения 6.2.2 и необратимости отображения Абеля в точке $\widehat{A}\left(D_{2}\right)$ следует, что $\theta\left(u^{\prime}-p\right) \equiv 0$, где $u^{\prime}=\widehat{A}\left(D_{2}\right)=u-p_{2}-p_{3}-p_{4}$. Отсюда вьводим, что $\theta\left(z-\left(p_{1}+\cdots+p_{4}\right)\right)=0$.

Включение (110) доказано.

Мы привели схему доказательства формулы четверной секущей, следуя [105]. Это доказательство схоже с доказательством формулы тройной секущей Фэя, изложенным выше, и демонстрирует геометрически их различие.

Впервые формула (109) была получена Фэем [37] с помошью теории соотношений Шоттки-Юнга.

\section{§ 7. Тэта-функции многообразий Прима неразветвленных накрытий}

\section{1. Многообразия Прима неразветвленных накрытий.}

Пусть $\Gamma_{0}$ - риманова поверхность рода $g$. Множество двулистных неразветвленных накрытий $\Gamma \rightarrow \Gamma_{0}$ находится во взаимно однозначном соответствии с множеством гомоморфизмов вида

$$
\rho: H_{1}(\Gamma ; \mathbb{Z}) \rightarrow \mathbb{Z}_{2}
$$

Соответствие имеет следующий вид. Пусть $\gamma-$ контур на $\Gamma_{0}$. Если при его подъеме на накрытие он размыкается, то положим $\rho([\gamma])=1$. В противном случае положим $\rho([\gamma])=0$. Из этого соответствия следует, что неразветвленное двулистное накрытие задается поверхностью $\Gamma_{0}$ с точностью до $2^{2 g+2}$ возможностей, причем для одного из них $(\rho \equiv 0)$ накрывающее пространство состоит из двух компонент.

Обозначим через $\mathscr{D} \mathscr{U}_{g}$ пространство модулей двулистных неразветвленных накрытий поверхностей рода $g$. Отображение $\Gamma \rightarrow \Gamma_{0}$ задает $2^{2 g}$-листное накрытие $\mathscr{D} \mathscr{U}_{g} \rightarrow \mathscr{M}_{g+1}$. Из (80) следует, что $\operatorname{dim} \mathscr{D} \mathscr{U}_{g}\left(=\operatorname{dim} \mathscr{M}_{g}\right)=3 g-3$ при $g \geqslant 2$.

Пусть $\pi: \Gamma \rightarrow \Gamma_{0}-$ неразветвленное двулистное накрытие. Обозначим через $\sigma: \Gamma \rightarrow \Gamma$ инволюцию, переставляюшую листы накрытия. Очевидно, что $\Gamma_{0}=\Gamma / \sigma$ и инволюция $\Gamma \stackrel{\sigma}{\longrightarrow} \Gamma$ однозначно задает накрытие.

На поверхности Г рода $2 g-1$ сушествует такой канонический базис 1 -циклов $a_{0}, b_{0}$, $a_{1}, \ldots, a_{2(g-1)}, b_{1}, \ldots, b_{2(g-1)}$, что

$$
\sigma\left(a_{0}\right)-a_{0}=\sigma\left(b_{0}\right)-b_{0}=\sigma\left(a_{k}\right)-a_{g-1+k}=\sigma\left(b_{k}\right)-b_{g-1+k}=0
$$

при $1 \leqslant k \leqslant g-1$, и циклы $a_{0}, b_{0}, a_{1}, \ldots, a_{g-1}, b_{1}, \ldots, b_{g-1}$ проектируются в канонический базис циклов $\left\{\widetilde{a}_{k}, \widetilde{b}_{k}\right\}$ на $\Gamma_{0}$, где $\widetilde{a}_{0}=\pi_{*}\left(a_{0}\right), \widetilde{b}_{0}=\pi_{*}\left(b_{0}\right)$ и $\widetilde{a}_{k}-\pi_{*}\left(a_{k}\right)=$ $\widetilde{b}_{k}-\pi_{*}\left(b_{k}\right)=0$ при $1 \leqslant k \leqslant g-1$.

Для такого базиса гомоморфизм $\rho$ примет простой вид:

$$
\rho\left(a_{j}\right)=0, \quad \rho\left(b_{j}\right)=0 \text { при } j \neq 0, \quad \rho\left(b_{0}\right)=1 .
$$

Пусть $\omega_{0}, \omega_{1}, \ldots, \omega_{2(g-1)}$ - базис голоморфных дифференциалов на $Г$ и $\widetilde{\omega}_{0}$, $\widetilde{\omega}_{1}, \ldots, \widetilde{\omega}_{g-1}-$ базис голоморфных дифференциалов на $\Gamma_{0}$, связанные с каноническими 
базисами циклов соотношениями (78). Из (112) следует, что $\sigma^{*}\left(\omega_{0}\right)=\omega_{0}, \sigma^{*}\left(\omega_{k}\right)=$ $\omega_{g-1+k}$ и $\omega_{k}+\omega_{g-1+k}=\pi^{*}\left(\widetilde{\omega}_{k}\right)$ при $1 \leqslant k \leqslant g-1$.

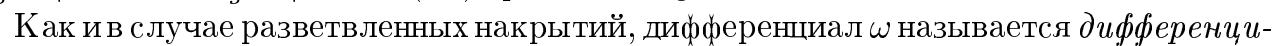
алом Прима, если $\sigma^{*}(\omega)=-\omega$. Формы $u_{k}=\omega_{k}+\omega_{g-1+k}, 1 \leqslant k \leqslant g-1$, образуют базис голоморфных дифференциалов Прима.

Многообразие Прима накрытия $\Gamma \rightarrow \Gamma_{0}$ определяется, как и в случае разветвленных накрытий, формулой (96) и имеет вид

$$
\operatorname{Pr}(\Gamma, \sigma)=\mathbb{C}^{g-1} /\left\{\mathbb{Z}^{g-1}+\Pi(\Gamma, \sigma) \mathbb{Z}^{g-1}\right\},
$$

где П $(\Gamma, \sigma)$ задается формулой (93). Однако связь между многообразиями Якоби и Прима примет иной вид

$$
B(\Gamma)=\left(\begin{array}{ccc}
T_{0} & T_{1} & T_{1} \\
T_{1}^{*} & \left(\Pi+T_{2}\right) / 2 & \left(\Pi-T_{2}\right) / 2 \\
T_{1}^{*} & \left(\Pi-T_{2}\right) / 2 & \left(\Pi+T_{2}\right) / 2
\end{array}\right),
$$

где

$$
B\left(\Gamma_{0}\right)=\left(\begin{array}{cc}
T_{0} / 2 & T_{1} \\
T_{1} & T_{2}
\end{array}\right), \quad T_{0}=\int_{b_{0}} \omega_{0}, \quad T_{1 k}=\int_{b_{k}} \omega_{0} .
$$

Инволюция $\sigma: J(\Gamma) \rightarrow J(\Gamma)$ принимает вид

$$
\sigma\left(z_{0}, z_{1}, \ldots, z_{g-1}, z_{g}, \ldots, z_{2(g-1)}\right)=\left(z_{0}, z_{g}, \ldots, z_{2(g-1)}, z_{1}, \ldots, z_{g-1}\right),
$$

и вложение многообразия Прима в $J(\Gamma)$ задается формулой

$$
\varphi: \operatorname{Pr}(\Gamma, \sigma) \rightarrow J(\Gamma), \quad \varphi\left(z_{1}, \ldots, z_{g}\right)=\left(0, z_{1}, \ldots, z_{g-1},-z_{1}, \ldots,-z_{g-1}\right) .
$$

Из (115) также следует

7.1.1. Если $[\mu]-$ главная поляризация $J(\Gamma)$, то форма $\varphi^{*}([\mu]) / 2$ задает главную поляризачию многообразия Прима $\operatorname{Pr}(\Gamma, \sigma)$.

Поэтому, как и в случае разветвленных накрытий, определено отображсение Примa

$$
\operatorname{Pr}: \mathscr{D} \mathscr{U}_{g} \rightarrow \mathscr{A}_{g-1}, \quad(\Gamma, \sigma) \stackrel{\operatorname{Pr}}{\longrightarrow}\left(\operatorname{Pr}(\Gamma, \sigma), \varphi^{*}([\mu]) / 2\right) .
$$

7.2. Тэта-функция многообразия Прима и формула четверных секущих для неразветвленных накрытий.

Многообразие Якоби $J\left(\Gamma_{0}\right)$ является $\sigma$-инвариантным подмногообразием $J(\Gamma)$ и вкладывается в него как

(117) $\pi^{8}: J\left(\Gamma_{0}\right) \rightarrow J(\Gamma)$,

$$
\pi^{*}\left(z_{0}, z_{1}, \ldots, z_{g-1}\right)=\left(2 z_{0}, z_{1}, \ldots, z_{g-1}, z_{1}, \ldots, z_{g-1}\right) .
$$

Определены проекции

$$
\pi_{1}: J(\Gamma) \rightarrow \operatorname{Pr}(\Gamma, \sigma), \quad \pi_{2}: J(\Gamma) \rightarrow J\left(\Gamma_{0}\right),
$$

задаюшиеся формулами

$$
\pi_{1}(u, v, w)=v-w, \quad \pi_{2}(u, v, w)=(u, v+w), \quad u \in \mathbb{C}, \quad v, w \in \mathbb{C}^{g} .
$$

Композиция $\operatorname{Pr}(\Gamma, \sigma) \times J\left(\Gamma_{0}\right) \stackrel{\varphi+\pi^{*}}{\longrightarrow} J(\Gamma)$ вложений $\varphi$ и $\pi^{*}$ является изогенией степени $2^{2 g-1}$ и

$$
2 e=\varphi \cdot \pi_{1}(e)+\pi^{*} \cdot \pi_{2}(e) .
$$

Сформулируем аналог предложения 6.2.1. 
7.2.1. Для любых $a_{0}, c_{0} \in \mathbb{R}, a, b, c, d \in \mathbb{R}^{g-1}$

$$
\begin{aligned}
& \theta\left[\begin{array}{lll}
a_{0} & a & b \\
c_{0} & c & d
\end{array}\right](z, B) \\
= & \sum_{2 e \in \mathbb{Z}^{g}-1 / 2 \mathbb{Z}^{g-1}} \theta\left[\begin{array}{cc}
a_{0} & (a+b) / 2+e \\
c_{0} & c+d
\end{array}\right]\left(\pi_{1}(z), 2 \Pi\right) \cdot \theta\left[\begin{array}{c}
(a-b) / 2+e \\
c-d
\end{array}\right]\left(\pi_{2}(z), 2 B_{0}\right) .
\end{aligned}
$$

Так как выше уже было показано, как доказьваются формулы секуших, мы изложим формулу Бовилля-Дебарра без доказательства. По этой же причине мы опустим аналог предложения 6.2.2 для неразветвленных накрытий.

Определим отображение Абеля-Прима

$$
A_{\mathrm{Pr}}: \Gamma \rightarrow \operatorname{Pr}(\Gamma, \sigma)
$$

формулой

$$
A_{\operatorname{Pr}}(p)_{j}=\frac{1}{2} \int_{\sigma(p)}^{p} u_{j}
$$

В [7] была получена

7.2.2. ФОРМУЛА ЧЕТВЕРНОЙ СЕКУЩЕЙ БОВИЛЛЯ-ДЕБАРРА. Для любъx moчек $p_{1}, p_{2}, p_{3}, p_{4} \in \Gamma$ точки $\Phi\left(\left(p_{1}+p_{2}-p_{3}-p_{4}\right) / 2\right), \Phi\left(\left(p_{1}+p_{3}-p_{2}-p_{4}\right) / 2\right)$, $\Phi\left(\left(p_{1}+p_{4}-p_{2}-p_{3}\right) / 2\right) u \Phi\left(\left(p_{1}+p_{2}+p_{3}+p_{4}\right) / 2\right)$ многообразия Куммера $K(\operatorname{Pr}(\Gamma, \sigma), \Theta)$ лежат на одной комплексной проективной плоскости в $\mathbb{C P}^{2^{g}-1}$.

\title{
ГЛАВА ІІІ \\ МНОГООБРАЗИЯ ЯКОБИ И СОЛИТОННЫЕ УРАВНЕНИЯ (ПРОБЛЕМА РИМАНА-ШОТТКИ, ГИПОТЕЗА НОВИКОВА, ТРОЙНЫЕ СЕКУЩИЕ)
}

\author{
$\S$ 8. Функции Бейкера-Ахиезера и кольца \\ коммутирующих дифференциальных операторов
}

8.1. Конечнозонные операторы и функции Бейкера-Ахиезера.

Функиией Бейкера-Ахиезера на поверхности Г (рода $g$ ), отвечающей точке $q \in \Gamma$, локальному параметру $k^{-1}$ в окрестности $q(k(q)=\infty)$, многочлену $Q(k)$ и дивизору $D=p_{1}+\cdots+p_{g}$, называется функция $\psi(p)$ такая, что

1) $\psi$ мероморфна на $\Gamma \backslash q$ и $(\psi) \geqslant-D$ (см. $\S 4.1)$;

$2)$ в окрестности точки $q$ функшия $\psi(p) \cdot \exp (-Q(k))$ аналитична. 
Обозначим через $\mathscr{S}=\left\{\Gamma, q, k^{-1}, Q(k), D\right\}$ набор “спектральных данных" и через $\Lambda(\mathscr{S})$ - пространство функций Бейкера-Ахиезера с такими данными. Из теоремы Римана-Роха следует, что

8.1.1. Если дивизор полюсов функиии $\psi$ неспециален, то для многочлена $Q$ общего положения ее дивизор нулей тоже неспециален и $\operatorname{dim} \Lambda(\mathscr{S})=1$.

Данное определение естественно обобщается на случай $n$-точечных или векторнозначных функций. Функции Бейкера-Ахиезера были введены Кричевером [61] и являются основным инструментом метода конечнозонного интегрирования солитонных уравнений.

В простейшем случае, когда Г-гиперэллиптическая поверхность $w^{2}=E^{2 g+1}+\cdots$, $q$ - бесконечно удаленная точка ветвления, $Q(k)=k x$ и $k=\sqrt{E}$, функция Бейкера-Ахиезера переходит в блоховскую собственную функцию оператора

$$
L=\frac{d^{2}}{d x^{2}}+u(x) .
$$

А именно, если оператор (122) периодичен $(u(x+T)=u(x))$, то функция $\psi(x, p)$ называется блоховской, если

$$
\begin{gathered}
L \psi=E(p) \cdot \psi ; \\
\psi(x+T, p)=\mu(p) \cdot \psi(x, p) .
\end{gathered}
$$

Здесь точка $p$ принадлежит двулистному накрытию $E$-плоскости, которое, таким образом, параметризует все собственные функции оператора (122), являюшиеся одновременно собственными для оператора сдвига $f(x) \rightarrow f(x+T)$.

Если накрытие $(E, \mu) \rightarrow E$ имеет конечное число точек ветвления, то можно показать, что их число будет нечетным, и точки $(E, \mu)$ образуют гиперэллиптическую поверхность

$$
w^{2}=E^{2 g+1}+\cdots
$$

на которой и определена блоховская функция $\psi(x, p)$. При $E \rightarrow \infty$ блоховская функция имеет асимптотику $\psi(x, p) \sim \exp (\sqrt{E} x) \cdot\left(1+O\left(E^{-1 / 2}\right)\right)($ см. [31]).

ОПРЕДЕЛЕНИЕ. Оператор (122) назьвается конечнозонным ( $g$-зонным $)$, если его блоховская функция определена на римановой поверхности конечного рода (рода $g$ ).

В общем случае оператор назьвается конечнозонным, если он обладает собственной функцией являющейся функцией Бейкера-Ахиезера (возможно, многоточечной и векторнозначной).

8.2. Конечнозонные решения уравнений Кортевега-де Фриза и Кадомцева-Петвиашвили.

Уравнение Кортевега-де Фриза (КдФ)

$$
u_{t}=\frac{1}{4}\left(6 u u_{x}+u_{x x x}\right)
$$


представляется в виде $L, A$-napbl - условия коммутации дифференциальных операторов:

$$
\left[L,-\frac{\partial}{\partial t}+A\right]=L_{t}+[L, A]=0
$$

где

$$
L=-\frac{\partial^{2}}{\partial x^{2}}+u(x, t), \quad A=\frac{\partial^{3}}{\partial x^{3}}-\frac{3}{2} u(x, t) \frac{\partial}{\partial x}-\frac{3}{4} \frac{\partial u(x, t)}{\partial x} .
$$

Сушествует бесконечное семейство дифференциальных операторов $A_{2 n+1}$ вида

$$
A_{2 n+1}=\frac{\partial^{2 n+1}}{\partial x^{2 n+1}}+\cdots
$$

такое, что

1) оно содержит оператор $A=A_{3}$ вида (125);

2 ) условия коммутации (124) для $A=A_{2 n+1}$ порождают на пространстве потенциалов $u(x)$ бесконечномерное семейство коммутируюших потоков, описываемых нелинейньми уравнениями типа КдФ (иерархия уравнений Кортевега-де Фриза) (см. [67]).

Сташионарные решения уравнений иерархии КдФ обладают замечательными свойствами:

8.2.1. (Новиков [90], Дубровин, Новиков [33]). Потенциал и(x) оператора (122) является конечнозонным тогда и только тогда, когда он является стачионарнылм решением какого-то уравнения иерархии КдФ:

$$
\left[L, A_{2 n+1}+c_{2 n-1} \cdot A_{2 n-1}+\cdots+c_{1} \cdot A_{1}\right]=0 .
$$

Oператор L имеет $g$ зон, если (126) выполняется при $n=2 g+1$ и не выполняется при меньших $n$. Конечнозонные операторы переходят под действием КдФ-потоков в конечнозонные.

В 1974 году в работах Новикова, Дубровина, Матвеева, Итса, Лакса класс конечнозонных решений КдФ был введен и изучен [90], [33], [55], [68] (в 1975 году некоторые результаты этих исследований были получены также в [76], иной подход к периодической задаче для КдФ был рассмотрен Марченко [74]). Подробньй обзор этих работ дан в [31]. Недавно Веселов и Шабат нашли очень интересную характеризацию конечнозонных операторов вида (122) с помошшю преобразований Дарбу [110]. Мы лишш кратко изложим процедуру построения конечнозонных решений Кд $\Phi$ (теоремы 8.2 .2 и 8.2 .3$)$.

Пусть $\Gamma=\left\{w^{2}=E^{2 g+1}+\cdots\right\}$ - гиперэллиптическая поверхность, $q=(E=\infty)$ - бесконечно удаленная точка ветвления, $k=\sqrt{E}$ и $Q(k)=k x$. Для неспециального дивизора $D=p_{1}+\cdots+p_{g}$ степени $g$ пространство $\Lambda(\mathscr{S})$ одномерно, где $\mathscr{S}=$ $\left\{\Gamma, q, k^{-1}, Q, D\right\}$.

Набор $\Sigma=\left\{\Gamma, q, k^{-1}, D\right\}$ называется набором “спектральных данных" оператора Шрёдингера (122). 
8.2.2. В случае общего положения $(\operatorname{dim} \Lambda(\mathscr{S})=1)$ существует единственный оператор $L$ вида (122) такой, что $L \psi=E \psi$, где $\psi \in \Lambda(\mathscr{S})$ u $\psi(x, k)=$ $\exp (k x)\left(1+O\left(k^{-1}\right)\right)$.

Докажем это утверждение. Пусть

$$
\psi(x, k)=\exp (k x) \cdot\left(1+\frac{\xi(x)}{k}+O\left(\frac{1}{k^{2}}\right)\right) .
$$

Тогда при $u=-2 \xi_{x}$ функция $\left(L-k^{2}\right) \psi$ принадлежит $\Lambda(\mathscr{S})$ и, так как $\operatorname{dim} \Lambda(\mathscr{S})=1$, то $\left(L-k^{2}\right) \psi=\lambda \cdot \psi$, где $\lambda$ - постоянная. Но $\exp (-k x) \cdot\left(L-k^{2}\right) \psi \rightarrow 0$ при $E \rightarrow \infty$. Отсюда следует, что $\lambda=0$ и $L \psi=E \psi$ при $u=-2 \xi_{x}$.

Фактически, мы получили решение обратной задачи - процедуру восстановления оператора $L$ по “спектральным данным" $\mathscr{S}$ :

$$
\mathscr{S} \rightarrow u(x)=-2 \xi_{x}
$$

Конечнозонные решения уравнения КдФ получаются теперь следуюшим образом. Преобразуем спектральные данные $\mathscr{S}$, заменив $Q(k)=k x$ на $Q_{1}(k)=k x+k^{3} t$, и построим по новым спектральным данным $\mathscr{S}^{\prime}$ функцию $\psi(x, t, k)$ такую, что

$$
\psi(x, k)=\exp \left(k x+k^{3} t\right) \cdot\left(1+\frac{\xi(x, t)}{k}+O\left(\frac{1}{k^{2}}\right)\right)
$$

Пусть

$$
u(x, t)=-2 \xi_{x}
$$

Функция $f=\left(-\partial_{t}+A\right) \psi$ принадлежит $\Lambda\left(\mathscr{S}^{\prime}\right)$ и тождественно равна нулю, так как $\exp \left(-k x-k^{3} t\right) \cdot\left(-\partial_{t}+A\right) \psi \rightarrow 0$ при $E \rightarrow \infty$. Значит, $\left[L,-\partial_{t}+A\right] \psi=0$, но оператор $\left[L,-\partial_{t}+A\right]$ является оператором умножения на скалярную функцию $f(x, t)=\left(u_{t}-\left(6 u u_{x}+u_{x x x}\right) / 4\right)$, а функция $\psi$ нетривиальна. Мы заключаем, что функция $f$ тождественно равна нулю и поэтому $u(x, t)=-2 \xi_{x}$ удовлетворяет уравнению КдФ (123). Итак, доказано, что

8.2.3. По набору спектральных данных общего положсения однозначно строятся конечнозонный оператор Шрёдингера (127)-(128) и конечнозонное решение уравнения Кортевега-де Фриза (129)-(130).

В [55] было показано, что это решение описьвается тэта-функциональной формулой

$$
u(x, t)=2 \partial_{x}^{2} \log \theta(U x+W t+Z, \Omega),
$$

где векторы $U, W$ постоянны, $\Omega=B(\Gamma)$ и $Z=-A(D)-K_{\Gamma}$. Вьвод этой формулы сейчас широко известен и изложен, например, в обзорах [31], [62], [27]. Функция $\psi$ является блоховской собственной функцией оператора $L$ и строится по нему явно, как и "спектральные данные" [31]. 
Уравнение Кадомцева-Петвиашвили (КП)

$$
\frac{3}{4} u_{y y}=\frac{\partial}{\partial x}\left(u_{t}-\frac{1}{4}\left(6 u u_{x}+u_{x x x}\right)\right)=0
$$

является условием коммутации

$$
\left[-\frac{\partial}{\partial_{y}}+L,-\frac{\partial}{\partial_{t}}+A\right]=0
$$

где

$$
L=\frac{\partial^{2}}{\partial x^{2}}+u(x, y, t), \quad A=\frac{\partial^{3}}{\partial x^{3}}+\frac{3}{2} u \frac{\partial}{\partial x}+w .
$$

Оно является двумеризацией уравнения $\mathrm{K} \Phi$, в которое переходит, если потенциал $u(x, y)$ не зависит от $y$.

8.2.4. (Кричевер [61], [62]). Пусть Г - произвольная риманова поверхность рода $g, q \in \Gamma, k^{-1}$ - локальныи параметр в окрестности $q, D=p_{1}+\cdots+p_{g}$ - неспециальный эффективный дивизор степени $g, Q(k)=k x+k^{2} y+k^{3} t u$ $\mathscr{S}=\left\{\Gamma, q, k^{-1}, Q(k), D\right\} . \quad B$ случае общего положсения $(\operatorname{dim} \Lambda(\mathscr{S})=1)$ функиия $\psi \in \Lambda(\mathscr{S})$ однозначно определяется условием $\exp (-Q(k)) \cdot \psi(x, y, t, k) \rightarrow 1$ при $k \rightarrow \infty$ и по ней однозначно восстанавливаются операторы $L$ и $A$ вида (122) такие, что

$$
\left(-\frac{\partial}{\partial y}+L\right) \psi=\left(-\frac{\partial}{\partial t}+A\right) \psi=0
$$

Формуль восстановления имеют вид

$$
u=-2 \frac{\partial \xi_{1}}{\partial x}, \quad w=3 \xi_{1} \frac{\partial \xi_{1}}{\partial x}+3 \frac{\partial^{3} \xi_{1}}{\partial x^{3}}-3 \frac{\partial \xi_{2}}{\partial x}
$$

əəe

$$
\psi(x, k)=\exp \left(k x+k^{2} y+k^{3} t\right) \cdot\left(1+\frac{\xi_{1}(x, t)}{k}+\frac{\xi_{2}(x, t)}{k^{2}}+O\left(\frac{1}{k^{3}}\right)\right)
$$

Функиия $u(x, y, t)$ является при этом решением уравнения КП и имеет вид

$$
u(x, y, t)=2 \partial_{x}^{2} \log \theta(U x+V y+W t+Z, \Omega),
$$

где векторы $U, V, W$ постоянны,$\Omega=B(\Gamma)$ и $Z=-A(D)-K_{\Gamma}$.

Доказательство этой теоремы аналогично изложенным выше доказательствам теорем 8.2.2 и 8.2.3. Существенно то, что для оператора $L$ вида (122) прямая задача построения блоховской функции не имеет эффективного решения (она эффективно решается еше для матричного оператора Дирака, что позволило в середине 70-х годов построить конечнозонные решения нелинейного уравнения Шрёдингера (Итс, Котляров) и уравнения sin-Gordon (Козел, Котляров), выражающиеся в тэта-функциях гиперэллиптических поверхностей (см. обзор [31]). Аксиоматизация аналитических 
свойств блоховских функций в определении функций Бейкера-Ахиезера позволяет строить широкий класс решений, минуя решение прямой задачи. Уравнение КП было первым, к которому эта схема (метод Кричевера) была применена. Более того, его конечнозонные решения строятся по любым римановьм поверхностям.

8.3. Коммутативные кольца обыкновенных линейных дифференциальных операторов.

Проблема описания коммутативных колец обькновенных линейных дифференщиальных операторов связана согласно теореме 8.2.1 с описанием класса конечнозонных операторов и была поставлена Новиковьм. Ее эффективное решение было получено Кричевером [61], [63] для коммутирующих операторов ранга 1, повторившим при этом ряд результатов Берчналла и Чонди [14].

Здесь мы под операторами будем понимать обькновенные линейные дифференциальные операторы.

Пара коммутируюших операторов $L_{1}$ и $L_{2}$ имеет ранг $l$, если для пары собственных значений $\left(\lambda_{1}, \lambda_{2}\right)$ общего положения размерность пространства функций $\varphi$ таких, что $L_{1} \varphi=\lambda_{1} \varphi, L_{2} \varphi=\lambda_{2} \varphi$, paвна $l$. В этом случае $l$ является наибольшим обшим делителем порядков операторов $L_{1}$ и $L_{2}$.

Под “спектральными данньми” $\Sigma$, как и вьше, будем понимать совокупность римановой поверхности $\Gamma$ рода $g$, точки $q \in \Gamma$, локального параметра $k^{-1}$ в окрестности $q$ и эффективного дивизора $D$ (общего положения) степени $g$. Пусть $\psi(x, p)-ф$ функция Бейкера-Ахиезера, отвечающая набору $\Sigma$ и однозначно нормированная условием $\exp (-k x) \cdot \psi(x, p) \rightarrow 1$ при $p \rightarrow q$.

8.3.1. Пусть $f$-мероморфная функиия на Г с единственным полюсом в точке q, $Q(k)$ - такой полином, что $f(k)=Q(k)+O(1)$. Тогда по функции $f$ однозначно строится оператор $L_{f}$ такой, что $L_{f} \psi(x, p)=f(p) \psi(x, p)$.

Доказательство теоремы 8.3.1 аналогично доказательствам теорем 8.2.2 и 8.2.4. В случае, когда $f$ имеет в $q$ полюс второго порядка, это в точности теорема 8.2.2. Оператор $L_{f}$ эффективно восстанавливается по формулам, аналогичньм (130), (134).

Обозначим через $M(q)=\bigcup_{d} \mathscr{L}(d q)$ алгебру мероморфных функций на $\Gamma$ с единственным полюсом в точке $q$.

8.3.2. Определенная выше прочедура $f \rightarrow L_{f}$ задает изоморфизм алгебрьи $M(q)$ с коммутативной алгеброй обыкновенных дифференииальных операторов. Пары операторов общего положения из этой алгебры имеют ранг 1.

Верно и обратное утверждение:

8.3.3. Любая максимальная коммутативная (ранга 1) алгебра обыкновенных дифференциальных операторов $R$ изоморфна алгебре $\left\{L_{f}\right\} \approx M(q)$ для каких-то спектральных данных $\Sigma$.

Алгебра $\left\{L_{f}\right\}$ строится явньм образом. Прежде всего, верно следующее предложение.

8.3.4. Если два обыкновенных дифференциальных оператора $L_{1}$ и $L_{2}$ коммутируют, то существует многочлен $Q(x, y)$ такой, что $Q\left(L_{1}, L_{2}\right)=0$.

Теперь с помошю формальных рядов строится расслоение общих собственных функций операторов $L_{1}$ и $L_{2}$ над римановой поверхностью $\Gamma=\{Q(x, y)=0\}$. 
Это расслоение одномерно и является функцией Бейкера-Ахиезера $\psi(x, p)$. Для полноты необходимо рассматривать также римановы поверхности с простейшими особенностями, но с несингулярньци точками $q$ (этот случай отдельно рассматривался в [14.III]). Поверхность $\Gamma \backslash q$ может быть инвариантно определена как спектр кольца $R: \Gamma \backslash q=\operatorname{Spec} R$.

Подробные доказательства всех этих результатов изложены в [61], [63]. Алгебраизации этой конструкции, в частности обобщаюшие ее на случай произвольного поля, даны в [26], [84]. Эффективного описания пары коммутирующих операторов ранга $l>1$ до сих пор не получено (см. обзоры [66], [91], [93]). Кричевер и Новиков нашли обший видпары ранга 2, отвечающей поверхности рода 1 ([65], [66], см. также [42], [92]), и с помошю их методов Мохов описал пары ранга 3 и рода 1 (см. [77], [78]). Случай матричных обыкновенных дифференциальных операторов был разобран Гриневичем [43].

\section{§. Гипотеза Новикова в проблеме Римана-Шоттки и тройные секущие многообразий Якоби}

\section{1. Эффективизация тэта-функциональных формул для конечнозон- ных решений.}

Входящие в тэта-функциональные формулы (131) и (136) для решений уравнений $\mathrm{K} д \Phi$ и $\mathrm{KП}$ векторы $U, V, W$ и постоянная $C$ определяются по спектральным данным с помошь трансцендентных уравнений. Новиков предложил эффективную процедуру построения этих формул, а именно, получить соотношения на матрицу Римана $\Omega=B(\Gamma) \in \mathscr{A}_{g}$ и векторы $U, V, W$ путем простой подстановки тэта-функциональных формул в уравнения $\mathrm{K} д \Phi$ и КП. При этом получится система алгебраических уравнений на векторы $U, V, W$, условия совместности которой дадут соотношения на матрищу Римана.

В случае малых размерностей $g=2,3$ никаких соотношений на матрицу Римана не будет. Это вытекает из следующей теоремы:

9.1.1. ТеОРема ТореллИ. Отображение $B: \mathscr{M} \rightarrow \mathscr{A}_{g}(81)$, сопоставляющее римановой поверхности ее многообразие Якоби, является вложением.

Геометрическое доказательство этой теоремы изложено, например, в [41], а солитонное - в [27]. Из нее следует, что $\operatorname{dim} A_{g}=\operatorname{dim} \mathscr{M}_{g}$ при $g=2,3$, и поэтому матрица $\Omega \in \mathscr{H}_{g}$ общего положения является матрищей периодов римановой поверхности. Укажем единственное ограничение.

9.1.2. Теорема МертенСа. Многообразие Якоби римановой поверхности неразложимо, т.е. не представляется в виде прямого произведения абелевых многообразий положительной размерности.

Условие неразложимости может быть эффективно описано на языке тэта-констант (значений тэта-функции и ее производных в нуле). 
9.1.3. [95]. Главно поляризованное абелево многообразие $\mathbb{C}^{g} /\left\{\mathbb{Z}^{g}+\Omega \mathbb{Z}^{g}\right\}$ неразложимо тогда и только тогда, когда ранг матрицы

$$
\left(\begin{array}{cccccc}
\widehat{\theta}_{11}\left[n_{1}, 0\right] & \ldots & \widehat{\theta}_{j k}\left[n_{1}, 0\right] & \ldots & \widehat{\theta}_{g g}\left[n_{1}, 0\right] & \widehat{\theta}\left[n_{1}, 0\right] \\
\ldots \ldots \ldots \ldots \ldots & \ldots \ldots \ldots \ldots \ldots \ldots \ldots \ldots \ldots \ldots \ldots \ldots \ldots \\
\widehat{\widehat{\theta}}_{11}\left[n_{r}, 0\right] & \ldots & \widehat{\theta}_{j k}\left[n_{r}, 0\right] & \ldots & \widehat{\theta}_{g g}\left[n_{r}, 0\right] & \widehat{\theta}\left[n_{r}, 0\right]
\end{array}\right)
$$

максимален, т.е. равен $g(g+1) / 2+1$. Здесь

$$
\widehat{\theta}[\alpha, \beta]=\theta[\alpha, \beta](0,2 \Omega), \quad \widehat{\theta}_{j k}[\alpha, \beta]=\frac{\partial^{2} \widehat{\theta}[\alpha, \beta](0,2 \Omega)}{\partial z^{j} \partial z^{k}}
$$

$u\left\{2 n_{j}\right\}=\mathbb{Z}^{g} / 2 \mathbb{Z}^{g}, r=2^{g}$.

Программа эффективного построения 2- и 3-зонных решений уравнений КдФ и КП была реализована Дубровиным. Основой ее явилось нахождение соотношений на векторы $U, V, W$.

9.1.4. (Дубровин [27]). Пусть абелево многообразие $\mathbb{C}^{g} /\left\{\mathbb{Z}^{g}+\Omega \mathbb{Z}^{g}\right\}$ неразложиммо.

1) Формула (131) задает при всех $Z$ решения уравнения Кортевега-де Фриза тогда и только тогда, когда при всех $n \in \frac{1}{2} \mathbb{Z}^{g} / 2 \mathbb{Z}^{g}$ выполняются соотношения

$$
\partial_{U}^{4} \widehat{\theta}[n, 0]-\partial_{U} \partial_{W} \widehat{\theta}[n, 0]+d \widehat{\theta}[n, 0]=0
$$

где $d$ - постоянная.

2) Формула (136) задает при всех $Z$ решения уравнения Кадомиева-Петвиашвили тогда и только тогда, когда при всех $n \in \frac{1}{2} \mathbb{Z}^{g} / 2 \mathbb{Z}^{g}$ выполняются соотношения

$$
\partial_{U}^{4} \widehat{\theta}[n, 0]-\partial_{U} \partial_{W} \widehat{\theta}[n, 0]+\partial_{V}^{2} \widehat{\theta}[n, 0]+d \widehat{\theta}[n, 0]=0
$$

где $d$ - постоянная.

Доказательство Дубровина подробно изложено в обзоре [27], и мы лишш отметим, что получение уравнений (139) и (140) состоит в подстановке тэта-формул в нелинейные уравнения и применении бинарной теоремы сложения Римана и теоремы 3.2.1. Как было впоследствии отмечено, эта подстановка приводит к уравнениям Хироты (196) на тэта-функщию, которые и сводятся к (139) и (140) после применения указанных теорем. Удобство формул (139) и (140) связано с возможностью их эффективного применения и к построению явных решений, и к проблемам алгебраической геометрии.

С помошњю соотношений (139) и (140) Дубровин получил прямую процедуру построения всех 2- и 3-зонных решений уравнения КП по произвольному неразложимому главно поляризованному абелеву многообразию размерности $g=2,3$. В случае уравнения КдФ такая процедура получается для $g=2$ (так как все римановы поверхности рода 2 гиперэллиптичны), а в роде 3 требуется дополнить процедуру эффективным 
выделением многообразий Якоби гиперэллиптических кривых. Это было также проделано в [27].

\section{2. Гипотеза Новикова в проблеме Римана-Шоттки.}

В случае $g \geqslant 4$ подмногообразие $J_{g}=B\left(\mathscr{M}_{g}\right)$ имеет положительную коразмерность в $\mathscr{A}_{g}$, равную $(g-2)(g-3) / 2$. Поэтому процедура построения решений уравнения КП с помошю формул эффективизации Дубровина (140) требует прежде на начальном шаге эффективного описания подмногообразия $B\left(\mathscr{M}_{g}\right)$. Это же являлось до начала 80-х годов одной из известнейших проблем алгебраической геометрии:

9.2.1. ПРОБЛЕМА РИМАНА-ШотТКИ. Найти уравнения, описьвающие замыкание множсества многообразий Якоби - $\bar{J}_{g}=\overline{B\left(\mathscr{M}_{g}\right)}-$ в пространстве модулей главно поляризованных абелевых многообразий $\mathscr{A}_{g}$.

До гипотезы Новикова нетривиальное соотношение было известно лишш при $g=4$ : оно было найдено в конще 19-го века Шоттки, показавшим, что оно выделяет в $\mathscr{A}_{4}$ подмногообразие (возможно, приводимое) коразмерности 1 , содержащее $\bar{J}_{g}(\S 10.1)$. В 1981 году Игуса доказал, что данное подмногообразие неприводимо и, тем самым, соотношение Шоттки решает проблему Римана-Шоттки для $g=4$ [54].

В конще 70-х годов Новиков выдвинул следующую гипотезу, инициированную конструкцией Кричевера конечнозонных решений уравнения КП по произвольной римановой поверхности (см. $\S 8.2)$.

9.2.2. ГИПОТЕЗА НовИКОВА. Неразложимое главно поляризованное абелево многообразие $M=\mathbb{C}^{g} /\left\{\mathbb{Z}^{g}+\Omega \mathbb{Z}^{g}\right\}$ является многообразием Якоби римановой поверхности тогда и только тогда, когда существуют такие векторы $U \in \mathbb{C}^{g} \backslash\{0\}$, $V, W \in \mathbb{C}^{g}$, что функция

$$
\mathscr{U}_{Z}(x, y, t)=2 \frac{\partial^{2}}{\partial x^{2}} \log \theta(U x+V y+W t+Z)
$$

является при любом $Z \in \mathbb{C}^{g}$ решением уравнения Кадомщева-Петвиашвили.

Согласно этой гипотезе соотношения, решающие проблему Римана-Шоттки, являются в точности соотношениями, выделяющими такие матрицы $\Omega \in \mathscr{H}_{g}$, при которых уравнения (140) разрешимы. Довольно скоро было получено частичное доказательство этой гипотезы:

9.2.3. (Дубровин [28]). Условие разрешимости соотношений (140) выделяет в $\mathscr{A}_{g}$ подмногообразие (возможно, приводимое) размерности $3 g-3=\operatorname{dim} \mathscr{M}_{g}$.

Теорема Дубровина дает, как и теорема Шоттки при $g=4$, локальное решение проблемы Римана-Шоттки, но при любых $g \geqslant 4$. Глобальное решение было бы получено, если бы удалось доказать, что данное подмногообразие неприводимо.

Полное доказательство гипотезы Новикова, а с ним и решение проблемы Римана-Шоттки было получено Шиотой [98]. Ему предшествовали важные работы Арбарелло-де Кончини [2] и Муласе [79]. Мы изложим все эти результаты в $§ 10$. 
9.3. Формула тройной секущей Фэя и конечнозонные решения солитонных уравнений.

Прежде, чем перейти к обсуждению проблемы Римана-Шоттки, мы изложим метод построения конечнозонных решений уравнения КП с помощью формулы тройной секушей Фэя. Этот метод, предложенный Мамфордом в начале 80-х годов [82], указывает на то, как уравнение КП возникает как вырождение чисто алгебро-геометрического тождества Фэя. Мы изложим его, следуя работе [3], в которой он был применен к проблеме Римана-Шоттки.

Обозначим через $\vec{\theta}(z)$ вектор $\left(\theta\left[n_{1}, 0\right](z, 2 \Omega), \ldots, \theta\left[n_{r}, 0\right](z, 2 \Omega)\right)$. Через $u \wedge v$ обозначим произведение векторов $u, v \in \mathbb{C}^{2^{g}}$ в алгебре Грассмана, порожденной $\mathbb{C}^{2^{g}}$.

Пусть $\Omega=B(\Gamma)$ и $p_{1}, p_{2}, p_{3}, p_{4} \in \Gamma$, где $\Gamma$ - произвольная риманова поверхность рода $g$. Согласно формуле тройной секущей $\Phi э я ~$

$$
\vec{\theta}\left(p_{1}+p_{2}-p_{3}-p_{4}\right) \wedge \vec{\theta}\left(p_{1}+p_{3}-p_{2}-p_{4}\right) \wedge \vec{\theta}\left(p_{1}+p_{4}-p_{2}-p_{3}\right)=0,
$$

где через $p_{k}$ мы обозначаем $A\left(p_{k}\right)$ и $A$ - отображение Абеля (83).

Можем считать, что точка $p_{1}$ является начальной точкой в определении отображения Абеля и $A\left(p_{1}\right)=0$. Выберем в окрестности точки $p_{1}$ локальный параметр $k^{-1}$ такой, что $k\left(p_{1}\right)=\infty$, и разложим отображение Абеля в окрестности $p_{1}$ в ряд по степеням $k^{-1}$ :

$$
A: k^{-1} \rightarrow \sum_{m=1}^{\infty} \frac{U_{m}}{k^{m}}
$$

$U_{m} \in \mathbb{C}^{g}$. Через $D_{m}$ обозначим производную в направлении $U_{m}$ :

$$
D_{m}=U_{m}^{1} \frac{\partial}{\partial z^{1}}+\cdots+U_{m}^{g} \frac{\partial}{\partial z^{g}}
$$

Введем формальный оператор $T(k)$ :

$$
T\left(k^{-1}\right)=\exp \left(\sum_{m=1}^{\infty} \frac{D_{m}}{k^{m}}\right)=\sum_{j \geqslant 0} \frac{S_{j}\left(D_{1}, \ldots\right)}{k^{j}} .
$$

Рассмотрим предел соотношения (141) при $p_{4} \rightarrow p_{1}: k^{-1}\left(p_{4}\right)=\alpha \rightarrow 0$. Разложим левую часть (141) в ряд по степеням $\alpha$. Первьй нетривиальньй член будет при $\alpha$ :

$$
\vec{\theta}\left(p_{2}-p_{3}\right) \wedge D_{1} \vec{\theta}\left(p_{2}-p_{3}\right) \wedge \vec{\theta}\left(p_{2}+p_{3}\right)=0
$$

Рассмотрим теперь предел (143) при $p_{3} \rightarrow p_{1}: k^{-1}\left(p_{3}\right)=\beta \rightarrow 0$, и опять разложим левую часть (143) в ряд по степеням $\beta$. Первьй нетривиальньй член будет при $\beta^{2}$ :

$$
\vec{\theta}\left(p_{2}\right) \wedge D_{1} \vec{\theta}\left(p_{2}\right) \wedge\left(D_{1}^{2}+D_{2}\right) \vec{\theta}\left(p_{2}+p_{3}\right)=0 .
$$

Как отметил Мамфорд [82], тождество (144) было указано в книге Фэя [36] (следствие 2.13).

Рассмотрим окончательньй предел $p_{2} \rightarrow p_{1}: k^{-1}\left(p_{2}\right)=\varepsilon \rightarrow 0$ и разложим левую часть (144) в ряд по степеням $\varepsilon$. Любой член этого ряда будет задавать линейное соотношение на значения в нуле функций $\theta[n, 0](z, 2 \Omega)$ и их частных производных, причем 
коэффициенты этого линейного соотношения не будут зависеть от характеристики $n$. Символически эти уравнения можно записать в виде

$$
\left.T(\varepsilon) \circ\left(\vec{\theta}(z) \wedge D_{1} \vec{\theta}(z) \wedge\left(D_{1}^{2}+D_{2}\right) \vec{\theta}(z)\right)\right|_{z=0} \equiv 0 .
$$

Заметим, что мы использовали, не оговаривая, то, что векторы $\vec{\theta}(u)$ и $\vec{\theta}(-u)$ равны и производные нечетного порядка функщий $\theta[n, 0](z, 2 \Omega)$ в точке $z=0$ равны нулю.

Как было отмечено в [2], уравнения (145) сводятся к уравнениям эффективизации Дубровина типа (139)-(140). После перенормировки $D_{j}$ (в случае необходимости) система

$$
\left.\Delta_{j}\left(\vec{\theta}(z) \wedge D_{1} \vec{\theta}(z) \wedge\left(D_{1}^{2}+D_{2}\right) \vec{\theta}(z)\right)\right|_{z=0}=0, \quad j \leqslant n,
$$

где $\Delta_{j}=S_{j}\left(D_{1}, \ldots\right)$, примет вид

$$
\left.\left(\Delta_{j} \cdot D_{1}-\Delta_{j-1} \cdot\left(D_{1}^{2}+D_{2}\right)+\sum_{m=3}^{j} d_{m+1} \Delta_{j-m}\right) \vec{\theta}(z)\right|_{z=0}=0, \quad j \leqslant n
$$

где $d_{1}, d_{2}, \ldots$ - постоянные. Припишем каждому дифференцированию $D_{j}$ его степень следуюшим образом:

$$
\operatorname{deg} D_{j}=j
$$

что вполне согласуется с определением $D_{j}$ как производной по $j$-му члену разложения отображения Абеля. Тогда старший член (147) примет вид

$$
\left.\left(\Delta_{j} \cdot D_{1}-\Delta_{j-1} \cdot\left(D_{1}^{2}+D_{2}\right)\right) \vec{\theta}(z)\right|_{z=0}=0
$$

Именно старшие члены являются основньми, так как инвариантны при заменах локального параметра $k^{-1}$.

9.3.1. [82], [3]. Уравнения (145) и (147), полученные из тождества тройной секущей Фәя вырождением $p_{2}, p_{3}, p_{4} \rightarrow p_{1}$, являются уравнениями әффективизаиии для тэта-функииональных решений иерархии нелинейных уравнений (иерархии КП). Конечнозонные решения этих уравнений задаются формулой

$$
\mathscr{U}_{Z}\left(x, t_{1}, t_{2}, \ldots\right)=2 \frac{\partial^{2}}{\partial x^{2}} \log \theta\left(U_{1} x+U_{2} t_{1}+U_{3} t_{2}+\cdots+Z, B(\Gamma)\right) .
$$

Простейшее из этих уравнений совпадает с уравнением КП, решения которого даются формулой

$$
2 \frac{\partial^{2}}{\partial x^{2}} \log \theta\left(U_{1} x+\sqrt{3} U_{2} y+3 U_{3} t+Z, B(\Gamma)\right) .
$$


Если поверхность Г гиперэллиптична, точка $p_{1}$ является неподвижной точкой гиперэллиптической инволючии $\sigma$ и локальный параметр $k^{-1}$ обращается инволющией $(\sigma(k)=-k)$, то $U_{2}=0$ и иерархия (151) является иерархией КдФ.

Вырождениями формулы тройной секущей для гиперэллиптических поверхностей получается и иерархия уравнений sin-Gordon. Конечнозонные решения уравнения sin-Gordon

$$
\frac{\partial^{2} u}{\partial x \partial y}=\sin u
$$

строятся по гиперэллиптическим римановым поверхностям Г. Пусть $p_{1}$ и $p_{2}-$ различные точки ветвления гиперэллиптического накрытия $\Gamma \rightarrow \mathbb{C P}^{1}$. Уравнение sin-Gordon и его конечнозонные решения получаются из формулы тройной секущей в пределе $p_{3} \rightarrow p_{1}, p_{4} \rightarrow p_{2}$.

Введем в окрестностях точек $p_{1}$ и $p_{2}$ локальные параметры $k_{1}^{-1}$ и $k_{2}^{-1}$, обращаемые гиперэллиптической инволюцией $\sigma\left(k_{j}\right)=-k_{j}$. Через $U_{j}$ обозначим производную отображения Абеля в точке $p_{j}$ по параметру $k_{j}^{-1}$, а через $D_{j}$ - дифференцирование по направлению $U_{j}$.

Рассмотрим теперь предел (141) при $p_{4} \rightarrow p_{2}: k_{2}^{-1}\left(p_{3}\right)=\alpha \rightarrow 0$, и разложим левую часть (141) в ряд по $\alpha$ : член при $\alpha$ имеет вид

$$
\vec{\theta}\left(p_{1}-p_{3}\right) \wedge D_{2} \vec{\theta}\left(p_{1}-p_{3}\right) \wedge \vec{\theta}\left(p_{1}+p_{3}-2 p_{2}\right)=0 .
$$

Теперь положим $k_{1}^{-1}\left(p_{3}\right)=\beta \rightarrow 0$ и, разложив левую часть (153) в ряд по $\beta$, получим ряд, начинающийся с линейного члена с коэффициентом

$$
\vec{\theta}(0) \wedge D_{1} D_{2} \vec{\theta}(0) \wedge \vec{\theta}(2 \delta)=0,
$$

где $\delta=p_{1}-p_{2}$. Из (154) следует, что сушествуют постоянные $c_{1}, c_{2}$ и $c_{3}$ такие, что

$$
\begin{aligned}
& c_{1} \cdot \theta[n, 0](0,2 \Omega) \cdot \theta[n, 0](2 z, 2 \Omega) \\
& \quad+c_{2} \cdot \theta[n, 0](2 \delta, 2 \Omega) \cdot \theta[n, 0](2 z, 2 \Omega) \\
& \quad+c_{3} \cdot D_{1} D_{2} \theta[n, 0](0,2 \Omega) \cdot \theta[n, 0](2 z, 2 \Omega)=0
\end{aligned}
$$

где $\Omega=B(\Gamma)$. Анализируя тождество Фэя подробнее, можно показать, что все постоянные $c_{j}$ не равны нулю. Применим теперь к (155) теорему сложения Римана (52) и получим

$$
c_{1}+c_{2} \frac{\theta(z-\delta) \cdot \theta(z+\delta)}{\theta(z)^{2}}+\frac{c_{3}}{2} D_{1} D_{2} \log \theta(z)=0
$$

где $\theta(z)=\theta(z, B(\Gamma))$.

Так как точки $p_{1}$ и $p_{2}$ - точки ветвления гиперэллиптического накрытия, то легко показать, что $\delta$ - полупериод: $\delta=\left(m_{1}+B(\Gamma) m_{2}\right) / 2, m_{1}, m_{2} \in \mathbb{Z}^{g}$, где $g-$ род Г. Возьмем теперь тождества (156) для $z=u$ и $z=u+\delta$ и вьгтем одно из другого. После перенормировки постоянных получим:

$$
D_{1} D_{2} \log \frac{\theta(u+\delta)^{2}}{\theta(u)^{2}}=C^{\prime} \cdot\left(\frac{\theta(u+2 \delta) \theta(u)}{\theta(u+\delta)^{2}}-\frac{\theta(u+\delta) \theta(u-\delta)}{\theta(u)^{2}}\right) .
$$

С учетом того, что $\delta$ - полупериод, мы приходим к следующему выводу. 
9.3.2. [82]. Функиия

$$
\mathscr{U}_{Z}=2 \sqrt{-1} \log \frac{\theta\left(U_{1} x+U_{2} y+\delta+Z, B(\Gamma)\right)}{\theta\left(U_{1} x+U_{2} y+Z, B(\Gamma)\right)}-2 \pi\left(m, U_{1} x+U_{2} y+Z+\frac{\delta}{2}\right)
$$

при любом $Z \in \mathbb{Z}^{g}$ удовлетворяет уравнению $\sin -G o r d o n$

$$
\frac{\partial^{2} \mathscr{U}}{\partial x \partial y}=C \cdot \sin \mathscr{U}
$$

где $C$ - некоторая ненулевая постоянная.

Решение (158) было найдено в [60]. Тождество (156), которое “содержит” в себе и уравнение sin-Gordon, и его конечнозонные решения, было получено Фэем в [36] (предложение 2.10). Уравнения, эффективизируюшие тэта-функциональные формулы для решений уравнения sin-Gordon, были получены в [9], [32].

Заметим, мы привели заведомо не все возможные уравнения, "содержашиеся" в формуле тройной секущей, так как, во-первых, рассмотрели не все возможные вырождения и, во-вторых, рассматривая вырождения тождеств, ограничивались, как при получении (143) или (144), только первыми нетривиальными членами рядов, тождественно равных нулю.

\section{$\S$ 10. Проблема Римана-Шоттки}

\section{1. Соотношения Шоттки-Юнга.}

Пусть $\Gamma_{0}$ - риманова поверхность рода $g$. Рассмотрим двулистное неразветвленное накрытие $\Gamma \rightarrow \Gamma_{0}=\Gamma / \sigma$. Выберем для него (как в $\S 7$ ) такой канонический базис циклов, что гомоморфизм (111) примет вид

$$
\rho\left(a_{j}\right)=0, \quad \rho\left(b_{j}\right)=0 \text { при } j \neq 0, \quad \rho\left(b_{0}\right)=1 .
$$

Для краткости обозначим $B(\Gamma)$ через $\tau$ и $\operatorname{Pr}(\Gamma, \sigma)$ через $\pi$.

Мы ограничимся случаем, когда все характеристики имеют порядок 2: если $\varepsilon=\left(\varepsilon^{1}, \ldots, \varepsilon^{2 n}\right)-$ характеристика, то $2 \varepsilon^{j} \in\{0,1\}$. В этом случае характеристика $\left(\varepsilon_{1}, \varepsilon_{2}\right)\left(\varepsilon_{j} \in \mathbb{R}^{n}\right)$ тэта-функции от $n$ переменных назьвается четной, если число $4 \sum_{j} \varepsilon_{1}^{j} \varepsilon_{2}^{j}$ четно, и нечетной в противном случае.

10.1.1. СООТНОШЕНИЯ ПРОПОРЦИОНАЛЬНОСТИ ШоТТКИ-ЮНГА. Для четных характеристик $\left(\varepsilon_{1}, \varepsilon_{2}\right)$ отношение

$$
\frac{\theta\left[\begin{array}{l}
\varepsilon_{1} \\
\varepsilon_{2}
\end{array}\right](0, \pi)^{2}}{\theta\left[\begin{array}{ll}
0 & \varepsilon_{1} \\
0 & \varepsilon_{2}
\end{array}\right](0, \tau) \cdot \theta\left[\begin{array}{ll}
0 & \varepsilon_{1} \\
1 & \varepsilon_{2}
\end{array}\right](0, \tau)}=k^{2}(\pi, \tau)
$$

постоянно (не зависит от характеристики).

Эта теорема была доказана Шоттки для случая $g=4$ в 1888 году, но сами соотношения были в общем виде вьписаны в 1909 году в совместной работе Шоттки и 
Юнга [96], см. также обзор [34], где они были сформулированы в качестве гипотезы. Доказательство этих соотношений было получено Фаркашем и Раухом [35].

Схема их применения к проблеме Римана-Шоттки следующая:

1) над любой римановой поверхностью $\Gamma_{0}$ построим двулистное неразветвленное накрытие, как указано вьше;

2) возьмем однородное тождество, которому удовлетворяют все тэта-функции, например, тернарную теорему сложения Римана (54), и применим его к тэта-функциям Прима $\theta(z, \pi)$;

$3)$ подставим в данное тождество вместо значений $\theta[\alpha, \beta](z, \pi)$ в нуле пропорциональные им значения тэта-функций (с характеристиками) поверхности $\Gamma$, используя (159).

Окончательное тождество будет выполняться не для всех тэта-функций, т.е. будет не аналитическим, а геометрическим по своей природе.

Первое нетривиальное тождество и было найдено Шоттки для $g=4$. Современное изложение его вывода приведено в [18]. Игуса доказал, что оно в точности выделяет $\bar{J}_{4}$ [54]. Позднее было доказано, что соотношений Шоттки-Юнга хватает для локального решения проблемы Римана-Шоттки.

10.1.2. (Ван Геемен [39]). Для любого $g \geqslant 4$ соотношения Шоттки-Юнга вьделяют в $\mathscr{A}_{g}$ подмногообразие размерности $3 g-3$, одна из неприводимых компонент которого совпадает $с \bar{J}_{g}$.

Как показал Донаги, это подмногообразие приводимо и содержит дополнительные компоненты, отличные от $\bar{J}_{g}$ уже при $g=5$ [23].

10.2. Геометрия тэта-дивизора многообразия Якоби и проблема Римана-Шоттки.

Все подходы к проблеме Римана-Шоттки, разрабатьвавшиеся до гипотезы Новикова, опирались на описание тэта-дивизора многообразия Якоби, данное Риманом. В двух из них это выявилось особо ярко.

Первьй из них основан на изучении Sing $\Theta$, множества особых точек тэта-дивизора, описание которого для случая многообразий Якоби тоже было дано Риманом. Согласно доказанной им теореме об особенностях (см. [41]) это множество достаточно массивно. Подробное его изучение привело к следуюшему результату.

10.2.1. (Андреотти, Майер [1]). Условие $\operatorname{dim} \operatorname{Sing} \Theta \geqslant g-4$ выделяет в $\mathscr{A}_{g}$ подмногообразие размерности $3 g-3$ (возможно, приводимое), одна из компонент которого совпадает $c \bar{J}_{g}$.

Как и в случае соотношений Шоттки-Юнга, это подмногообразие приводимо: уже при $g=4$ оно содержит две компоненты (Бовилль, [5]). Отметим, что условие $\operatorname{dim} \operatorname{Sing} \Theta=g-3$ выделяет в $\bar{J}_{g}$ в точности многообразия Якобигиперэллиптических поверхностей.

Другой подход, восходяший к работам Ли и Виртингера, использует представление тэта-дивизора как сдвинутой суммы $g-1$ экземпляров вложенной поверхности $Г$ :

$$
\Theta=C+\cdots+C+\kappa
$$


где $C=A(\Gamma)$ - поверхность, вложенная в $J(\Gamma)$, и $\kappa=K_{\Gamma}$. Подмногообразие абелева многообразия, представленное в виде (160), называется гиперповерхностью транслячии. Изучение таких подмногообразий было начато Ли. Из одной теоремы Ли-Виртингера следует:

10.2.2. Главно поляризованное абелево многообразие тогда и только тогда является многообразием Якоби негиперәллиптической поверхности, когда в окрестности некоторой своей неособой точки тэта-дивизор имеет два различных представления (160).

Вопрос о том, как эффективно описать условие теоремы 10.2.2 уравнениями, обсуждался Пуанкаре и Чеботаревым, однако своего окончательного решения пока не получил (см. обзор [72]).

10.3. Тройные секущие многообразий Якоби.

Еще один подход к проблеме Римана-Шоттки был предложен Ганнингом [45], [46] и основан на тождестве тройной секущей Фэя.

Прежде, чем изложить теорему Ганнинга, укажем критерий Мацусаки-Хойта [75], [52], который, грубо говоря, состоит в следующем. Пусть $M$-абелево многообразие комплексной размерности $g, X \subset M$-эффективньй дивизор и $C-2$-цикл в $M$. Через $D$ обозначим оператор двойственности Пуанкаре: $D: H_{2 g-k}(M ; \mathbb{Z}) \rightarrow H^{k}(M ; \mathbb{Z})$. Пусть $\omega=D(X)$. Тогда следующие утверждения эквивалентны:

а) $\left(\omega^{g},[M]\right)=g !$ и $\omega^{g-1}=(g-1) ! D(C)$;

б) абелево многообразие $M$ является прямьм произведением многообразий Якоби римановых поверхностей $\Gamma_{1}, \ldots, \Gamma_{n}$ и

$$
\begin{aligned}
& X=\bigcup_{j}\left(J\left(\Gamma_{1}\right) \times \cdots \times J\left(\Gamma_{j-1}\right) \times \Theta_{j} \times J\left(\Gamma_{j+1}\right) \times \cdots \times J\left(\Gamma_{n}\right)\right), \\
& C=\bigcup_{j}\left(0 \times \cdots \times 0 \times \Gamma_{j} \times 0 \times \cdots \times 0\right),
\end{aligned}
$$

где $\Theta_{j}$ - тэта-дивизор $J\left(\Gamma_{j}\right)$.

Критерий Мацусаки-Хойта дает и описание замькания $\bar{J}_{g}$ как состоящего из прямых произведений многообразий Якоби.

Рассуждения Ганнинга основаны на следующей жесткости тройных секущих многообразий Якоби. Пусть $M=\mathbb{C}^{g} /\left\{\mathbb{Z}^{g}+\Omega \mathbb{Z}^{g}\right\}$ - главно поляризованное абелево многообразие. Для каждой тройки точек $\alpha, \beta, \gamma \in \mathbb{C}^{g}$ обозначим через $V_{2 \alpha, 2 \beta, 2 \gamma}$ множество таких $t \in \mathbb{C}^{g}$, что векторы $\Phi(t / 2+\alpha), \Phi(t / 2+\beta)$ и $\Phi(t / 2+\gamma)$ (где отображение Куммера определяется по формулам (62), (70)) компланарны.

10.3.1. [36], [44]. Если $M$-многообразие Якоби римановой поверхности Г, то

$$
V_{2 \alpha, 2 \beta, 2 \gamma}=\left\{t \in \mathbb{C}^{g}: t+\alpha+\beta+\gamma \in \widetilde{\Gamma}\right\}
$$

где $\widetilde{\Gamma}-$ прообраз $\Gamma=A(\Gamma) \subset M$ при проекции $\mathbb{C}^{g} \rightarrow M$.

Тождество тройной секущей (88)-(89) дает нам, в точности, $\alpha=p_{2}, \beta=p_{3}, \gamma=p_{4}$ и $t=\left(p_{1}-p_{2}-p_{3}-p_{4}\right)$. Отсюда следует и красивая процедура: если нам известны 
координаты трех точек общего положения, лежаших на $\Gamma \subset J(\Gamma)$, то по ним мы можем полностью восстановить риманову поверхность $\Gamma \subset J(\Gamma)$.

Если же для какого-то неразложимого главно поляризованного абелева многообразия $M$ и точек $\alpha, \beta, \gamma \in M$ множество $V_{2 \alpha, 2 \beta, 2 \gamma}$ не пусто, то Ганнинг доказал, что при вьполнении дополнительных ограничений $V_{2 \alpha, 2 \beta, 2 \gamma}$ содержит в качестве компоненты риманову поверхность $\Gamma$, для которой $M=J(\Gamma)$ (это было показано с помошњю критерия Мацусаки-Хойта):

10.3.2. ТЕОРемА ГАННИНГА [45]. Неразложимое главно поляризованное абелево многообразие $M$ размерности $g$ является многообразием Якоби римановой поверхности тогда и только тогда, когда существуют такие точки $\alpha, \beta, \gamma \in$ $\mathbb{C}^{g}$, чтоо

1) они представляют различные точки $M=\mathbb{C}^{g} /\left\{\mathbb{Z}^{g}+\Omega \mathbb{Z}^{g}\right\}$;

2) не существует комплексных умножений $F: M \rightarrow M$ таких, что $F(\alpha-\gamma)=F(\beta-\gamma)=0$

3) $\operatorname{dim}_{(-\alpha-\beta)} V_{2 \alpha, 2 \beta, 2 \gamma}>0$.

Комплексным умножением назьвается эндоморфизм $M$, порожденный линейным отображением $t \rightarrow c t+d, t, d \in \mathbb{C}^{g}, c \in \mathbb{C}$. Вьполнимость условия 3) влечет за собой существование поверхности $\Gamma$ как компоненты $V_{2 \alpha, 2 \beta, 2 \gamma}$, проходящей через точку $(-\alpha-\beta)$.

Критерий Ганнинга был впоследствии усилен Велтерсом [112]-[114] и Дебарром [22]. Велтерс вывел также его инфинитезимальньй вариант. Не желая углубляться в теорию схем, сформулируем его в огрубленной форме: если $M-$ неразложимое главно поляризованное абелево многообразие и для какой-то точки $\alpha$ “предельное подмногообразие $\lim _{\beta, \gamma \rightarrow \alpha} V_{2 \alpha, 2 \beta, 2 \gamma}$ " имеет положительную размерность в какой-то точке, то $M$ - многообразие Якоби римановой поверхности. На язык уравнений этот критерий был перенесен Арбарелло и де Кончини [2]:

10.3.3. Неразложимое главно поляризованное абелево многообразие $M$ (размерности $g$ ) является многообразием Якоби римановой поверхности, если и только если существуют такие векторь $U_{1} \neq 0, U_{2}, \ldots \in \mathbb{C}^{g}$, что для нихи тэта-функиии $M$ выполняются уравнения (145). Более того, достаточно ограничиться уравнениями

$$
\left.T(\varepsilon) \circ\left(\vec{\theta}(z) \wedge D_{1} \vec{\theta}(z) \wedge\left(D_{1}^{2}+D_{2}\right) \vec{\theta}(z)\right)\right|_{z=0} \equiv 0 \bmod \varepsilon^{N+1}
$$

при $N=\left[(3 / 2)^{g} \cdot g !\right]$.

Отсюда и из теоремы 9.3.1 следует

10.3.4. (Арбарелло-де Кончини [2], Муласе [79]). Неразложимое главно поляризованное абелево многообразие $M$ (размерности $g$ ) является многообразием Якоби римановой поверхности тогда и только тогда, когда функиии (150) при всех $Z \in \mathbb{C}^{g}$ являются решениями первых $N(g)(<\infty)$ уравнений иерархии Кадомцева-Петвиашвили.

Мы не указьваем формулу для $N(g)$, так как верхняя оценка Арбарелло-де Кончини уже указана в теореме 10.3.3, а Муласе [79], давший “солитонное" доказательство 
теоремы 10.3.4, вообще лишь ограничился указанием на существование такой оценки. Если бы в теореме 10.3.4 удалось бы верхнюю оценку для $N(g)$ снизить до $N(g)=2$, то тем самьм было бы получено доказательство гипотезы Новикова. Это и было сделано Шиотой (см. §10.5).

10.4. О солитонном доказательстве теоремы 10.3.4.

Высшие уравнения КП - это те уравнения, которым удовлетворяет функция $u\left(x, t_{1}, \ldots\right)=-2 \xi_{x}(128)$ для спектральных данных уравнения КП с многочленом $Q(k)=k x+k^{2} t_{1}+k^{3} t_{2}+\cdots$ (здесь мы трактуем $y$ как временную переменную $t_{1}$ ).

Дадим строгое определение этой иерархии. Пусть $R$ - кольцо гладких функций от $x, t_{1}, \ldots$ Введем алгебру $E$ формальных псевдодифференциальных операторов вида

$$
A=\sum_{-\infty}^{N<\infty} p_{n}\left(\frac{d}{d x}\right)^{n}, \quad p_{n} \in R,
$$

умножение в которой определено правилом Лейбнища

$$
\left(\frac{d}{d x}\right)^{k} \cdot f=\sum_{j \geqslant 0}\left(\begin{array}{l}
k \\
j
\end{array}\right) \frac{d^{k} f}{d x^{k}} \cdot\left(\frac{d}{d x}\right)^{k-j}, \quad k \in \mathbb{Z}, \quad f \in R .
$$

Порядок $\operatorname{ord} A$ оператора (163) равен $d$, если $p_{d} \neq 0$ и $p_{j}=0$ при $j \geqslant d$. Через $E^{-}$ обозначим подалгебру, образованную операторами порядка $<0$. Для каждого оператора $A$ однозначно определено разложение $A=A^{+}+A^{-}$в сумму дифференщиального оператора $A^{+}$и оператора $A^{-} \in E^{-}$.

Пусть $H$ - аффинное подпространство $E$, образованное операторами вида $L=d / d x+\psi$, где $\psi \in E^{-}$. Иерархия Кадомиева-Петвиашвили - это следующая система эволюционных уравнений по параметрам $t_{1}, \ldots$ на $H$ :

$$
\frac{\partial}{\partial t_{n}} L=\left[B_{n}, L\right], \quad B_{n}=\left(L^{n}\right)^{+} .
$$

Из определения этих потоков после несложных рассуждений следует

10.4.1. Иерархия КП әквивалентна следующей системе уравнений Захарова-Шабата:

$$
\frac{\partial B_{m}}{\partial t_{n}}-\frac{\partial B_{n}}{\partial t_{m}}=\left[B_{n}, B_{m}\right] .
$$

Векторные поля на $H$, задаваемые высшими уравнениями КП, коммутируют.

Действие КП-потоков (168) на потенщиалы $u\left(x, t_{1}, \ldots\right)$ операторов $L$ линеаризуется согласно формуле (150) для их конечнозонных решений (заметим, что здесь мы интерпретируем пространственную координату $y$ в (150) как первое из времен $\left.-t_{1}\right)$.

Пусть

$$
L=\frac{d}{d x}+\frac{\mathscr{U}}{2}\left(\frac{d}{d x}\right)^{-1}+\sum_{k=2} u_{k}\left(\frac{d}{d x}\right)^{-k} .
$$


Если $L$ удовлетворяет (165), то все функции $u_{k}$ выражаются как полиномы от функции $\mathscr{U}$ и ее производных. Поэтому естественно назьвать решением иерархии КП функцию $\mathscr{U}$. Именно это и подразумевается в формулировке гипотезы Новикова. Отсюда следует, что,

$$
\frac{\partial L}{\partial \tau}=0 \text { при } \frac{\partial \mathscr{U}}{\partial \tau}=0
$$

где $\tau=c_{1} t_{1}+\cdots+c_{n} t_{n}, c_{j} \in \mathbb{C}$.

Теперь становится ясным, как вывести теорему 10.3.4 из теории Берчналла-ЧондиКричевера (§ 8.3). Пусть $\theta(z, \Omega)$ - тэта-функция от $g$ переменных и функция

$$
\mathscr{U}_{Z}\left(x, t_{1}, t_{2}, \ldots\right)=2 \frac{\partial^{2}}{\partial x^{2}} \log \theta\left(U x+V_{1} t_{1}+V_{2} t_{2}+\cdots+Z, \Omega\right)
$$

является при всех $Z \in \mathbb{C}^{g}$ решением первых $N(g)=2 g+1$ уравнений иерархии КП. Тогда существуют такие линейные комбинации, что

$$
c_{1} V_{1}+\cdots+c_{2 m} V_{2 m}+V_{2 m+1}=d_{1} V_{1}+\cdots+d_{2 n-1} V_{2 n-1}+V_{2 n}=0
$$

и $2 m+1$ взаимно просто с $2 n$. Возьмем линейные операторы

$$
B^{\prime}=c_{1} B_{1}+\cdots+c_{2 m} B_{2 m}+B_{2 m+1}, \quad B^{\prime \prime}=d_{1} B_{1}+\cdots+d_{2 n-1} B_{2 n-1}+B_{2 n}
$$

Пусть $\tau^{\prime}=c_{1} t_{1}+\cdots+c_{2 m} t_{2 m}+t_{2 m+1}, \tau^{\prime \prime}=d_{1} t_{1}+\cdots+d_{2 n-1} t_{2 n-1}+t_{2 n}$. Согласно (165) и (168)

$$
\frac{\partial L}{\partial \tau^{\prime}}-\frac{\partial L}{\partial \tau^{\prime \prime}}=\left[B^{\prime}, B^{\prime \prime}\right]=0
$$

Из (172) заключаем, что так как операторы $B^{\prime}$ и $B^{\prime \prime}$ коммутируют и имеют взаимно простые порядки, то они включаются в максимальную коммутативную алгебру дифференщиальных операторов $\mathscr{A}$ ранга 1 . Из теоремы 8.3 .3 следует, что эта алгебра получается конструкцией Берчналла-Чонди-Кричевера по римановой поверхности Г и дополнительным спектральным данньм. Осталось доказать, что $\Omega=B(\Gamma)$.

Из того, что алгебра $\mathscr{A}$ максимальна, вьводится, что векторы $U, V_{1}, V_{2}, \ldots$ порождают касательное пространство к $J(Г)$ (это строго объяснено в [98]). Теперь из того, что формальное решение (169) снимается с конечномерного тора $\mathbb{C}^{g} /\left\{\mathbb{Z}^{g}+\Omega \mathbb{Z}^{g}\right\}$, мы вьводим, что поверхность Г гладкая. Так как согласно конструкции Кричевера по "спектральным данным" алгебры $\mathscr{A}$ в то же время можно построить конечнозонные решения вида (136), (150) уравнений иерархии КП, то легко теперь заключить, что эти решения в точности даются формулами (169).

Мы опустим строгие доказательства (см. [79], [98]), так как главное - откуда при "солитонном" доказательстве появляется риманова поверхность - мы уже рассказали. 
10.5. Теорема Шиоты (доказательство гипотезы Новикова).

Из теоремы 10.3.4 Шиота вывел доказательство гипотезы Новикова, показав, что если функция

$$
\mathscr{U}_{Z}\left(x, t_{1}, t_{2}\right)=2 \frac{\partial^{2}}{\partial x^{2}} \log \theta\left(U x+V_{1} t_{1}+V_{2} t_{2}+Z, \Omega\right)
$$

удовлетворяет первым двум уравнениям иерархии КП (это эквивалентно тому, что после замены $t_{1} \rightarrow y, t_{2} \rightarrow t$ она будет удовлетворять уравнению КП (132)), то можно последовательно построить векторы $V_{3}, V_{4}, \ldots$ такие, что функция (169) будет удовлетворять высшим уравнениям КП [98]. Доказательство Шиоты аналитично. В [3] Арбарелло и де Кончини упростили его доказательство.

10.5.1. Теорема Шиоты. Пусть $M=\mathbb{C}^{g} / \Lambda$ - неразложимое главно поляризованное абелево многообразие и $\theta$ - его тәта-функиия. Тогда соотношения (140) на $U, V, W \in \mathbb{C}^{g}, U \neq 0$, разрешимь тогда и только тогда, когда $M$ является многообразием Якоби римановой поверхности.

10.6. О новых подходах к описанию $\bar{J}_{g}$. Гипотеза Велтерса о тройной секущей.

Хотя Шиота и получил полное решение проблемы Римана-Шоттки, она не перестала привлекать внимание. Вьше мы указали на пять различных подходов (соотношения Шоттки-Юнга, геометрия Sing $\Theta$, поверхности транслящии, тройные секушие, гипотеза Новикова), из которых лишь последний привел к успеху. Теорема 10.3.3 (Велтерса-Арбарелло-де Кончини), указывая на глубокую связь тройных секуших и солитонных уравнений, является веским аргументом в пользу следуюшей гипотезы:

10.6.1. ГИПОТЕЗА ВЕЛТЕРСА. Неразложимое главно поляризованное абелево многообразие является многообразием Якоби тогда и только тогда, когда оно (или, точнее, его многообразие Куммера) имеет тройную секущую.

Несмотря на то, что утверждение гипотезы является исключительно сильным, она уже получила серьезные подтверждения.

10.6.2. (Бовилль, Дебарр [6]). Если неразложимое главно поляризованное абелево многообразие имеет тройную секущую, то Sing $\Theta \geqslant g-4$.

С учетом теоремы 10.2.1 (Андреотти-Майера) это означает, что условие гипотезы Велтерса решает проблему Римана-Шоттки локально - выделяет в $\mathscr{A}_{g}$ подмногообразие, одна из компонент которого совпадает с $\bar{J}_{g}$.

Опираясь на теорему 10.6.2, Дебарр показал, что

10.6.3. (Дебарр [21]). Если неразложимое главно поляризованное абелево многообразие является многообразием Прима неразветвленного двулистного накрытия и имеет тройную секущую, то оно является многообразием Якоби римановой поверхности.

Такие многообразия Прима образуют $3 g$-мерное семейство, и отображение Прима

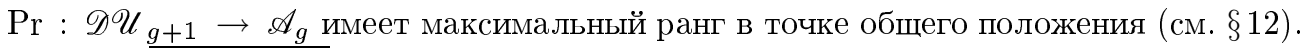
Поэтому $\operatorname{Pr}\left(\mathscr{D} \mathscr{U}_{g+1}\right)=\mathscr{A}_{g}$ при $g=4,5$. Отсюда следует 
10.6.4. (Дебарр [21]). Гипотеза Велтерса верна для 4- и 5-мерных абелевых многообразий.

Отметим еще два подхода к описанию $\bar{J}_{g}$ :

1) Красивое геометрическое свойство $\bar{J}_{g}$ было найдено недавно Бузером и Сарнаком: при больших $g$ подмногообразие $\bar{J}_{g}$ лежит в малой окрестности границы пространства $\mathscr{A}_{g}[15]$. Методы, использованные при его доказательстве, далеки от рассматриваемых в этом обзоре.

2) Также недавно Бухштабер и Кричевер предложили программу солитонного описания $\bar{J}_{g}$, основанную на функциональных уравнениях, выделяющих функции Бейкера-Ахиезера, и теоремах сложения для тэта-функций. Она находится в стадии разработки и пока не завершена [12], [13].

Иные геометрические подходы к решению проблемы Римана-Шоттки, связанные с предыдушими и основанные на изучении многообразий Куммера, рассматривались в $[86],[40]$.

\section{МНОГООБРАЗИЯ ПРИМА И НЕЛИНЕЙНЫЕ УРАВНЕНИЯ}

\section{$\S$ 11. Солитонные уравнения и тэта-функции Прима}

11.1. Конечнозонные двумерные операторы Шрёдингера и уравнение Веселова-Новикова.

Прямому обобшению схемы конечнозонного интегрирования Кд $\Phi$ на $2+1$-уравнения препятствует ряд обстоятельств.

Уравнение КдФ интегрируется в тэта-функциях римановой поверхности $Г$, параметризующей блоховские функции одномерного оператора Шрёдингера. Для $n$-мерного оператора Шрёдингера $\Delta+u$ глобальная блоховская функция определена на $n$-мерном комплексном многообразии $M^{n}$ следуюшего вида. Предположим для простоты, что потенциал $u\left(x^{1}, \ldots, x^{n}\right)-\mathbb{Z}^{n}$-периодичен. Пусть $\left(\mu_{1}, \ldots, \mu_{n}\right) \in \mathbb{C}^{n}$ и $E \in \mathbb{C}$. Условие существования блоховской функции $\psi\left(x^{1}, \ldots, x^{n}\right)$ с мультипликаторами $\mu$ $\left(u\left(x^{1}, \ldots, x^{j}+1, \ldots, x^{n}\right)=\mu_{j} \cdot u\left(x^{1}, \ldots, x^{j}, \ldots, x^{n}\right)\right)$ и собственным значением $E$ записьвается как условие непустоты ядра оператора

$$
L_{\nu, E}=\Delta+\sum_{j} \nu_{j} \partial_{x^{j}}+\left(u+\sum_{j} \nu_{j}^{2}-E\right)
$$

(где $\left.\mu_{j}=\exp \eta_{j}\right)$ на пространстве $W_{2}^{2}\left(\mathbb{R}^{n} / \mathbb{Z}^{n}\right)$. Это эквивалентно непустоте ядра оператора

$$
L_{\nu, E} \circ \Delta^{-1}: L_{2}\left(\mathbb{R}^{n} / \mathbb{Z}^{n}\right) \rightarrow L_{2}\left(\mathbb{R}^{n} / \mathbb{Z}^{n}\right)
$$

Оператор (174) имеет вид $1+P(\nu, E)$, где $P$ - компактньй оператор, полиномиально зависяший от $\nu, E$. Согласно теореме Келдыша необратимость такого оператора 
эквивалентна обрашению в нуль его детерминанта (определенного для таких операторов) и детерминант будет целой функцией от $\nu$ и $E$. Множество нулей детерминанта и будет многообразием $M^{n}$.

Естественным аналогом конечнозонности будет условие, что многообразие $M^{n}$ является проективно-алгебраическим. Однако схема интегрирования, при которой нелинейное уравнение задает деформацию блоховской собственной функции, здесь не проходит. Блоховская функщия на римановой поверхности определяет линейное расслоение, по которому восстанавливается оператор и которое деформируется под действием уравнения (см. $\S 8.3)$. В случае римановых поверхностей эта схема проходит, так как их групшы Пикара не дискретны (см. теорему 5.3.3), а при $n=2-$ нет, так как группы Пикара двумерных алгебраических многообразий дискретны.

Обойти эту трудность можно, зафиксировав, например, уровень энергии $E=\mathrm{const}$ при $n=2$. При этом $M^{2}$ заменяется одномерной комплексной поверхностью: “спектром" оператора $L$ на уровне әнергии $E$. Это проходит для уравнения КП с $L=-\partial_{y}+\partial_{x}^{2}+u(x, y)$ (см. [64]). То, что в оператор $L$ дифференцирование по $y$ входит линейно не случайно. Манаков показал, что если два дифференщиальных оператора от нескольких переменных образуют нетривиальную $L, A$-пару, то в каждњй из них дифференщирования по всем переменным кроме одной входят линейно [73].

Манаков предложил обобшение $L, A$-пар - $L, A, B$-тройки:

$$
\frac{\partial L}{\partial t}=[L, A]+B L
$$

Такие деформации сохраняют “спектр” $L$ на уровне энергии $E=0$.

Естественным кандидатом на роль деформируемого оператора $L$ является двумерньй оператор Шрёдингера

$$
\frac{\partial^{2}}{\partial x^{2}}+\frac{\partial^{2}}{\partial y^{2}}+V(x, y)
$$

Развитие теории двумерных операторов Шрёдингера, конечнозонных на одном уровне энергии, было начато в 1976 году Дубровиным, Кричевером и Новиковым [29] с решения для них обратной задачи.

11.1.1. 1) (Дубровин, Кричевер, Новиков [29]). Пусть Г-риманова поверхность рода $g, q_{1}$ u $q_{2}$ - пара различных точек $\Gamma, k_{1}^{-1}$ u $k_{2}^{-1}$ - локальнье параметры в их окрестностях, $D$ - эффективный неспечиальный дивизор степени g. Двухточечная функиия Бейкера-Ахиезера $\psi(z, \bar{z}, p)$, отвечающая этим спектральным данным и с асимптотиками

$$
\begin{aligned}
& \psi(z, \bar{z}, p)=\exp \left(k_{1} z\right) \cdot\left(1+\frac{\xi_{1}^{+}}{k_{1}}+O\left(\frac{1}{k_{1}^{2}}\right)\right), \quad p \rightarrow q_{1} \\
& \psi(z, \bar{z}, p)=c(z, \bar{z}) \cdot \exp \left(k_{2} \bar{z}\right) \cdot\left(1+O\left(\frac{1}{k_{1}}\right)\right), \quad p \rightarrow q_{2}
\end{aligned}
$$

единственна и является собственной функцией оператора

$$
L=\partial \bar{\partial}+A(z, \bar{z}) \cdot \bar{\partial}+V(z, \bar{z}),
$$


отвечающей нулевому собственному значению:

$$
L \psi=0 .
$$

Коэффициенты оператора восстанавливаются по формулам

$$
A(z, \bar{z})=-\partial \log c(z, \bar{z}), \quad V(z, \bar{z})=-\bar{\partial} \xi_{1}^{+} .
$$

Потенциал $V(z, \bar{z})$ имеет вид

$$
V(z, \bar{z})=2 \partial \bar{\partial} \log \theta\left(\widetilde{U}^{+} z+\widetilde{U}^{-} \bar{z}+\widetilde{Z}(D), B(\Gamma)\right) .
$$

2) (Чередник [16]). Если при этом существует антиголоморфная инволюиия $\tau: \Gamma \rightarrow \Gamma$ такая, что $\tau\left(q_{1}\right)=q_{2}, \tau\left(k_{1}\right)=-\bar{k}_{2} u \tau(D)+D-q_{1}-q_{2} \approx C(\Gamma)$, то оператор L самосопряжен.

3) (Веселов, Новиков [108]). Если жсе существует голоморфная инволючия $\sigma: \Gamma \rightarrow \Gamma$ такая, что $\sigma\left(q_{j}\right)=q_{j}, \sigma\left(k_{j}\right)=-k_{j} u$

$$
\sigma(D)+D-q_{1}-q_{2} \approx C(\Gamma)
$$

то оператор $L$ потенциален: $A \equiv 0$, и потенциал выражсается через тэта-функцию Прима разветвленного накрытия $\Gamma \rightarrow \Gamma / \sigma$ следующим образом:

$$
V(z, \bar{z})=2 \partial \bar{\partial} \log \theta\left(U^{+} z+U^{-} \bar{z}+Z(D), \operatorname{Pr}(\Gamma, \sigma)\right) .
$$

Указанные условия вещественности и потенциальности совместимы при $\sigma \tau=\tau \sigma \quad$ u $\tau(D)=D$. Уравнение (182) разрешимо, только если накрытие $\Gamma \rightarrow \Gamma / \sigma$ имеет лишь две точки ветвления (для гладких римановьх поверхностей).

Здесь $\partial=\partial / \partial z, \bar{\partial}=\partial / \partial \bar{z}, z=x+\sqrt{-1} y \in \mathbb{C}$. Вывод тэта-функциональных формул (181), (183) аналогичен выводу формулы (131).

Для гладких периодических операторов условия потенциальности, найденные Веселовым и Новиковым, являются и необходимыми (различные доказательства даны в [64] и в статье автора (в журнале “Функциональньй анализ и его приложения", 1990 , т. 24 , вып. 1 , с. 86-87)).

Уравнения, описьвающие “изоспектральные" деформации потенциальных операторов, образуют иерархию уравнений Веселова-Новикова.

11.1.2. (Веселов, Новиков [109]). Функиия Бейкера-Ахиезера с теми же спектральнымм данными, что и в 11.1.1.3), и асимптотиками

$$
\begin{aligned}
& \psi\left(z, \bar{z}, t^{\prime}, t^{\prime \prime}, p\right)=\exp \left(k_{1} z+\sum k_{1}^{2 m+1} t_{m}^{\prime}\right) \cdot\left(1+\frac{\xi_{1}^{+}}{k_{1}}+O\left(\frac{1}{k_{1}^{2}}\right)\right), p \rightarrow q_{1} \\
& \psi\left(z, \bar{z}, t^{\prime}, t^{\prime \prime}, p\right)=c(z, \bar{z}) \cdot \exp \left(k_{2} z+\sum k_{2}^{2 n+1} t_{n}^{\prime \prime}\right) \cdot\left(1+O\left(\frac{1}{k_{1}}\right)\right), p \rightarrow q_{2}
\end{aligned}
$$


единственна и удовлетворяет уравнениям вида

$$
\left(\frac{\partial}{\partial t_{m}^{\prime}}-A_{m}^{\prime}\right) \psi=\left(\frac{\partial}{\partial t_{n}^{\prime \prime}}-A_{n}^{\prime \prime}\right) \psi=0
$$

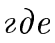

$$
A_{m}^{\prime}=\partial^{2 m+1}+a_{2 m-1}^{\prime} \partial^{2 m-1}+\cdots, \quad A_{n}^{\prime \prime}=\bar{\partial}^{2 n+1}+a_{2 n-1}^{\prime \prime} \bar{\partial}^{2 n-1}+\cdots
$$

Потенциаль $V\left(z, \bar{z}, t^{\prime}, t^{\prime \prime}\right)$, восстановленные по формуле (180), удовлетворяют нелинейным уравнениям, представимым $L, A, B$-тройками:

$$
\frac{\partial L}{\partial t_{m}^{\prime}}=\left[L, A_{m}^{\prime}\right]+B_{m}^{\prime} L, \quad \frac{\partial L}{\partial t_{n}^{\prime \prime}}=\left[L, A_{n}^{\prime \prime}\right]+B_{n}^{\prime \prime} L .
$$

Простейщие из них имеют вид

$$
\begin{aligned}
\frac{\partial V}{\partial t_{1}^{\prime}} & =\partial^{3} V+\partial(u V), & \bar{\partial} u & =3 \partial V \\
\frac{\partial V}{\partial t_{1}^{\prime \prime}} & =\bar{\partial}^{3} V+\bar{\partial}(w V), & \partial w & =3 \bar{\partial} V .
\end{aligned}
$$

Совместная деформаиия (187) и (188) по $t=t_{1}^{\prime}=t_{1}^{\prime \prime}$ :

$$
\frac{\partial V}{\partial t}=\partial^{3} V+\bar{\partial}^{3} V+\partial(u V)+\bar{\partial}(\bar{u} V)
$$

сохраняет класс вещественных потенциалов. Решения этих уравнений задаются формулами

$$
\begin{aligned}
& V\left(z, \bar{z}, t^{\prime}, t^{\prime \prime}\right) \\
& \quad=2 \partial \bar{\partial} \log \theta\left(U^{+} z+U^{-} \bar{z}+W_{1}^{+} t_{1}^{\prime}+\cdots+W_{1}^{-} t_{1}^{\prime \prime}+\cdots+Z(D), \operatorname{Pr}(\Gamma, \sigma)\right) .
\end{aligned}
$$

Вьвод формулы (190) аналогичен вьводу формулы (136).

Уравнение Веселова-Новикова (190) переходит в Кд $\Phi$, если потенциал $V$ не зависит от $y$, и является тем самым двумеризацией $\mathrm{Kд} \Phi$, отличной от КП.

11.2. Бесконечномерные алгебры Ли в теории солитонов и иерархии ВKР и DKP.

Иное определение иерархии КП (и ее обобщений), отличное от данного в $\S 10.5$, было предложено в серии работ Дате, Джимбо, Кашивары и Мивы, опиравшихся на идеи Сато [19]. Мы изложим его в форме, приближенной к обсуждавшимся выше методам.

Конечнозонные и солитонные решения уравнений КП имеют вид

$$
u=2 \partial_{x}^{2} \log \tau\left(x_{1}, x_{2}, \ldots\right) .
$$

В конечнозонном случае функция $\tau$ является, фактически, тэта-функцией (в физических переменных $\left.x=x_{1}, y=x_{2}, t=x_{3}\right)$ :

$$
\tau\left(x_{1}, x_{2}, \ldots\right)=\theta\left(U_{1} x_{1}+U_{2} x_{2}+\cdots+Z, \Omega\right),
$$


и решение всей иерархии $\tau$ принадлежит пространству формальных рядов $R=$ $\mathbb{C}\left[\left[x_{1}, x_{2}, \delta\right]\right]$ от бесконечного числа переменных.

Рассмотрим обшую ситуацию. Пусть група $G$ действует на векторном пространстве $V, v_{0} \in V$ и задан оператор $A$, коммутируюший с действием группы. Предположим, что $A\left(v_{0}\right)=0$. Тогда

$$
A\left(g \cdot v_{0}\right)=0 \text { для любого } g \in G \text {. }
$$

Рассмотрим групу $G=\mathrm{GL}(\infty)$, действуюшую диагонально на $V=R \otimes R$. Пусть $v_{0}=1 \otimes 1$ и оператор $A$ имеет вид

$$
\begin{aligned}
A \cdot \tau\left(x^{\prime}\right) \tau\left(x^{\prime \prime}\right)=\operatorname{Res}_{z=0}\left(\exp \sum_{j \geqslant 1}\right. & \left.z^{j}\left(x_{j}^{\prime}-x_{j}^{\prime \prime}\right)\right) \\
& \times \exp \left(-\sum_{j \geqslant 1} \frac{z^{j}}{j}\left(\frac{\partial}{\partial x_{j}^{\prime}}-\frac{\partial}{\partial x_{j}^{\prime \prime}}\right)\right) \cdot \tau\left(x^{\prime}\right) \tau\left(x^{\prime \prime}\right) .
\end{aligned}
$$

Оператор $A$ является примером вершинных операторов, открытых физиками в теории струн. На язьке квантовой теории поля данная система записьвается проще.

Пусть $F-\mathbb{Z}$-градуированное фермионное пространство Фока. На $F$ действует алгебра Клиффорда $\mathrm{Cl}$, порожденная образующими $\psi_{j}, \psi_{k}^{*}$, удовлетворяющими соотношениям $\psi_{j} \psi_{k}^{*}+\psi_{k}^{*} \psi_{j}=\delta_{j k}, \psi_{j}^{*} \psi_{k}^{*}+\psi_{k}^{*} \psi_{j}^{*}=\psi_{j} \psi_{k}+\psi_{k} \psi_{j}=0$. Определено вложение $\mathrm{gl}(\infty) \rightarrow \mathrm{Cl}: E_{j k} \rightarrow \psi_{j} \psi_{k}^{*}$ (где $E_{j k}$ - матрища с единственным ненулевым элементомединицей на пересечении $j$-й строки и $k$-го столбца). Оператор $A$ на $F \otimes F$ имеет вид $\sum_{j} \psi_{j} \otimes \psi_{j}^{*}$, а вектор $v_{0}=|0\rangle \otimes|0\rangle$. Легко проверить, что $A$ и действие алгебры $\mathrm{gl}(\infty)$ коммутируют. При бозонизации $F^{(0)}$ переходит в $R$, и мы получаем описанную вьше систему.

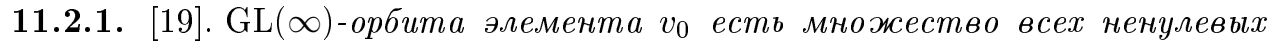
решений уравнения $A v=0$.

Подробное описание пространства Фока $F$ и бозонизации дано в [57].

Вьпишем систему (194) подробнее. Для этого введем переменные $x_{j}=\left(x_{j}^{\prime}+x_{j}^{\prime \prime}\right) / 2$, $y_{k}=\left(x_{j}^{\prime}-x_{j}^{\prime \prime}\right) / 2$, в которых $(194)$ примет вид

$$
\sum_{j \geqslant 0} S_{j}(2 y) S_{j+1}\left(-\widetilde{\partial}_{y}\right) \tau(x+y) \tau(x-y)=0
$$

где $\widetilde{\partial}_{y}=\left(\frac{\partial}{\partial y_{1}}, \frac{1}{2} \frac{\partial}{\partial y_{2}}, \frac{1}{3} \frac{\partial}{\partial y_{3}}, \ldots\right)$ и $S_{j}$ - элементарные полиномы Шура (см. (142)).

Уравнения (195) представимы в виде билинейных уравнений Xироты: если $P\left(x_{1}, x_{2}, \ldots\right)$ - полином от конечного числа переменных, то уравнением Хироты [50] называется уравнение вида

$$
\left.P\left(\frac{\partial}{\partial y_{1}}, \frac{\partial}{\partial y_{2}}, \ldots\right) f\left(x_{1}+y_{1}, \ldots\right) g\left(x_{1}-y_{1}, \ldots\right)\right|_{y=0}=0
$$


Разложим (195) в ряд Тейлора по $y_{1}, y_{2}, \ldots$ Каждый коэффициент этого разложения равен нулю, и это условие имеет вид уравнения Хироты. Например, равенство нулю коэффициента при $y_{3}$ влечет за собой

$$
\left(D_{1}^{4}-4 D_{1} D_{3}+3 D_{2}^{2}\right) \tau \cdot \tau=0
$$

Если в (197) перенормировать $x_{1}, x_{2}$ и $x_{3}$, заменить $\tau$ на $\theta$ и применить бинарную теорему сложения Римана, то мы получим уравнение эффективизации Дубровина для $\mathrm{K \Pi} \mathrm{(191)} \mathrm{при} d=0$.

Исходя из реализации представлений бесконечномерных алгебр Ли с помощю алгебр Клиффорда, Дате, Джимбо, Кашивара и Мива показали, как в этой ситуации возникает иерархия уравнений КдФ (она отвечает алгебре $A_{1}^{(1)}$ ), и вьвели новые иерархии - естественные аналоги КП.

В [19.IV] они ввели иерархию $B K P$, отвечающую центральному расширению алгебры so $(\infty)$ - алгебре $b_{\infty}$. Уравнения ВKР представимы уравнениями вида $(166)$ для

$$
B_{2 n+1}=\frac{\partial^{2 n+1}}{\partial x^{2 n+1}}+\sum_{k=1}^{2 n-1} b_{n k} \frac{\partial^{k}}{\partial x^{k}}, \quad B_{2 n} \equiv 0
$$

Наличие коммутационного представления позволило найти конечнозонные решения с помощью функций Бейкера-Ахиезера [19.V]. Они имеют вид (191)-(192) и выражаются через тэта-функции многообразий Прима двулистных накрытий с двумя точками ветвления, как и решения уравнений Веселова-Новикова. Начальные уравнения иерархии ВКР имеют вид (в форме Хироты)

$$
\begin{gathered}
\left(D_{1}^{6}-5 D_{1}^{3} D_{3}-5 D_{3}^{2}+9 D_{1} D_{5}\right) \tau \cdot \tau=0 \\
\left(D_{1}^{8}+7 D_{1}^{5} D_{3}-35 D_{1}^{2} D_{3}^{2}-21 D_{1}^{3} D_{5}-42 D_{3} D_{5}+90 D_{1} D_{7}\right) \tau \cdot \tau=0 .
\end{gathered}
$$

C алгеброй $\operatorname{sp}(\infty)$ связана иерархия CKP [19.VI], которая также допускает коммутационное представление. Ее конечнозонные решения строятся по двулистным накрытиям римановых поверхностей с $2 n>2$ точками ветвления. Многообразия Прима таких накрытий не главно поляризованы, и точные тэта-функциональные формулы для конечнозонных решений СКР до сих пор не вьписаны.

В [19.IV] были введены и $m$-компонентные иерархии ВKР, связанные с центральными расширениями алгебры so $(m \cdot \infty)$. Решения уравнений этих иерархий являются функциями от бесконечного числа переменных, разбитых на $m$ классов вида $\left(x_{1}^{(1)}, x_{2}^{(1)}, \ldots\right), \ldots,\left(x_{1}^{(m)}, x_{2}^{(m)}, \ldots\right)$. Простейшим примером является иерархия, отвечающая $m=2$. В этом случае первые нетривиальные уравнения имеют вид

$$
\begin{gathered}
\widehat{D}\left(D_{1}^{3}-D_{3}\right) \tau \cdot \tau=0, \\
\widehat{D}\left(D_{1}^{5}+5 D_{3} D_{1}^{2}-6 D_{5}\right) \tau \cdot \tau=0,
\end{gathered}
$$

где $D_{j}=D_{j}^{(1)}$ и $\widehat{D}=D_{1}^{(2)}$. В [19.VII] эта иерархия назьвается также BKP-II, а впоследствии ее стали именовать иерархией $D K P[56]$. Коммутационное представление для DKP не было найдено, и потому ее решения не обсуждались. 
Заметим, что иной подход к построению иерархий нелинейных уравнений, связанных с тэта-функциями Прима, рассматривался в [89].

Идеи работ [19] в дальнейшем интенсивно развивались в различных направлениях, из которых укажем два. Теорема 11.2.1, восходящая к Сато, имеет следующую простую трактовку: тау-функции иерархии КП параметризуются точками бесконечномерного грассманиана: $\mathbb{C}\left[\left[x_{1}, x_{2}, \ldots\right]\right] / \mathrm{GL}(\infty)$. Изучение клеточного разбиения этого грассманиана, связанного с разбиением всех возможных решений на различные классы (конечнозонные, рациональные, солитонные и др.), было предпринято в [97]. Другая интересная задача - о каноническом сопоставлении каждой группе петель иерархии солитонных уравнений - изучалась в [58].

\section{3. Уравнение Ландау-Лифшица.}

В [20] методы [19] были применены к известному в физике твердого тела уравнению Ландау-Лифшица (ЛЛ):

$$
S_{t}=\left[S \times S_{x x}\right]+[S \times J S]
$$

где $S$ - трехмерный вектор, $S_{1}^{2}+S_{2}^{2}+S_{3}^{2}=1$ и $J=\operatorname{diag}\left(J_{1}, J_{2}, J_{3}\right)$.

Его интегрируемость методами теории солитонов была установлена Боровиком и Скляниньм в конще 70-х годов. Уравнения Хироты для (201) были найдены в [51] на основе анзатца, предложенного физиками Богданом и Ковалевым:

$$
\begin{aligned}
& S_{1}=\left(f g^{*}+g f^{*}\right) /\left(f f^{*}+g g^{*}\right), \\
& S_{2}=-i\left(g f^{*}-f g^{*}\right) /\left(f f^{*}+g g^{*}\right), \\
& S_{3}=\left(f f^{*}-g g^{*}\right) /\left(f f^{*}+g g^{*}\right) .
\end{aligned}
$$

Они имеют вид

$$
\begin{gathered}
D_{1}\left(f \cdot f^{*}+g \cdot g^{*}\right)=0, \\
\left(D_{2}-D_{1}^{2}\right)\left(f \cdot f^{*}-g \cdot g^{*}\right)=0, \\
\left(D_{2}-D_{1}^{2}+\lambda\right) f \cdot g^{*}+\mu g \cdot f^{*}=0, \\
\left(D_{2}-D_{1}+\lambda\right) g \cdot f^{*}+\mu f \cdot g^{*}=0, \\
\lambda+\mu=J_{3}-J_{1}, \lambda-\mu=J_{3}-J_{2} .
\end{gathered}
$$

Как было указано в [20], конечнозонные решения уравнения ЛЛ выражаются через тэта-функции многообразий Прима неразветвленных накрытий одного весьма специального класса накрытий. Мы опишем его в $§ 12.3$.

\section{$\S$ 12. Методы конечнозонного интегрирования в теории отображения Прима}

\section{1. Аналоги теоремы Торелли для отображений Прима.}

Вьше мы рассматривали два отображения Прима, отвечаюших неразветвленным (116) и разветвленным в двух точках (99) двулистным накрытиям.

Аналоги теоремы Торелли для обоих из них в обшем случае неверны.

Для неразветвленных накрытий ситуация достаточно прояснена. 
12.1.1. 1) Отображсение Прима $\operatorname{Pr}: \mathscr{D} \mathscr{U}_{g} \rightarrow \mathscr{A}_{g-1}$ имеет в точке общего положения максимальный ранг. При $g \leqslant 5$ он равен $g(g-1) / 2<\operatorname{dim} \mathscr{D} \mathscr{U}_{g} u$ $\overline{\operatorname{Pr}(\mathscr{D} \mathscr{U})_{g}}=\mathscr{A}_{g-1}$, a npu $g \geqslant 6-3 g-3=\operatorname{dim} \mathscr{D} \mathcal{U}_{g}$.

2) (Донаги, Смит [25]). При $g=6$ отображение Прима является в точке общего положения 27-листным накрытием и $\overline{\operatorname{Pr}\left(\mathscr{D} \mathscr{U}_{6}\right)}=\mathscr{A}_{5}$.

3) (Канев [59], Фридман, Смит [38]). При $g \geqslant 7$ отображсение Прима инбективно в точке общего положения.

Тетрагональная конструкция Донаги показьвает, что при любом $g$ существуют главно поляризованные абелевы многообразия, являюшиеся многообразиями Прима нескольких неэквивалентных накрытий.

Случай накрытий, разветвленных в двух точках, почти не изучался. Солитонными методами было показано

12.1.2. [104]. Отображсение Прима $\operatorname{Pr:} \mathscr{D} \mathscr{R}_{g} \rightarrow \mathscr{A}_{g}$ имеет максимальный ранг в точке общего положения.

Это утверждение было доказано как вспомогательное при изучении аналога гипотезы Новикова в проблеме типа Римана-Шоттки (см. $§ 12.4)$.

12.2. Эффективизация тэта-функциональных формул для решений уравнения Веселова-Новикова.

Эффективизация тэта-функциональных формул для конечнозонных решений уравнения Веселова-Новикова была проведена в [104] (результаты были анонсированы автором в Докл. АН СССР, 1985, т. 285, с. 1067-1070) с помощью методов, развитых в $[27]$.

12.2.1. [104]. Пусть $\theta$ - тэта-функиия неразложсимого главно поляризованного абелева многообразия размерности $g$. Если формула

$$
\mathscr{U}_{Z}=2 \partial \bar{\partial} \log \theta(U z+V \bar{z}+W t+Z)+C
$$

задает при всех $Z \in \mathbb{C}^{g}$ решения “голоморфной” половинки уравнения Веселова-Новикова

$$
\begin{gathered}
\frac{\partial \mathscr{U}_{Z}}{\partial t}=\partial^{3} \mathscr{U}_{Z}+\partial\left(v^{\mathcal{U}_{Z}}\right), \\
v=6 \partial^{2} \log \theta(U z+V \bar{z}+W t+Z)+d,
\end{gathered}
$$

то выполняются соотношения

$$
\left(D_{2} D_{3}-4 D_{1}^{3} D_{2}-d D_{1} D_{2}-3 C D_{1}^{2}-a\right) \widehat{\theta}[n, 0]=0,
$$

где $a, d, C$ - постоянные, $D_{1}=\partial_{U}, D_{2}=\partial_{V}, D_{3}=\partial_{W}$. Для "антиголоморфной половинки" соотношения имеют тот же вид с заменой местами $U$ u $V$.

Заметим, что при $a=d=C=0$ уравнение (208) переходит в уравнение Хироты для первого уравнения иерархии DKP (200).

По любому двулистному разветвленному накрытию с двумя точками ветвления можно построить решение уравнения Веселова-Новикова. Отсюда следует, что для каждого многообразия Прима сушествуют такие векторы $U, V \in \mathbb{C}^{g} \backslash\{0\}, W \in \mathbb{C}^{g}$ и постоянные $a, d, C$, что соотношения (208) выполняются. Это привело Новикова к аналогу его гипотезы для многообразий Прима, которьй мы приведем сейчас, а обсудим в $\oint 12.4$. 
12.2.2. АНАЛОГ ГИПОТЕЗЫ НовИКОВА. Неразложимое главно поляризованное абелево многообразие является многообразием Прима двулистного накрытия с двумя точками ветвления тогда и только тогда, когда соотношения (208) разрешимьи (с $U, V \neq 0)$.

Соотношения (208) обладают следующим свойством.

12.2.3. [104]. Если абелево главно поляризованное многообразие неразложимо и векторы $U$ и $V$ линейно независимы, то по (208) вектор $W$ и постояннье $а$, $c, d$ восстанавливаются однозначно с точностью до преобразований

$$
\begin{aligned}
& U \rightarrow \lambda U, \quad V \rightarrow \mu V, \quad W \rightarrow \lambda^{3} W+\alpha U, \\
& a \rightarrow \lambda^{3} \mu a, \quad C \rightarrow \lambda \mu C, \quad d \rightarrow \lambda^{2} d+\alpha .
\end{aligned}
$$

В частности, при $g=2$ мы получаем прямую процедуру построения решений уравнения Веселова-Новикова по произвольному неразложимому главно поляризованному абелеву многообразию и произвольной паре линейно независимых векторов. Постоянная $C$ восстанавливается двумя различньми способами: по уравнениям эффективизации для “голоморфной” и “антиголоморфной” половинок уравнения Веселова-Новикова. Условие совпадения ответов влечет за собой нетривиальные соотношения на тэта-константы при $g=2$.

12.2.4. [103]. Пусть $\theta$ - тэта-функция от двух переменных и матрица $\left(a_{n}^{11} a_{n}^{12} a_{n}^{22} a_{n}\right)$ обратна матриче $\left(\widehat{\theta}_{11}[n, 0] \widehat{\theta}_{12}[n, 0] \widehat{\theta}_{22}[n, 0] \widehat{\theta}[n, 0]\right)$ :

$$
a_{n}^{p q} \widehat{\theta}_{k l}[n, 0]=\delta_{k}^{p} \delta_{l}^{q}, \quad a_{n} \widehat{\theta}_{k l}[n, 0]=a_{n}^{p q} \widehat{\theta}[n, 0]=0, \quad a_{n} \widehat{\theta}[n, 0]=1
$$

Тогда выполняются соотношения

$$
\begin{gathered}
\sum_{n}\left(a_{n}^{k m} \widehat{\theta}_{m m m m}[n, 0]+2 a_{n}^{k k} \widehat{\theta}_{k m m m}[n, 0]\right)=0 \\
\left.\sum_{n}\left(a_{n}^{k m} \widehat{\theta}_{k m m m}[n, 0]-a_{n}^{m m} \widehat{\theta}_{m m m m}[n, 0]\right)+3 a_{n}^{k k} \widehat{\theta}_{k k m m}[n, 0]\right)=0 \\
\sum_{n}\left(a_{n}^{11} \widehat{\theta}_{1112}[n, 0]-a_{n}^{22} \widehat{\theta}_{1222}[n, 0]\right)=0
\end{gathered}
$$

где $1 \leqslant k, m \leqslant 2, n \in \frac{1}{2}\left(\mathbb{Z}^{2} / 2 \mathbb{Z}^{2}\right)$.

12.3. Формулы четверных секущих и уравнения Веселова-Новикова, ВКР и Ландау-Лифшица.

Через $\vec{\theta}(z)$ будем обозначать вектор $\left(\theta\left[n_{1}, 0\right](z, 2 \Omega), \ldots, \theta\left[n_{r}, 0\right](z, 2 \Omega)\right)$, а через $u \wedge v$ - произведение векторов $u, v \in \mathbb{C}^{2^{g}}$ в алгебре Грассмана.

Пусть $\Gamma \rightarrow \Gamma / \sigma-$ двулистное накрытие с двумя точками ветвления $q_{1}, q_{2}$. Согласно формуле четверной секушей Фэя 6.3.1 для произвольной четверки точек $p_{1}, \ldots, p_{4} \in \Gamma$ вьполняется тождество

$$
\begin{aligned}
\vec{\theta}\left(p_{1}+p_{2}+p_{3}+p_{4}\right) \wedge \vec{\theta} & \left(p_{1}+p_{2}-p_{3}-p_{4}\right) \\
& \wedge \vec{\theta}\left(p_{1}+p_{3}-p_{2}-p_{4}\right) \wedge \vec{\theta}\left(p_{1}+p_{4}-p_{2}-p_{3}\right)=0
\end{aligned}
$$


где, как и в $\S 6$, под $p_{j}$ понимается $A_{\mathrm{Pr}}\left(p_{j}\right)$.

Введем локальные параметры $k_{j}^{-1}$ в окрестностях точек $q_{j}$ и рассмотрим разложение в ряд отображений Абеля-Прима:

$$
k_{1}^{-1} \rightarrow A_{\operatorname{Pr}}\left(q_{1}\right)+\sum_{m} \frac{U_{m}}{k_{1}^{m}}, \quad k_{2}^{-1} \rightarrow A_{\operatorname{Pr}}\left(q_{2}\right)+\sum_{n} \frac{V_{n}}{k_{2}^{n}}
$$

Если инволюция $\sigma$ обращает параметры, то $U_{2 m}=V_{2 n}=0$. Через $D_{j}$ обозначим дифференцирование в направлении вектора $U_{j}$, а через $\widehat{D}_{k}-$ в направлении $V_{k}$. Введем также оператор $T(k)$ :

$$
T\left(k_{1}^{-1}\right)=\exp \left(\sum_{m=1}^{\infty} \frac{D_{m}}{k^{m}}\right)=1+\sum_{n \geqslant 1} \frac{\Delta_{n}}{k_{1}^{n}} .
$$

Выберем в качестве начальной точки отображения Абеля-Прима точку $q_{1}$ : $A_{\operatorname{Pr}}\left(q_{1}\right)=0$. Так как $q_{2}-$ неподвижная точка инволюции $\sigma: \Gamma \rightarrow \Gamma$, то также можно считать, что $A_{\operatorname{Pr}}\left(q_{2}\right)=0$.

Рассмотрим вырождение (211), отвечающее случаю $p_{4}=q_{1}, p_{2}, p_{3}, p_{4} \rightarrow q_{1}$. Мы опустим детали, указав лиш окончательный ответ:

12.3.1. [105]. Уравнения

$$
\left.T\left(k_{1}^{-1}\right) \circ\left(\vec{\theta}(z) \wedge \Delta_{1} \vec{\theta}(z) \wedge \Delta_{1}^{2} \vec{\theta}(z) \wedge\left(\Delta_{1} \Delta_{2}-\Delta_{3}\right) \vec{\theta}(z)\right)\right|_{z=0} \equiv 0
$$

получаются из формуль четверной секущей Фәя (211) вырождением $p_{1}, p_{2}, p_{3}$, $p_{4} \rightarrow q_{1}$. Разлагая левую часть $(214)$ в ряд по $k_{1}^{-1}$, получим иерархию уравнений в форме Хиротьи, старшие члень которой, после возмохсной замены локального параметра, примут вид

$$
\left(\Delta_{n} \Delta_{1}-\Delta_{n-1} \Delta_{1}^{2}+\Delta_{n-2}\left(\Delta_{1} \Delta_{2}-\Delta_{3}\right)\right) \theta \cdot \theta=0
$$

Ее члень при $n=5,7$ совпадают с (199), и иерархия (214) совпадает с иерархией $B K P$.

12.3.2. [105]. Уравнения

$$
\left.T\left(k_{1}^{-1}\right) \circ\left(\vec{\theta}(z) \wedge D_{1} \vec{\theta}(z) \wedge \widehat{D}_{1} \vec{\theta}(z) \wedge D_{1} \widehat{D}_{1} \vec{\theta}(z)\right)\right|_{z=0} \equiv 0
$$

получаются из формуль четверной секущей Фәя (211) вьрождением $p_{1}, p_{2}, p_{3} \rightarrow$ $q_{1}, p_{4} \rightarrow q_{2}$. Разлагая левую часть $(216)$ в ряд по $k_{1}^{-1}$, получим иерархию уравнений в форме Хироты, старшие члень которой, после возмохсных замен локальных параметров, примут вид

$$
\left(\Delta_{n} \widehat{D}_{1}-\Delta_{n-1} \Delta_{1} \widehat{D}_{1}\right) \theta \cdot \theta=0 .
$$


Ее члень при $n=3,5$ имеют вид (200), и иерархия (216) совпадает с иерархией “голоморфных" половинок уравнений Веселова-Новикова или иерархией DKP.

Эти утверждения объясняют с точки зрения алгебраической геометрии существование двух иерархий нелинейных уравнений, связанных с разветвленными в двух точках двулистными накрытиями алгебраических кривых и близких по своим свойствам к иерархии Кадомцева-Петвиашвили.

Указанная эквивалентность части иерархии Веселова-Новикова и иерархии DKP объясняет и почему не существует коммутационного представления уравнений DKP c помощью скалярных дифференциальных операторов.

Мы не указываем явного вида конечнозонных решений уравнений ВКР. Заметим лишь, что из формул (212), (214), (215) они могут быть легко найдены.

Рассмотрим теперь тождество четверной секущей Бовилля-Дебарра 7.2.2. Мы применим его к классу неразветвленных накрытий, связанных с уравнением ЛандауЛифшица.

Пусть $\Gamma \rightarrow E$ - двулистное накрытие римановой поверхности рода 1 , разветвленное в $4 g$ точках, и множество точек ветвления инвариантно относительно сдвига на полупериод: $u \rightarrow u+\alpha$. Через $\sigma$ обозначим инволюцию $Г$, порожденную этим сдвигом, а через $\omega$ - инволюцию, переставляющую листы накрытия $\Gamma \rightarrow E$. Очевидно, что $\Gamma \rightarrow \Gamma / \sigma-$ неразветвленное двулистное накрытие и инволющии $\sigma$ и $\omega$ коммутируют: $\sigma \omega=\omega \sigma$. Назовем накрытие $\Gamma \rightarrow \Gamma / \sigma$ ЛЛ-накрытием.

Конечнозонные решения уравнения ЛЛ, как было впервые указано в [20], строятся по таким накрытиям. Явные формулы в тэта-функциях Прима были выведены в [10]. Выберем базис дифференциалов Прима $u_{k}$ так, что $\sigma^{*}\left(u_{k}\right)=\omega^{*}\left(u_{k}\right)=-u_{k}$. Зафиксируем точку $p_{0}-$ точку ветвления накрытия $\Gamma \rightarrow \Gamma / \omega-$ и введем вектор

$$
\mu_{k}=\int_{\sigma\left(p_{0}\right)}^{p_{0}} u_{k}
$$

Очевидно, что $2 \mu \equiv 0$ в $\operatorname{Pr}(\Gamma, \sigma)$. Тогда

$$
A_{\mathrm{Pr}}(p)=\frac{1}{2} \int_{\sigma(p)}^{p} u=\frac{1}{2}\left(\int_{p_{0}}^{p} u+\mu+\int_{\sigma(p)}^{\sigma\left(p_{0}\right)} u\right)=\int_{p_{0}}^{p} u+\frac{\mu}{2}
$$

Рассмотрим четверку точек $p_{1}, p_{2}, p_{3}, p_{4} \in \Gamma$ и обозначим через $\delta$ вектор

$$
\delta_{j}=\int_{\omega\left(p_{1}\right)}^{p_{1}} u_{j}
$$

Введем в окрестности точки $p=p_{1}$ локальньй параметр $\varepsilon$ и отображение АбеляПрима разложим в ряд по степеням $\varepsilon$ :

$$
A_{\operatorname{Pr}}(\varepsilon)=A_{\operatorname{Pr}}(p)+U_{1} \varepsilon+U_{2} \varepsilon^{2}+\cdots
$$

Через $D_{j}$ обозначим дифференцирование в направлении $U_{j}$.

Формула четверной секущей Бовилля-Дебарра имеет вид (211), где для краткости в аргументах тэта-функций мы обозначаем $A_{\operatorname{Pr}}(p)$ через $p$. 
Рассмотрим вырождение формулы четверной секущей при $p_{2}, p_{3} \rightarrow \sigma \omega(p)$, $p_{4} \rightarrow \omega(p)$. При этом на многообразии Прима

$$
\begin{gathered}
p_{2}, p_{3} \rightarrow p_{1}+\mu, \quad p_{4} \rightarrow-p_{1}-\mu, \\
\left(p_{1}+\cdots+p_{4}\right),\left(p_{1}+p_{2}-p_{3}-p_{4}\right),\left(p_{1}+p_{2}-p_{2}-p_{4}\right) \rightarrow \delta, \\
\left(p_{1}+p_{4}-p_{2}-p_{3}\right) \rightarrow-\delta-2 \mu .
\end{gathered}
$$

Простейшими соотношениями, вытекаюшими из вырождения формулы четверной секушей, будут

$$
\left(D_{2}-D_{1}^{2}+\alpha\right) \vec{\theta}(\delta)+\beta \vec{\theta}(2 \mu+\delta)=0 .
$$

Преобразуя (218) в уравнения Хироты с помощью бинарной теоремы сложения Римана, получаем (204) и (205) для $f=\theta(z+\mu+\delta), f^{*} \theta(z), g=-i \theta(z+\delta)$ и $g^{*}=-i \theta(z+\mu)$, где $\theta$ - тэта-функция Прима накрытия $\Gamma \bar{\Gamma} / \sigma((204)$ переходит в (205) при сдвиге аргумента $z \rightarrow z+\mu)$.

При таком выборе $f, f^{*}, g, g^{*}$ уравнение (202) эквивалентно

$$
D_{1}(\vec{\theta}(\mu+\delta)+\vec{\theta}(\mu-\delta))=0,
$$

а уравнение (203) - уравнению

$$
\left(D_{2}-D_{1}^{2}\right)(\vec{\theta}(\mu+\delta)-\vec{\theta}(\mu-\delta))=0,
$$

т.е. (202) и (203) являются тривиальньми следствиями симметричности функции $\vec{\theta}$ $(\vec{\theta}(z)=\vec{\theta}(-z))$. В итоге получаем

12.3.3. [106]. Уравнения иерархии ЛЛ получаются из формульи четверной секущей для ЛЛ-накрытий в пределе при $p_{2}, p_{3} \rightarrow \sigma \omega\left(p_{1}\right), p_{4} \rightarrow \omega\left(p_{1}\right)$.

Заметим, что класс ЛЛ-накрытий достаточно мал по сравнению со всеми неразветвленньми двулистными накрытиями. Действительно, ЛЛ-накрытия с $g$-мерным многообразием Прима задаются локально $2 g$ параметрами: один параметр задает конформный тип тора $E$ и $2 g-1$ параметр задают точки ветвления, взятые с точностью до сдвига на торе. Все накрытия с $g$-мерным многообразием Прима задаются $3 g\left(=\operatorname{dim} \mathscr{D}_{\mathcal{U}_{g+1}}\right)$ параметрами.

Отметим, что вырождения формул четверных секущих рассматривались в [47]. Возможные связи полученных в [47] результатов с солитонными уравнениями пока не обсуждались.

\section{4. Аналог проблемы Римана-Шоттки для многообразий Прима.}

У равнения Веселова-Новикова, ВКР и Ландау-Лифшшица могут быть рассмотрены в духе гипотезы Новикова как характеризуюшие абелевы многообразия, в чьих тэта-функциях они интегрируются. Особый интерес представляют первые две иерархии, чьи решения строятся по всем многообразиям Прима накрытий с двумя точками ветвления. В связи с этим вьше мы уже упоминали аналог гипотезы Новикова 12.2.2.

Аналог теоремы Дубровина 9.2.3 был получен в [103], [104]: 
12.4.1. Условие разрешимости соотношений (208) (уравнений эффективизации для конечнозонных решений уравнения Веселова-Новикова) выделяет в $\mathscr{A}_{g}$ подмногообразие, одна из компонент которого совпадает с $\left.\overline{\operatorname{Pr}\left(\mathscr{D} \mathscr{R}_{g}\right.}\right)$.

То есть уравнение Веселова-Новикова локально решает аналог проблемы Римана-Шоттки для многообразий Прима накрытий с двумя точками ветвления. С учетом 12.1.2 доказательство 12.4.1 сводится к вычислению размерности данного подмногообразия. При доказательствах теорем 9.2.3, 10.1.2 и 10.2.1, дающих локальные решения проблемы Римана-Шоттки, эту роль играла теорема Торелли.

Как оказалось, как использование высших уравнений иерархии Веселова-Новикова, так и иерархии ВКР для отбрасьвания других компонент встречает значительные трудности.

Во-первых, потенциальные двумерные операторы Шрёдингера, конечнозонные на нулевом уровне энергии, строятся и по особьм поверхностям:

1) пусть спектральная поверхность Г оператора Шрёдингера имеет также особенности - двойные точки $q_{1}, \ldots, q_{k}$;

2 ) все особые точки $q_{1}, \ldots, q_{n}$ являются неподвижными точками инволюции $\sigma$, которая не переставляет ветвей Г в их окрестностях [108].

Фактор-поверхность $\Gamma / \sigma$ имеет также $k$ двойных точек. Многообразия Якоби поверхностей Г и $Г / \sigma$ некомпактны, но многообразие Прима с точностью до изогении можно понимать как фактор-пространство $J(\Gamma) / J(\Gamma / \sigma)$, и оно компактно и главно поляризовано.

Во-вторых, при солитонном доказательстве теоремы 10.3.4 мы говорили о том, что КП-потоки порождают касательное пространство к многообразию Якоби поверхности Г. В случае иерархии ВКР это не так, и тэта-функциональное решение уравнений ВКР может быть сосредоточено на неполномерной обмотке главно поляризованного абелева многообразия.

Это объясняет следующую формулировку теоремы Шиоты [99], [100].

\subsection{2. Если функиия}

$$
\mathscr{U}_{Z}=2 \partial^{2} \log \theta\left(U x+V_{1} t_{1}+\cdots+Z, \Omega\right)
$$

при всех $Z \in \mathbb{C}^{g}$ задает решения иерархии уравнений ВКР и линейная оболочка векторов $U, V_{1}, \ldots$ совпадает $c \mathbb{C}^{g}$, то главно поляризованное абелево многообразие $\mathbb{C}^{g} /\left\{\mathbb{Z}^{g}+\Omega \mathbb{Z}^{g}\right\}$ является многообразием Прима двулистного накрытия $\Gamma \rightarrow \Gamma / \sigma$ с двумя неособыми точками ветвления и конечным числом двойных точек ветвления, в которых инволюиия $\sigma$ не переставляет ветви Г.

Аналогичный результат может быть получен и для иерархии Веселова-Новикова $[100]$.

С геометрической точки зрения аналог проблемы типа Римана-Шоттки для накрытий с двумя точками ветвления не обсуждался, в отличие от неразветвленных накрытий.

Наиболее близок по духу к солитонной теории следующий результат Бовилля и Дебарра [7] (аналог их же теоремы 10.6.2): 
12.4.3. Условие наличия четверной секущей вьделяет в $\mathscr{A}_{g}$ подмногообразие, одна из компонент которого совпадает с $\overline{\operatorname{Pr}\left(\mathscr{D} \mathscr{U}_{g+1}\right)}$ (семейством многообразий Прима двулистных неразветвленных накрытий).

Связь этого результата с теорией солитонных уравнений пока не ясна. По-видимому, уравнение Ландау-Лифшица может быть использовано, но для характеризации многообразий Прима ЛЛ-накрытий.

Вспоминая иные подходы к проблеме Римана-Шоттки, укажем на изучение Sing $\Theta$, проведенное в [83], [101]. Оно, в частности, позволило понять и описать различие многообразий Прима и Якоби. Рассматривались и иные геометрические подходы [94], на которых мы не будем останавливаться. Заметим лише, что и гипотеза Донаги о характере неоднозначности отображения Прима (см. обзор [102]), и аналог проблемы Римана-Шоттки для многообразий Прима до сих пор открыты.

\section{Заключительные замечания}

Мы укажем в заключение некоторые вопросы, которые остались за пределами этого обзора.

1. По-видимому, первым приложением теории солитонов к алгебраической геометрии явился следуюший результат Дубровина и Новикова [33]. Пусть $V_{g}$ - пространство модулей гиперэллиптических поверхностей рода $g$. Рассмотрим расслоение $M_{g} \rightarrow V_{g}$ со слоем $J(\Gamma), \Gamma \in V_{g}$. Над $M_{g}$ построим теперь $2 g+2$-листное накрытие $\widetilde{M}_{g}$, связанное с фиксацией одной из $2 g+2$ точек ветвления гиперэллиптического накрытия $\Gamma \rightarrow \mathbb{C P}^{1}$. Пространство $\widetilde{M}_{g}$ называется полныц м пространством модулей гиперәллиптических многообразий Якоби. В [33] было доказано, что многообразие $\widetilde{M}_{g}$ рационально.

Конечнозонная теория уравнений, связанных с гиперэллиптическими поверхностями, в дальнейшем привела к целому ряду замечательных результатов (см. их изложение в [82]).

2. Недавно Накаяшики [87], [88], развивавший идеи Сато, показал, что существует естественное определение интегрируемых нелинейных уравнений как условий коммутации матричных дифференциальных операторов. Они задают деформации векторных расслоений (Бейкера-Ахиезера) на произвольных (!) главно поляризованных абелевых многообразиях и потому не связаны с геометрией римановых поверхностей. Явные формулы для самих уравнений и их решений пока не вьписаны.

3. Тождество тройной секущей является далеким обобщением теоремы сложения для эллиптических функций (это обсуждается в [36]). Существуют и другие возможности обобщения этой теоремы - теоремы сложения для гиперэллиптических функций больших родов [4]. Их связь с солитонными уравнениями была замечена недавно и сейчас исследуется [11].

4. Категорным обобщениям соответствия Берчналла-Чонди-Кричевера посвящены работы Муласе [80] и Ли и Муласе [70], [71]. В [80] на категорном языке изложена часть работы Кричевера и Новикова [66], в которой иерархия КП реализовывалась как деформации оснащенных полустабильных векторных расслоений ранга $l \geqslant 1$ с классом Чжэня $c_{1}=l g$ над неособьми римановыми поверхностями рода $g$. Тэта-функщиональные тождества и формулы в [80], [70], [71] не обсуждаются. Пред- 
ставляется интересным связать с тэта-функциональньми формулами результаты работы [70], в которой многообразия Прима $n$-листных накрытий были неэффективно описаны с помошњю $n$-компонентных иерархий КП.

5. Мы совершенно не касались связи римановых поверхностей с конечномерными интегрируемыми системами, имеющими коммутационное представление (наиболее современньй обзор дан в [24]), и с разностньми солитонными уравнениями (обзор этих работ содержится в [30]).

\section{СПИСОК ЛИТЕРАТУРЫ}

[1] Andreotti A., Mayer A. L. On period relations for abelian integrals on algebraic curves // Ann. Scuola Norm. Super. Pisa. Ser. 3. 1967. V. 21. № 2. P. 189-238.

[2] Arbarello E., De Concini C. On a set of equations characterizing Riemann matrices // Ann. of Math. 1984. V. 120. P. 119-140.

[3] Arbarello E., De Concini C. Another proof of a conjecture of S. P. Novikov on periods of abelian integrals on Riemann surfaces // Duke Math. J. 1987. V. 54. P. 163-178.

[4] Baker H.F. Abel's theorem and the allied theory including the theory of theta functions. Cambridge: Cambridge Univ. Press, 1897.

[5] Beauville A. Prym varieties and the Schottky problem // Invent. Math. 1977. V. 41. P. 149-196.

[6] Beauville A., Debarre O. Une relation entre deux approches du problìne de Schottky // Invent. Math. 1986. V. 86. P. 195-207.

[7] Beauville A., Debarre O. Sur le problème de Schottky pour les variétés de Prym // Ann. Scuola Norm. Super. Pisa. Ser. IV. 1987. V. XIV. P. 613-623.

[8] Белоколос Е. Д., Бобенко А. И., Матвеев В. Б., Энольский В.З.Алгеброгеометрические принципы суперпозиции конечнозонных решений интегрируемых нелинейных уравнений // УМН. 1986. Т. 41. № 2. С. 3-42.

[9] Белоколос Е. Д., Энольский В. З. Обобщенньй анзатц Лэмба // ТМФ. 1982. Т. 53. № 2. C. 271-282.

[10] Бобенко А.И. Вещественные алгеброгеометрические решения уравнения ЛандауЛиффшица в тэта-функциях Прима // Функц. анализ и его прил. 1985. Т. 19. № 1. С. 6-19.

[11] Buchstaber V.M., Enolskii V.Z., Leykin D. V. Hyperelliptic Kleinian functions and applications // Proceedings of the Novikov seminar. Translations of the Amer. Math. Soc. (to appear).

[12] Бухштабер В. М., Кричевер И. М. Векторные теоремы сложения и функции Бейкера-Ахиезера // ТМФ. 1993. Т. 94. № 2. С. 202-212.

[13] Buchstaber V.M., Krichever I. M. Multi-dimensional vector addition theorems and the Riemann theta functions // International Mathematical Research Notes (to appear).

[14] Burchnall J. L., Chaundy T. W. Commutative ordinary differential operators // I.: Proc. London Math. Soc. 1922. V. 21. P. 420-440; // II.: Proc. Royal Soc. London (A). 1928. V. 118. P. 557-583; // III.: Proc. Royal Soc. London (A). 1931. V. 134. P. 471-485.

[15] Buser P., Sarnak P. On the period matrix of a Riemann surface of large genus (with an Appendix by J. H. Conway and N. J. A. Sloane) // Invent. Math. 1994. V. 117. P. 27-56.

[16] Чередник И.В. Об условиях вещественности в "конечнозонном" интегрировании // Докл. АН СССР. 1980. Т. 252. № 5. С. 1104-1107.

[17] Чжээн Шен-шэнь. Комплексные многообразия. М.: ИЛ, 1961.

[18] Клеменс Г. Мозаика теории комплексных кривых. М.: Мир, 1984.

[19] Date E., Jimbo M., Kashiwara M., Miwa T. Transformation groups for soliton equations // I.: Proc. Japan. Acad. 1981. V. 57A. P. 342-347; // II.: ibid.. P. 387-392; // III.: J. Phys. Soc. Japan. 1981. V. 50. P. 3806-3812; // IV.: Physica D. 1982. V. 4. P. 343-365; // V.: Publ. RIMS. 1982. V. 18. P. 1111-1119; // VI.: J. Phys. Soc. Japan. 1981. V. 50. P. 3813-3818; // VII.: Publ. RIMS. 1982. V. 18. P. 1077-1110. 
[20] Date E., Jimbo M., Kashiwara M., Miwa T. Landau-Lifshitz equation: solitons, quasi-periodic solutions and infinite dimensional Lie algebras // J. Phys. A. 1983. V. 16. P. 221-236.

[21] Debarre O. The trisecant conjecture for Pryms // Proceedings of Symposia in Pure Math. V. 49. Part 1. Providence, 1989. P. 621-626.

[22] Debarre O. Trisecant lines and Jacobians // J. Algebr. Geom. 1992. V. 1. P. 5-14.

[23] Donagi R. Big Schottky // Invent. Math. 1987. V. 89. P. 568-599.

[24] Donagi R., Markman E. Spectral curves, algebraically completely integrable Hamiltonian systems, and moduli of bundles // Lect. Notes in Math., alg-geom-9507017 (to appear).

[25] Donagi R., Smith R. The structure of the Prym map // Acta Math. 1981. V. 146. P. 25-102.

[26] Дринфельд В.Г.О коммутативных подкольцах некоторых некоммутативных колец // Функц. анализ и его прил. 1977. Т. 11. № 1. С. 11-14.

[27] Дубровин Б.А. Тэта-функции и нелинейные уравнения // УМН. 1981. Т. 36.№ 2. C. $11-80$.

[28] Дубровин Б. А. Уравнение Кадомцева-Петвиашвили и соотношения между матрицами периодов на римановых поверхностях // Изв. АН СССР. Сер. матем. 1981. Т. 45. C. $1015-1028$

[29] Дубровин Б. А., Кричевер И. М., Новиков С. П. Уравнение Шрёдингерав периодическом поле и римановы поверхности // Докл. АН СССР. 1976. Т. 229. № 1. С. 15-19.

[30] Дубровин Б. А., Кричевер И. М., Новиков С. П. Интегрируемые системы. І. // Современные проблемы математики. Фундаментальные направления. Т. 4. М.: ВИНИТИ, 1985. C. $179-285$.

[31] Дубровин Б. А., Матвеев В.Б., Новиков С. П. Нелинейные уравнения типа Кортевега-де Фриза, конечнозонные линейные операторы и абелевы многообразия // УМН. 1976. Т. 31. № 1. С. $55-136$.

[32] Дубровин Б.А., Натанзон С. М. Вещественные двухзонные решения уравнения sine-Gordon // Функц. анализ и его прил. 1982. Т. 16. № 1. С. 27-43.

[33] Дубровин Б.А., Новиков С.П. Периодическая задача для уравнений Кортевега-де Фриза и Штурма-Лиувилля. Их связь с алгебраической геометрией // Докл. АН CCCP. 1974. T. 219. №3. C. 531-534.

[34] Farkas H. Schottky-Jung theory // Proceedings of Symposia in Pure Math. V. 49. Part 1. Providence, 1989. P. 459-483.

[35] Farkas H., Rauch H. E. Period relations of Schottky type on Riemann surfaces // Ann. of Math. 1970. V. 92. P. 434-461.

[36] Fay J. D. Theta functions on Riemann surfaces // Lecture Notes in Math. V. 352. Berlin: Springer-Verlag, 1973.

[37] Fay J. D. Schottky relations on $\frac{1}{2}(C-C) / /$ Proceedings of Symposia in Pure Math. V. 49. Part 1. Providence, 1989. P. 485-502.

[38] Friedman R., Smith R. The generic Torelli theorem for the Prym map // Invent. Math. 1982. V. 64. P. 473-490.

[39] van Geemen B. Siegel modular forms vanishing on the moduli space of curves // Invent. Math. 1984. V. 78. P. 615-642.

[40] van Geemen B., van der Geer G. Kummer varieties and the moduli spaces of Abelian varieties // Amer. J. Math. 1986. V. 108. P. 615-641.

[41] Гриффитс Ф., Харрис Дж. Принципы алгебраической геометрии. М.: Мир, 1982.

[42] Гриневич П. Г. Рациональные решения уравнений коммутации дифференциальных операторов // Функц. анализ и его прил. 1982. Т. 16. №1. С. 19-24.

[43] Гриневич П.Г. Векторньй ранг коммутирующих матричных дифференциальных операторов. Доказательство критерия С.П. Новикова // Изв. АН СССР. Сер. матем. 1986. T. 50. № 3. C. $458-478$.

[44] Gunning R.C. On generalized theta functions // Amer. J. Math. 1981. V. 103. P. 411-435. 
[45] Gunning R. C. Some curves in Abelian varieties // Invent. Math. 1982. V. 66. P. 377-389.

[46] Gunning R. C. Riemann surfaces and their associated Wirtinger varieties // Bull. Amer. Math. Soc. 1984. V. 11. P. 287-316.

[47] Gunning R. C. Some identities for Abelian integrals // Amer. J. Math. 1986. V. 108. P. 39-74.

[48] Ганнинг Р., Росси Х. Аналитические функции многих комплексных переменных. М.: Мир, 1969.

[49] Хартсхорн Р. Алгебраическая геометрия. М.: Мир, 1981.

[50] Hirota R. Direct methods of finding exact solutions of nonlinear evolution equations. Bäcklund transformations, the inverse scattering method, solitons, and their applications // Lecture Notes in Math. V. 515. Berlin: Springer-Verlag, 1976. P. 40-68.

[51] Hirota R. Bilinearization of soliton equations // J. Phys. Soc. Japan. 1982. V. 51. P. $323-331$.

[52] Hoyt W. L. On products and algebraic families of Jacobian varieties // Ann. of Math. 1963. V. 77. P. 415-423.

[53] Igusa J. Theta-functions. Grundlehren der Math. Wiss., 194. Berlin: Springer-Verlag, 1972.

[54] Igusa J. On the irreducibility of Schottky divisor // J. Fac. Sci. Univ. Tokyo. 1981. V. 28. P. 531-545.

[55] Итс А.Р., Матвеев В.Б. Об операторах Хилла с конечным числом лакун // Функц. анализ и его прил. 1975. Т. 9. № 1. С. 69-70.

[56] Jimbo M., Miwa T. Solitons and infinite dimensional Lie algebras // Publ. RIMS. 1982. V. 19. P. $943-1001$.

[57] Кац В. Г. Бесконечномерные алгебры Ли. М.: Мир, 1993.

[58] Kac V.G., Wakimoto M. Exceptional hierarchies of soliton equations // Proceedings of Symposia in Pure Math. V. 49. Part 1. Providence, 1989. P. 191-237.

[59] Канев В. Глобальная теорема Торелли для многообразий Прима // Изв. АН СССР. Сер. матем. 1982. Т. 46. № 2. С. 244-268.

[60] Козел В.А., Котляров В.П. Почти периодические решения уравнения $u_{t t}-u_{x x}=$ $\sin u$ // Докл. АН УССР. Сер. А. 1976. № 10. С. 878-880.

[61] Кричевер И.М. Интегрирование нелинейных уравнений методами алгебраической геометрии // Функц. анализ и его прил. 1977. Т. 11. № 1. С. 15-31.

[62] Кричевер И.М. Методы алгебраической геометрии в теории нелинейных уравнений // УМН. 1977. Т. 32. №6. С. 183-208.

[63] Кричевер И.М. Коммутативные кольца обыкновенных линейных дифференциальных операторов // Функц. анализ и его прил. 1978. Т. 12. №3. С. 20-31.

[64] Кричевер И. М. Спектральная теория двумерных периодических операторов и ее приложения // УМН. 1989. Т. 44. № 2. С. 121-184.

[65] Кричевер И. М., Новиков С. П. Голоморфные расслоения над римановыми поверхностями и уравнение Кадомцева-Петвиашвили // Функц. анализ и его прил. 1978. Т. 12. № 4. C. 41-52.

[66] Кричевер И.М., Новиков С. П. Голоморфные расслоения над алгебраическими кривыми и нелинейные уравнения // УМН. 1980. Т. 35. №6. С. 47-68.

[67] Lax P.D. Integrals of nonlinear equations of evolution and solitary waves // Comm. Pure Appl. Math. 1968. V. 21. P. 467-490.

[68] Lax P. D. Periodic solutions of the KdV equations // Lect. in Appl. Math. 1974. V. 15. P. $85-96$.

[69] Lax P. D. Periodic solutions of the KdV equation // Comm. Rure Appl. Math. 1975. V. 28. P. $141-188$.

[70] Li Y., Mulase M. Category of morphisms of algebraic curves and a characterization of Prym varieties // Preprint MPI 92/24, Bonn, 1992.

[71] Li Y., Mulase M. Hitchin systems and KP equations // Intern. J. Math. 1996. V. 7. P. 227-244.

[72] Little J. Translation manifolds and the Schottky problem // Proceedings of Symposia in Pure Math. V. 49. Part 1. Providence, 1989. P. 517-529. 
[73] Манаков С. В. Метод обратной задачи рассеяния и двумерные эволюционные уравнения // УМН. 1976. Т. 31. № 5. С. 245-246.

[74] Марченко В. А. Периодическая задача Кортевега-де Фриса // Матем. сб. 1974. Т. 95. №3. C. 331-356.

[75] Matsusaka T. On a characterization of a jacobian variety // Mem. Coll. Sci. Kyoto. Ser. A. 1959. V. 32. P. 1-19.

[76] McKean H.P., van Moerbeke P. The spectrum of Hill's equation // Invent. Math. 1975. V. 30. P. 217-274.

[77] Мохов О.И. Коммутирующие обькновенные дифференциальные операторы ранга 3, отвечающие эллиптической кривой // УМН. 1982. Т. 37. № 4. С. 169-170.

[78] Мохов О.И. Коммутирующие дифференциальные операторы ранга 3 и нелинейные уравнения // Изв. АН СССР. Сер. матем. 1989. Т. 53. № 6. С. 1291-1315.

[79] Mulase M. Cohomological structure in soliton equations and Jacobian varieties // J. Diff. Geometry. 1984. V. 19. P. 403-430.

[80] Mulase M. Category of vector bundles on algebraic curves and infinite dimensional Grassmanians // Intern. J. Math. 1990. V. 1. P. 293-342.

[81] Mumford D. Abelian Varieties // Tata Studies in Math. Oxford: Oxford Univ. Press, 1970.

[82] Мамфорд Д. Лекции о тэта-функциях. М.: Мир, 1988.

[83] Mumford D. Prym varieties // Contributions to analysis. A collection of papers dedicated to L. Bers. New York: Acad. Press, 1974. P. 325-350.

[84] Mumford D. An algebro-geometric construction of commuting operators and of solutions to the Toda lattice equation, Korteweg de Vries equation and related non-linear equations // Int. Symp. On Algebraic Geometry (Kyoto, 1977). Tokyo: Kinokunija Book Store, 1978. P. $115-153$.

[85] Mumford D., Fogarty J., Kirwan F. Geometric Invariant Theory // Ergebnisse der Math. und ihrer Grenzbegiete, 34. Berlin: Springer-Verlag, 1994.

[86] Munos Porras J. M. Geometric characterization of Jacobians and the Schottky equations // Proceedings of Symposia in Pure Math. V. 49. Part 1. Providence, 1989. P. 541-552.

[87] Nakayashiki A. Structure of Baker-Akhiezer modules of principally polarized Abelian varieties, commuting partial differential operators and associated integrable systems // Duke Math. J. 1991. V. 62. P. 315-358.

[88] Nakayashiki A. Commuting partial differential operators and vector bundles over Abelian varieties // Amer. J. Math. 1994. V. 116. P. 65-100.

[89] Натанзон С. М. Дифференциальные уравнения на тэта-функции Прима. Критерий вещественности двумерных конечнозонных потенциалных операторов Шрёдингера // Функц. анализ и его прил. 1992. Т. 26. № 1. С. 17-26.

[90] Новиков С. П. Периодическая задача Кортевега-де Фриза // Функц. анализ и его прил. 1974. T. 8. №3. C. 54-66.

[91] Новиков С. П. Двумерные операторы Шрёдингера в периодических полях // Итоги науки и техники. Современные проблемы математики. Т. 23. М.: ВИНИТИ, 1983. С. 3-32.

[92] Новиков С. П., Гриневич П. Г. О спектральной теории коммутирующих операторов ранга 2 с периодическими коэффициентами // Функц. анализ и его прил. 1982. Т. 16. № 1. C. $25-26$.

[93] Previato E., Wilson G. Vector bundles over curves and solutions of the KP equation // Proceedings of Symposia in Pure Math. V. 49. Part 1. Providence, 1989. P. 553-569.

[94] Радионов Д. Ю. К проблемам Торелли и Шоттки для многообразий Прима // Изв. РАН. Сер. матем. 1994. Т. 58. № 5. С. 68-84.

[95] Sasaki R. Modular forms vanishing at the reducible points of the Siegel upperhalf space // J. Reine Angew. Math. 1983. V. 345. P. 111-121.

[96] Schottky F., Jung H. Neue Sätze über Symmetralfunktionen und die Abel'schen Funktionen der Riemann'schen Theorie // Akad. Wiss. Berlin, Phys. Math. Kl. 1909. P. 282-297.

[97] Segal G., Wilson G. Loop groups and equations of KdV type // Publ. Math. IHES. 1985. V. 61. P. 5-65. 
[98] Shiota T. Characterization of Jacobian varieties in terms of soliton equations // Invent. Math. 1986. V. 83. P. 333-382.

[99] Shiota T. Prym varieties and soliton equations // Infinite-dimensional Lie algebras and groups (Luminy-Marseille, 1988), Adv. Ser. Math. Phys., 7. Teaneck: World Sci. Publishing, 1989. P. 407-448.

[100] Shiota T. The KP equation and the Schottky problem // Sugaku expositions. 1990. V. 3. P. 183-211.

[101] Шокуров В. В. Многообразия Прима: теория и приложения // Изв. АН СССР. Сер. матем. 1984. Т. 47. № 4. С. $785-856$.

[102] Шокуров В.В.Алгебраические кривые и якобианы // Современные проблемы математики. Фундаментальные направления. Т. 36. М.: ВИНИТИ, 1989. С. 233-273.

[103] Тайманов И. А. Об аналоге гипотезы Новикова в проблеме типа Римана-Шоттки для многообразий Прима // Докл. АН СССР. 1987. Т. 293. № 5. С. 1065-1068.

[104] Тайманов И. А. Многообразия Прима разветвленных накрытий и нелинейные уравнения // Матем. сб. 1990. Т. 181. № 7. С. 934-950.

[105] Тайманов И. А. Тэта-функции Прима и иерархии нелинейных уравнений // Матем. заметки. 1991. Т. 50. №1. С. 98-107.

[106] Тайманов И. А. Уравнение Ландау-Лифшшица и четверные секущие многообразий Прима // Функц. анализ и его прил. 1993. Т. 27. № 3. С. 90-92.

[107] С. П. Новиков (ред. ) Теория солитонов. М.: Наука, 1980.

[108] Веселов А. П., Новиков С. П. Конечнозонные двумерные операторы Шрёдингера. Потенциальные операторы // Докл. АН СССР. 1984. Т. 279. № 4. С. 784-788.

[109] Веселов А. П., Новиков С. П. Конечнозонные двумерные потенциальные операторы Шрёдингера. Явные формулы и эволюционные уравнения // Докл. АН СССР. 1984. Т. 279. № 1. C. $20-24$.

[110] Веселов А.П., Шабат А. Б. Одевающая цепочка и спектральная теория оператора Шрёдингера // Функц. анализ и его прил. 1993. Т. 27. № 2. С. 1-21.

[111] Weil A. Zum Beweis des Torellischen Satzes // Nachrichten der Akademie der Wissenschaften in Göttingen. Mathemathisch-Physikalische Klasse. 1957. № 2. P. 33-53.

[112] Welters G.E. On flexes of the Kummer variety // Indagationes Math. 1983. V. 45. P. $501-520$.

[113] Welters G. E. A characterization of non-hyperelliptic Jacobi varieties // Invent. Math. 1983. V. 74. P. 437-440.

[114] Welters G. E. A criterion for Jacobi varieties // Ann. of Math. 1984. V. 120. P. 497-504.

Институт математики СО РАН

Поступила в редакцию 26.05.1996 